INSTITUT NATIONAL DE RECHERCHE EN INFORMATIQUE ET EN AUTOMATIQUE

\title{
A quantization tree method for pricing and hedging multi-dimensional American options
}

\author{
Vlad BALLY — Gilles PAGÈS — Jacques PRINTEMS
}

$N^{\circ} 4465$

Mai 2002

THÈME 4 



\title{
A quantization tree method for pricing and hedging multi-dimensional American options
}

\author{
Vlad BALLY* ${ }^{*}$, Gilles PAGÈS ${ }^{\dagger}$, Jacques PRINTEMS ${ }^{\ddagger}$ \\ Thème 4 - Simulation et optimisation \\ de systèmes complexes \\ Projet Mathfi \\ Rapport de recherche $\mathrm{n}^{\circ} 4465$ - Mai 2002 - 63 pages
}

\begin{abstract}
We present here the quantization method which is well-adapted for the pricing and hedging of American options on a basket of assets. Its purpose is to compute a large number of conditional expectations by projection of the diffusion on optimal grid designed to minimize the (square mean) projection error ([25]). An algorithm to computes such grids is described. We provide results concerning the orders of the approximation with respect to the regularity of the pay-off function and the global size of the grids. Numerical tests are performed in dimensions 2, 4, 6, 10 with American style exchange options. They show that our theoretical orders are probably pessimistic.
\end{abstract}

Key-words: American option pricing, Optimal Stopping, Snell envelope, quantization of random variables

\footnotetext{
* Univ. du Maine et projet MATHFI, INRIA. E-mail: bally@ccr.jussieu.fr

$\dagger$ Univ. Paris 6. E-mail: gpa@ccr.jussieu.fr.

$\ddagger$ Univ. Paris 12. E-mail: printems@univ-paris12.fr.
} 


\section{Une méthode d'arbre par quantification pour l'évaluation et la couverture d'options américaines sur un panier d'actifs}

Résumé : Nous proposons une méthode de quantification adaptée à l'évaluation et à la couverture d'options américaines sur des paniers d'actifs financiers. Elle repose sur le calcul d'un grand nombre d'espérances conditionnelles par projection de trajectoires de la diffusion sur des grilles optimalement disposées pour minimiser l'erreur (quadratique moyenne) ainsi commise ([25]). Un procédé algorithmique pour construire de telles grilles est explicité. Nous fournissons des résultats sur l'ordre d'approximation en fonction de la régularité de l'actif contingent (pay-off) et de la taille globale des grilles. Des tests numériques sont réalisés en dimensions 2, 4, 6, 10 avec des options américaines d'échange. Ces tests montrent que les estimations d'erreur théoriques sont vraissemblablement pessimistes.

Mots-clés : Évaluation d'options américaine, arrêt optimal, enveloppe de Snell, quantification de vecteurs aléatoires 


\section{Introduction}

The aim of this paper is to present, to study and to test a probabilistic method for pricing and hedging American style options on multidimensional baskets of traded assets. The asset dynamics follow a $d$-dimensional diffusion model between time 0 and a maturity time $T$. We especially focused on a classical extension of the Black \& Scholes model in which the volatility may depend on the asset prices. However, a large part of the algorithmic aspects of this paper can be applied to more general models.

Pricing an American option in a continuous time Markov process $\left(S_{t}\right)_{t \in[0, T]}$ consists in solving the continuous time optimal stopping problem related to an obstacle process. In this paper we are interested in "Markovian" obstacles of the form $h_{t}=\left(h\left(t, S_{t}\right)\right.$ which are the most commonly considered on financial markets. Roughly speaking, there are two types of numerical methods for this purpose:

- First, some purely deterministic approaches coming from Numerical Analysis: the solution of the optimal stopping problem admits a representation $v\left(t, S_{t}\right)$ where $v$ satisfies a parabolic variational inequality. So, the various discretizing techniques like finite difference or finite element methods yield an approximation of the function $v$ at discrete points of a regular time-space grid (see e.g. [32] for an application to a vanilla put option or [9] for a more comprehensive study).

- Secondly, some probabilistic methods based on the dynamic programming formula or on the approximation of the (lowest) optimal stopping time. In 1-dimension, the most popular approach to American option pricing and hedging remains the implementation of the dynamic programming formula on a Binomial tree, originally initiated by Cox-Ross \& Rubinstein as an elementary alternative to continuous time Black \& Scholes model. However, let us mention before the massive development of Mathematical Finance, the pioneering work by Kushner in 1977 (see [28] and also [29]) in which the Markov chain approximation was first introduced, including its links with the finite difference method.

These methods are quite efficient to handle vanilla American options on a single asset but they quickly become intractable as the number of the underlying assets increases. Usually, numerical methods become inefficient because the space grids are built regardless of the distributions of the asset prices. The same problem occurs for finite state Markov chain approximation "à la Kushner". For the the extension of binomial treed into multinomial trees, the difficulty comes from the geometric shape of a tree compatible with all the dimension and correlation constraints.

In the past recent years, the problem gave birth to an extensive literature in order to overcome the dimensionality problem. All of them finally lead to some finite state dynamic programming algorithm either in its direct form or through the backward approximation of the (lowest) optimal stopping time. In [8], Barraquant \& Martineau a sub-optimal 1dimensional problem is solved: it amounts to process as if the obstacle process itself had the Markov property. In [36], the algorithm devised by Longstaff \& Schwartz is based on conditional expectation approximation by regression, using a finite class $\varphi_{i}\left(S_{t}\right)$ of random variables derived from a "root" family $\left(\varphi_{i}\right)$. In [40], Tsitsiklis \& Van Roy use a similar idea but for a modified Markov transition. In [11], Braodie \& Glassermann generates some 
random grids at each time step and compute some companion weights using some statistical ideas based on the importance sampling theorem.

Finally in [22] and [23] Fournié et al. initiated a new approach, based on Malliavin calculus, to the computation of conditional expectations and of their derivatives with respect to a parameter. This leads to a pure Monte Carlo method. Lions and Régnier in [36] use the same approach to price and to compute Greeks for American options.

In this paper, we propose a probabilistic method based on grids like in the original finite state Markov chain approximation method. First we start as usual by a time discretization of the asset price process at times $t_{k}:=k T / n, k=0, \ldots, n$ and, if necessary, we introduce the Euler scheme of the diffusion price process (still denoted $S_{t_{k}}$ for a while). But we will not choose these grid a priori. We will use our ability to simulate large samples of the asset price diffusion process - or at least its Euler scheme - to produce at each discretization time $t_{k}$ a grid $\Gamma_{k}^{*}$ with a given size $N_{k}$ having the following property: the closest neighbour rule projection $\pi^{\Gamma^{*}}\left(S_{t_{k}}\right)$ of $S_{t_{k}}$ onto the grid $\Gamma_{k}^{*}$ is the the best least square approximation of $S_{t_{k}}$ among all random vectors $Z$ such that $|Z(\Omega)|=N$. Namely

$$
\left\|S_{t_{k}}-\pi^{\Gamma_{k}^{*}}\left(\widehat{S}_{t_{k}}\right)\right\|_{2}=\min \left\{\left\|S_{t_{k}}-Z\right\|_{2}\left|, Z: \Omega \rightarrow \mathbb{R}^{d},\right| Z(\Omega) \mid \leq N_{k}\right\} .
$$

In some sense we will produce and then use at each time step the best possible grid of size $N_{k}$ to approximate the $d$-dimensional random vector $S_{t_{k}}$. For historical reasons coming from Information Theory, $\pi^{\Gamma_{k}^{*}}\left(S_{t_{k}}\right)$ is often called the optimal quantizer of $S_{t_{k}}$. The resulting error bound $\left\|S_{t_{k}}-\pi^{\Gamma_{k}^{*}}\left(S_{t_{k}}\right)\right\|_{2}$ is called the lowest quadratic mean quantization error and has been extensively investigated in Signal Processing and Information Theory for more than 50 years ([26] or more recently [25]). Namely one knows that it goes to 0 as $N_{k}$ goes to infinity at a $O\left(N_{k}^{-\frac{1}{d}}\right)$ rate.

Except in some specific 1-dimensional cases of little numerical interest, no closed form is available for the grid $\Gamma_{k}^{*}$ that produces the optimal quantizer, nor for the induced lowest quantization error. In fact little is known on the geometric structure of optimal quantizer grids in higher dimension. However, starting from the integral representation

$$
\left\|S_{t_{k}}-\pi^{\Gamma}\left(\widehat{S}_{t_{k}}\right)\right\|_{2}^{2}=\mathbb{E}\left(\min _{x \in \Gamma}\left|S_{t_{k}}-x\right|^{2}\right)
$$

which is valid for any grid $\Gamma$ and using its regularity properties as an almost everywhere differentiable (symmetric) function of $\Gamma$, one may implement a stochastic gradient descent that converges to some - at least locally - optimal quantizer . Furthermore, the procedure yields as by-products the Voronoi companion parameters of the grid which are involved in the pricing of the American option (see below). Simulations (see section) confirm what was expected: the optimal quantizer grid gets concentrated at zones heavily weighted by the diffusion process.

At this stage the time discretization consists in approximating the original American option with payoff $\left(h\left(t, S_{t}\right)\right)_{0 l e t \leq T}$ by its so-called Bermuda counterpart to be exercised 
exclusively at discrete times $t_{k}, k=0, \ldots, n$. It is classical background that then the theoretical premium is the result of a backward dynamic programming formula. Our algorithm simply consists in replacing the random variables $S_{t_{k}}$ by their optimal quantizers $\widehat{S}_{t_{k}}:=\pi^{\Gamma_{k}^{*}}\left(S_{t_{k}}\right)$ and then to write down this backward procedure "in distribution". The weights that appears are known from the grid optimization procedure described above.

The second part of the paper is devoted to an extensive study of the rate of convergence of this algorithm a function of the accuracy of the time discretization $(T / n)$ and of the total number $N:=1+N_{1}+\cdots+N_{n}$ of elementary quantizers used to produce the successive optimal quantizer grids on each time layer. We propose an optimal procedure to dispatch a priori these $N \mathbb{R}^{d}$-valued vectors among the layers and we derive some error bounds depending on the mean quantization error when the payoff is Lipschitz continuous. When the quantizer of each layer is optimal we obtain an a priori error bounds of the form $C\left(n^{-1 / 2}+\right.$ $\left.n(N / n)^{-\frac{1}{d}}\right)$ which can be improved (1 instead of $1 / 2$ when the payoff is semi-convex).

Then we design an approximating quantized hedging strategy following the ideas by Follmer \& Sondermann on incomplete markets. We are in position to derive some error bounds, called local residual risks. To produce these error bounds, we combine some methods borrowed from Reflected Backward Stochastic Differential Equation Theory, analytical p.d.e. techniques and quantization theory. We get a global rate of convergence for the hedging strategy which seems to be the first of that kind.

The last part of the paper is devoted to the experimental validation of the method on multi-dimensional American exchange options on (geometric) index in a standard $d$ dimensional Black \& Scholes model.

A common fact of most probabilistic methods applied to the American option problem is in the approximation of the conditional expectations implied by the Snell envelope formulation of the problem (76). For example, in [36], the authors computed such conditional expectation by means of least-squares regression on a basis of well suited polynomial functions. Such method has the advantages of the regular approximation methods and the drawbacks of the global approximation methods. From a numerical point of view, our method is a grid method which relies on the approximation of the solution by piecewise constant functions. Its purpose is, as it is common to all probabilistic methods, to compute a large number of conditional expectations along the path of the associated diffusion process. The main differences with the scheme in [36] is that it is a local but irregular approximation scheme.

We will present numerical results which tend to show that when the grid are optimal (in the quadratic quantization sense), the spatial order of convergence is better than in usual grid methods. This rate, better than expected, makes up for the drawback of an irregular approximation. Two settings have been selected for simulation: "in-the-money" and "out-of-the-money", both in several dimensions $d=2,4,6,10$. The results show that computed prices are more accurately in the first setting (with a maximal error at most of $5 \%$ in 10-dimension). Such behaviour seems to be explained by a bigger effect of the numerical incompleteness of the market in the in-the-money setting. 
Among the advantages of the local feature of the quantization approximation method, a prominent one is that it naturally leads to higher order approximations of the price involving the space derivatives (the hedging). This is developed in [6]. A second asset is that when the diffusion process is a function of the Brownian motion like in the Black \& Scholes model, our method if completely parameter free and all computations can be performed once the optimal quantization of the Brownian motion has been achieved.

\section{The investigated model}

We consider a market on which are traded $d$ risky assets $S^{1}, \ldots, S^{d}$ and a deterministic riskless asset $S_{t}^{0}:=e^{r t}, r \in \mathbb{R}$ between time $t:=0$ and the maturity time $T>0$. One typical model for the price process of the risky assets is the following diffusion model

$$
d S_{t}^{i}=S_{t}^{i}\left(r d t+\sum_{j=1}^{q} \sigma_{i j}\left(e^{-r t} S_{t}\right) d W_{t}^{j}\right), S_{0}^{i}:=s_{0}^{i}>0,1 \leq i \leq d
$$

where $W:=\left(W^{1}, \ldots, W^{q}\right)$ is a standard $q$-dimensional Brownian Motion defined on a probability space $(\Omega, \mathcal{A}, \mathbb{P})$ and

$$
\sigma: \mathbb{R}^{d} \longrightarrow \mathcal{M}(d \times q) \text { is a bounded and Lipschitz continuous. }
$$

The filtration of interest will be the natural (completed) filtration $\underline{\mathcal{F}}:=\left(\mathcal{F}_{t}^{S}\right)_{t \in[0, T]}$ of $S$ (which coincides with that of the Brownian motion as soon as $\sigma \sigma^{*}(x)>0$ for every $x$ ).

For notational convenience, we will denote $c(x):=\operatorname{Diag}(x) \sigma(x)$. Note that $c$ and the drift $b(x):=r x$ are Lipschitz so that a unique strong solution exists for $(1)$ on $(\Omega, \mathcal{A}, \mathbb{P})$. Furthermore, it is classical background that, for every $p \geq 1$,

$$
\mathbb{E}_{s_{0}}\left(\sup _{t \in[0, T]}\left|S_{t}\right|^{p}\right)<C_{p}\left(1+\left|s_{0}\right|^{p}\right)
$$

The discounted price process $\widetilde{S}_{t}:=e^{-r t} S_{t}$ is then a positive $\mathbb{P}$-martingale satisfying

$$
d \widetilde{S}_{t}=c\left(\widetilde{S}_{t}\right) \cdot d W_{t}^{j}, \widetilde{S}_{0}:=s_{0} .
$$

$\mathbb{P}$ is the so-called risk neutral probability in Mathematical Finance terminology. As long as $q \neq d$, the usual completeness of the market necessarily fails. However, from numerical point of view, this has no influence on the implementation of the quantization method to compute the price of the derivatives: we just compute a $\mathbb{P}$-price. When coming to the problem of hedging these derivatives, then the completeness assumption becomes crucial and will lead us to assume that $q=d$ and that the diffusion coefficient $c(x)$ is invertible everywhere on $\left(\mathbb{R}_{+}^{*}\right)^{d}$. 
When $q=d$ and $\sigma(x) \equiv \sigma \in \mathcal{M}(d \times d),(1)$ is the usual $d$-dimensional Black \& Scholes model: the risky assets are geometric Brownian motions given by

$$
S_{t}^{i}=s_{0}^{i} \exp \left(\left(r-\frac{1}{2}\left|\sigma_{i .}\right|^{2}\right) t+\sum_{1 \leq j \leq d} \sigma_{i j} W_{t}^{j}\right), 1 \leq i \leq d .
$$

An American option related to a payoff process $\left(h_{t}\right)_{t \in[0, T]}$ is a contract that gives the right to receive once and only once the payoff $h_{t}$ at some time $t \in[0, T]$ where $\left(h_{t}\right)_{t \in[0, T]}$ is a $\underline{\mathcal{F}}$-adapted nonnegative process. In this paper we will always consider the sub-class of payoffs $h_{t}$ that only depends on $\left(t, S_{t}\right)$ i.e. satisfying

$$
h_{t}:=h\left(t, S_{t}\right), \quad t \in[0, T] \quad \text { where } h:[0, T] \longrightarrow \mathbb{R}_{+} \text {is a Lipschitz continuous. }
$$

Such payoffs are sometimes called vanilla payoffs. Under Assumptions (1) and (4), one has for every $p \geq 1$,

$$
\mathbb{E}\left(\sup _{t \in[0, T]}\left|h_{t}\right|^{p}\right)<+\infty
$$

One justifies that - in a complete market - the fair price $\mathcal{V}_{t}$ at time $t$ for such a contract satisfies

$$
\mathcal{V}_{t}:=e^{r t} \operatorname{ess} \sup \left\{\mathbb{E}\left(e^{-r \tau} h_{\tau} / \mathcal{F}_{t}\right), \tau \in \mathcal{T}_{t}\right\}
$$

where $\mathcal{T}_{t}:=\{\tau: \Omega \rightarrow[t, T], \underline{\mathcal{F}}$-stopping time $\}$. This simply means that the discounted price $\widetilde{\mathcal{V}}_{t}:=e^{-r t} \mathcal{V}_{t}$ of the option is the Snell envelope of the discounted American payoff

$$
\widetilde{h}_{t}:=\widetilde{h}\left(t, \widetilde{S}_{t}\right)
$$

with $\widetilde{h}(t, x)=e^{-r t} h\left(t, e^{r t} x\right)$. This result is based on a hedging argument on which we will come back later on. Note that $\sup _{t \in[0, T]}\left|\mathcal{V}_{t}\right| \leq \sup _{t \in[0, T]}\left|h_{t}\right| \in L^{p}, p \geq 1$.

One shows (see [9]) using the Markov property of the diffusion process $\left(S_{t}\right)_{t \in[0, T]}$ that $\mathcal{V}_{t}:=\nu\left(t, S_{t}\right)$ where $\nu$ solves the variational inequality

$$
\max \left(\frac{\partial \nu}{\partial t}+\mathcal{L}_{r, \sigma} \nu, \nu-h\right)=0, \quad \nu(T, .)=h(T, .) .
$$

where $\mathcal{L}_{r, \sigma}$ denotes the infinitesimal generator of the diffusion (1).

Then, it is clear that the approximation problem for $\mathcal{V}_{t}$ appears as special case of the approximate computation of the Snell envelope of a $d$-dimensional diffusion with Lipschitz coefficients. To solve this problem in 1-dimension, many methods are available. These methods can be classified in two families: the probabilistic ones based on a weak approximation of the diffusion process $\left(S_{t}\right)$ by a purely discrete dynamics (e.g. binomial trees, [?]) and the analytic ones based on numerical methods for solving the variational inequality (6) (e.g. finite difference or finite element methods). When the dimension $d$ of the market increases, these methods become inefficient. 
In [4] (see also [5]) is devised a new method based on quantization which can be implemented in higher dimension. Next section is devoted to a presentation of the method and of the theoretical a priori error bounds that have been already derived.

At this stage, one may assume without loss of generality that the interest rate $r$ in (1) is 0 since Equation (3) for $\widetilde{S}$ appears as a special case of (1) for $S$ since the function $\widetilde{h}(t, x)$ has the same regularity as $h$. (To derive the "true" formulae when $r \neq 0$ one just has to keep in mind the "original" equation $\left.d \widetilde{S}_{t}=c\left(S_{t}\right) \cdot d W_{t}\right)$.

\section{Pricing an American option using a quantization tree}

In that part, the specificity of the martingale diffusion dynamics proposed for the risky assets in (3) (with $r=0$ ) has little influence on the results, so it is costless to consider a general drifted Brownian diffusion

$$
d S_{t}=b\left(S_{t}\right) d t+c\left(S_{t}\right) \cdot d W_{t}, \quad S_{0}:=s_{0} \in \mathbb{R}^{d} .
$$

where $b: \mathbb{R}^{d} \rightarrow \mathbb{R}^{d}$ and $c:[0, T] \times \mathbb{R}^{d} \rightarrow \mathcal{M}(d \times q)$ are Lipschitz continuous vector fields and $\left(W_{t}\right)_{t \in[0, T]}$ is $q$-dimensional Brownian motion.

\subsection{Time discretization: the Bermuda options}

The exact simulation of a diffusion at time $t$ is usually out of reach (e.g. when $\sigma$ is not constant in the specified model (1)). So one uses a (Markovian) discretization scheme, easy to simulate, e.g. the Euler scheme:

$$
\bar{S}_{t_{k+1}}=\bar{S}_{t_{k}}+b\left(\bar{S}_{t_{k}}\right) \frac{T}{n}+c\left(\bar{S}_{t_{k}}\right) \cdot\left(W_{t_{k}}-W_{t_{k-1}}\right) .
$$

Then, the Snell envelope to be approximated by quantization is that of the Euler scheme.

Sometimes, the diffusion can be simulated simply, essentially because it appears as a closed form $S_{t}:=\varphi\left(t, W_{t}\right)$. This is the case of the regular multi-dimensional Black \& Scholes model (set $\sigma(x):=\sigma$ in (1)). Then, it is possible to consider directly the the Snell envelope of the homogeneous Markov chain $\left(S_{t_{k}}\right)_{0 \leq k \leq n}$ for quantization purpose.

This time discretization corresponds, in the derivative terminology, to approximating the original continuous time American option by a Bermuda option, either on $\bar{S}$ or on $S$ itself. By Bermuda option, one means that the set of possible exercise times is finite. Error bounds are available at these exercise times $t_{k}$ (see Theorem 1 below).

Whatsoever, we want to quantize the Snell envelope of a homogeneous discrete time Markov chain $\left(S_{t_{k}}\right.$ or $\left.\bar{S}_{t_{k}}\right)$ whose transition, denoted $P^{(n)}(x, d y)$, preserves Lipschitz continuity. More precisely, for every Lipschitz continuous $f: \mathbb{R}^{d} \rightarrow \mathbb{R}$

$$
\left[P^{(n)} f\right]_{L i p} \leq\left(1+C_{b, \sigma, T} \frac{T}{n}\right)[f]_{L i p} .
$$


(see, e.g., [4] for a proof). In fact this discrete time markovian setting is the natural framework for the method. Throughout this section, we will denote by the generic notation $\left(X_{k}\right)_{0 \leq k \leq n}$ any ( $L^{p}$-integrable) homogeneous $\mathcal{F}_{t_{k}}$-Markov chain whose transition $P^{(n)}$ satisfies (9) and we will denote by $\left(V_{k}\right)_{0 \leq k \leq n}$ its $\mathcal{F}_{t_{k}}$-Snell envelope of $h\left(t_{k}, X_{k}\right)$ defined by

$$
V_{k}:=\operatorname{ess} \sup \left\{\mathbb{E}\left(h\left(\theta, X_{\theta}\right) / \mathcal{F}_{t_{k}}\right), \theta \in \Theta_{k}\right\}
$$

where $\Theta_{k}$ denotes the set of $\left\{t_{k}, \ldots, t_{n}\right\}$-valued $\mathcal{F}_{t_{\ell}}$-stopping times. The Snell envelope $V_{k}$ satisfies the so-called backward dynamic programming formula (see [37]):

$$
\left\{\begin{array}{l}
V_{n}:=h\left(t_{n}, X_{n}\right), \\
V_{k}:=\max \left(h\left(t_{k}, X_{k}\right), \mathbb{E}\left(V_{k+1} / \mathcal{F}_{t_{k}}\right)\right), \quad 0 \leq k \leq n-1 .
\end{array}\right.
$$

One derives a dynamic programming formula in distribution: $V_{k}=v_{k}\left(X_{k}\right), k \in\{0, \ldots, n\}$, where the functions $v_{k}$ are recursively defined by

$$
\left\{\begin{array}{l}
v_{n}:=h\left(t_{n}, .\right), \\
v_{k}:=\max \left\{h\left(t_{k}, .\right), P^{(n)}\left(v_{k+1}\right)\right\}, 0 \leq k \leq n-1 .
\end{array}\right.
$$

This formula remains intractable for numerical computation since they require to compute at each time step a conditional expectation.

Theorem 1 below gives some $L^{p}$-error bounds that hold for $\mathcal{V}_{t_{k}}-V_{t_{k}}$ in our original diffusion framework. First we need to introduce some definition about the regularity of $h$.

Definition 1 A function $h: \mathbb{R}^{d} \longrightarrow \mathbb{R}$ is semi-convex if

$$
\forall x, y \in \mathbb{R}^{d}, \forall t \in \mathbb{R}_{+}, \quad h(t, y)-h(t, x) \geq\left(\delta_{h}(t, x) \mid y-x\right)-\rho|x-y|^{2}
$$

where $\delta_{h}$ is a bounded function on $\mathbb{R}_{+} \times \mathbb{R}^{d}$ and $\rho \geq 0$.

Remarks: Note that (12) appears as a convex assumption relaxed by $-\rho|x-y|^{2}$. In most situations, is used in the reverse sense i.e. $h(t, x)-h(t, y) \leq\left(\delta_{h}(t, x) \mid x-y\right)+\rho|x-y|^{2}$. The semi-convexity assumption is fulfilled by a wide class of functions:

- If $h(t,$.$) is \mathcal{C}^{1}$ for every $t \in \mathbb{R}_{+}$and $\frac{\partial h}{\partial x}(t, x)$ is $\rho$-Lipschitz in $x$, uniformly in $t$, then $h$ is semi-convex (with $\left.\delta_{h}(t, x):=\frac{\partial h}{\partial x}(t, x)\right)$.

- If $h(t,$.$) is convex for every t \in \mathbb{R}_{+}$with a derivative $\delta_{h}(t,$.$) (in the distribution$ sense) which is bounded in $(t, x)$, then $h$ is semi-convex (with $\rho=0$ ). Thus, it embodies most usual pay-off functions used for pricing vanilla and exotic American style options like $h(t, x):=e^{-r t}\left(K-\varphi\left(e^{r t} x\right)\right)_{+}$with $\varphi$ Lipschitz continuous (on sets $\{\varphi \leq L\}, L>0$ ).

The notion of semi-convex function seems to have been introduced in [18] for pricing onedimensional American options. See also [33] for recent developments in a similar setting. 
Theorem 1 (a) Let $h: \mathbb{R}^{d} \rightarrow \mathbb{R}$ be a Lipschitz continuous function and let $p \in[1,+\infty)$. Let $V_{n}$ denote the Snell envelope of $\left(\bar{S}_{t_{k}}\right)_{0 \leq k \leq n}$ or $\left(S_{t_{k}}\right)_{0 \leq k \leq n}$. There is some positive real constant $C$ depending on $[b]_{\text {Lip }},[c]_{\text {Lip }}$ and $p$ such that

$$
\forall n \in \mathbb{N}^{*}, \forall k \in\{0, \ldots, n\}, \quad\left\|\mathcal{V}_{t_{k}}-V_{k}\right\|_{p} \leq \frac{e^{C T}(1+|x|)}{\sqrt{n}} .
$$

(b) If $X_{k}=S_{t_{k}}, k=0, \ldots, n$ and if the obstacle $h$ is semi-convex, then

$$
\forall n \in \mathbb{N}^{*}, \forall k \in\{0, \ldots, n\}, \quad\left\|\mathcal{V}_{t_{k}}-V_{k}\right\|_{p} \leq \frac{e^{C T}(1+|x|)}{n}
$$

\subsection{Space discretization: the quantization tree}

\subsubsection{Abstract quantization}

The starting point of our method is simply to discretize the random variables $X_{k}$ of the Markov chain by introducing some random variables $\widehat{X}_{k}$ that can only take a finite number $N_{k}$ of values. Then, we want to approximate the Snell envelope $\left(V_{k}\right)_{0 \leq k \leq n}$ by a sequence $\left(\widehat{V}_{k}\right)_{0 \leq k \leq n}$ defined by a dynamic programming algorithm quite similar to (10) except that it involves:

- the random variables $\left(\widehat{X}_{k}\right)_{0 \leq k \leq n}$ instead of the $\left(X_{k}\right)_{0 \leq k \leq n}$ and

- the conditional expectation given $\widehat{X}_{k}$ instead of the regular conditional expectation given the past up to $k$, i.e. $\mathbb{E}_{k}:=\mathbb{E}\left(. / \mathcal{F}_{k}\right)$.

\subsubsection{Elementary abstract quantization, $L^{p}$-distortion}

Let $X$ be a $\mathbb{R}^{d}$-valued random vector defined on a probability space $(\Omega, \mathcal{A}, \mathbb{P})$. From a probabilistic point of view, $L^{p}$-quantization $(p \geq 1)$ consists in studying the best $L^{p}$-approximation of $X$ by random vectors $X^{\prime}$ taking at most $N$ fixed values $x_{1}, \cdots, x_{N} \in \mathbb{R}^{d}$. These random vectors read $X^{\prime}:=\sum_{i=1}^{N} x_{i} \mathbf{1}_{A_{i}}(X),\left(A_{i}\right)_{1 \leq i \leq N}$ Borel partition of $\mathbb{R}^{d}$. One easily proves that for a fixed $N$-tuple $x:=\left(x_{1}, \cdots, x_{N}\right) \in\left(\mathbb{R}^{d}\right)^{N}$, the $L^{p}$-mean error $\left\|X-X^{\prime}\right\|_{p}$ reaches its minimum at any Voronoi tessellation $\left(C_{i}(x)\right)_{1 \leq i \leq N}$ of $x$.

Definition 2 Let $x:=\left(x_{1}, \cdots, x_{N}\right) \in\left(\mathbb{R}^{d}\right)^{N}$. A partition $C_{1}(x), \ldots, C_{N}(x)$ of $\mathbb{R}^{d}$ is a Voronoi tessellation of the $N$-tuple $x$ if, for every $i \in\{1, \ldots, N\}, C_{i}(x)$ is a Borel set satisfying

$$
C_{i}(x) \subset\left\{y \in \mathbb{R}^{d} /\left|x_{i}-y\right|=\min _{1 \leq j \leq N}\left|y-x_{j}\right|\right\} .
$$

where $|$.$| (usually) denotes the canonical Euclidean norm.$

Note that, however the $i^{t h}$ tessel $C_{i}(x)$ always has the same closure and the same boundary, this boundary being included in at most $N-1$ hyperplanes. Furthermore if the distribution of $X$ weights no hyperplane, then the Voronoi tessellation is essentially unique so that all the " $x$-Voronoi quantizers" $\widehat{X}$ have the same distribution. 
The problem is then to estimate the $L^{p}$-mean quantization error $\|X-\widehat{X}\|_{p}$ between $X$ and a "Voronoi quantizer"

$$
\widehat{X}:=\sum_{i=1}^{N} x_{i} \mathbf{1}_{C_{i}(x)}(X) .
$$

Let $\mathbb{P}_{X}$ denote the distribution of $X$. Then, the $L^{p}$-mean quantization error is given by

$$
\|X-\widehat{X}\|_{p}^{p}=\sum_{i=1}^{N} \mathbb{E}\left(\mathbf{1}_{C_{i}(x)}\left|X-x_{i}\right|^{p}\right)=\mathbb{E}\left(\min _{1 \leq i \leq N}\left|X-x_{i}\right|^{p}\right)=\int_{\mathbb{R}^{d}} \min _{1 \leq i \leq N}\left|x_{i}-y\right|^{p} \mathbb{P}_{X}(d y) .
$$

The $L^{p}$-mean quantization error only depends upon the distribution $\mathbb{P}_{X}$ of $X$.

The next step is to specify the choice of the $N$-tuple $x:=\left(x_{1}, \ldots, x_{N}\right)$ so as to get the smallest possible $L^{p}$-mean quantization error and to evaluate how fast it goes to 0 as $N$ goes to infinity. This will be investigated further on in subsection 3.3.1, once the way we use quantization and it related error will have been developed.

\subsubsection{Quantization tree: a pseudo-Snell envelop}

We assume from now on that for every $k \in\{0,1, \ldots, n\}$, we have access some way or another to a Voronoi quantized random vector $\widehat{X}_{k}$ for $X_{k}$, using $N_{k}$ points $x_{1}^{k}, \cdots, x_{N_{k}}^{k}$.

The pseudo-dynamic programming formula below is devised by analogy with the original one (10): one simply replaces $X_{k}$ by its quantized random vector $\widehat{X}_{k}$ It reads as follows

$$
\left\{\begin{array}{l}
\widehat{V}_{n}:=h\left(t_{n}, \widehat{X}_{n}\right) \\
\widehat{V}_{k}:=\max \left(h\left(t_{k}, \widehat{X}_{k}\right), \mathbb{E}\left(\widehat{V}_{k+1} / \widehat{X}_{k}\right)\right), \quad 0 \leq k \leq n-1
\end{array}\right.
$$

Notation: for the sake of simplicity, from now on, we will denote $\widehat{\mathbb{E}}_{k}():.=\mathbb{E}\left(. / \widehat{X}_{k}\right)$.

The main reason for considering conditional expectation with respect to $\widehat{X}_{k}$ is that the the sequence $\left(\widehat{X}_{k}\right)_{k \in \mathbb{N}}$ is not Markovian. On the other hand, even if the $N_{k}$-tuple $x^{k}:=\left(x_{1}^{k}, \ldots, x_{N_{k}}^{k}\right)$ of every term $X_{k}$ of the chain has been set up a priori, this does not make possible to compute explicitly this algorithm. As a matter of fact, one needs to know the coupled distributions $\left(\widehat{X}_{k}, \widehat{X}_{k+1}\right), 0 \leq k \leq n-1$. This is enlightened by the easy Proposition below.

Proposition 1 Let $x^{k}:=\left(x_{1}^{k}, \ldots, x_{N_{k}}^{k}\right)$ denote for every $k \in\{0, \ldots, n\}$ a quantization of the distribution $\mathcal{L}\left(X_{k}\right)$. Set, for every $k \in\{0, \ldots, n\}$ and every $i \in\left\{1, \ldots, N_{k}\right\}$,

$$
\alpha_{i}^{k}:=\mathbb{P}\left(\widehat{X}_{k}=x_{i}^{k}\right)=\mathbb{P}\left(X_{k} \in C_{i}\left(x^{k}\right)\right)
$$


and, for every $k \in\{0, \ldots, n-1\}, i \in\left\{1, \ldots, N_{k}\right\}, j \in\left\{1, \ldots, N_{k+1}\right\}$

$$
\begin{aligned}
\pi_{i j}^{k} & :=\mathbb{P}\left(\widehat{X}_{k+1}=x_{j}^{k+1} / \widehat{X}_{k}=x_{i}^{k}\right)=\mathbb{P}\left(X_{k+1} \in C_{j}\left(x^{k+1}\right) / X_{k} \in C_{i}\left(x^{k}\right)\right) \\
& =\frac{\beta_{i j}^{k}}{\alpha_{i}^{k}} \quad \text { with } \quad \beta_{i j}^{k}:=\mathbb{P}\left(X_{k+1} \in C_{j}\left(x^{k+1}\right), X_{k} \in C_{i}\left(x^{k}\right)\right) .
\end{aligned}
$$

One defines by a backward induction the function $\widehat{v}_{k}$ by

$$
\begin{aligned}
& \widehat{v}_{n}\left(x_{i}^{n}\right):=h_{n}\left(x_{i}^{n}\right), \quad i \in\left\{0, \ldots, N_{n}\right\} \\
& \widehat{v}_{k}\left(x_{i}^{k}\right):=\max \left(h\left(t_{k}, x_{i}^{k}\right), \sum_{j=1}^{N_{k+1}} \pi_{i j}^{k} \widehat{v}_{k+1}\left(x_{j}^{k+1}\right)\right), 1 \leq i \leq N_{k}, 0 \leq k \leq, n-1 .
\end{aligned}
$$

Then, $\widehat{V}_{k}=\widehat{v}_{k}\left(\widehat{X}_{k}\right)$ satisfies the above dynamic programming (17) of the pseudo-Snell envelop. Thus, if $\mu_{0}:=\delta_{x_{0}}$, then $\widehat{v}_{0}\left(\widehat{X}_{0}\right)=\widehat{v}_{0}\left(x_{0}\right)$ is deterministic.

Simply implementing the algorithm defined by (20) on a computer raises two questions:

- How is it possible to estimate the parameters $\alpha_{i}^{k}$ and $\beta_{i j}^{k}$ involved in (20)?

- Is it possible to handle the complexity of such a tree structured algorithm?

Preliminary estimation Phase (FIrst apPROACH): the theoretical tractability of the above algorithm exclusively depends on the parameters $\alpha_{i}^{k}$ and $\beta_{i j}^{k}$. Actually, the ability to compute the $\alpha_{i}^{k}$ 's and the $\beta_{i j}^{k}$ 's at a reasonable cost is the key of the whole method presented here for practical implementation. The most elementary solution is simply to process a wide range regular Monte Carlo simulation of the Markov chain $\left(X_{k}\right)_{0 \leq k \leq n}$ to estimate the parameters $\alpha_{i}^{k}$ and $\beta_{i j}^{k}$ of interest defined by (18) and (19). An estimate of the $L^{p}$-mean quantization error $\left\|X_{k}-\widehat{X}_{k}\right\|_{p}$ can also be computed along the procedure. Actually, this ability to compute these weights and moduli at a reasonable cost is the key of the whole method. When $\left(X_{k}\right)_{0 \leq k \leq n}$ is a Euler scheme (or Black \& Scholes diffusion) this makes no problem. More generally, this depends upon the ability to simulate some $P(x, d y)$-distributed random numbers for any $x \in \mathbb{R}^{d}$.

We will see further on in paragraphs 3.3.1 and 3.3.2 how to choose the $N_{k}$-tuples $x^{k}$ (size and geometric location).

COMPLEXITY OF THE QUANTIZATION TREE : THEORY AND PRACTISE A quick look at the structure of the algorithm (20) shows that going from layer $k+1$ down to layer $k$ needs $C \times N_{k} \cdot N_{k+1}$ elementary computations ( $C$ is a positive real constant). Hence, the cost of a full tree descent in order to get $\left(\widehat{v}_{0}\left(x_{i}^{0}\right)\right)_{1 \leq i \leq N_{0}}$ approximately is

$$
\text { Complexity }=C \times\left(N_{0} N_{1}+N_{1} N_{2}+\cdots+N_{k} N_{k+1}+\cdots+N_{n-1} N_{n}\right) \text {. }
$$


Setting $N:=N_{0}+\cdots+N_{n}$ shows that this complexity is at most equal to

$$
\text { Complexity } \leq C \cdot \frac{N^{2}}{n+1} \quad \text { whatever the } N_{k} \text { 's are. }
$$

This "blind" combinatorial estimate needs to be tuned. In fact, in most examples the Markov transition $P(x, d y)$ behaves in such a way that, at each layer $k$, many terms of the "transition matrix" $\left[\pi_{i j}^{k}\right]$ are numerically 0 . This means that the estimates of these coefficients will often be 0! Subsequently, the true complexity of the algorithm is more likely close to $O(N)$ instead of the above $N^{2} / n$ estimation. Thus, the cost of such a "descent" is similar to that of a Cox-Ross-Rubinstein's one dimensional binomial tree with $O(\sqrt{N})$ time discretization instants (such a tree approximately contains $N / 2$ points).

\subsection{Convergence and rate}

The aim of this paragraph is to provide some a priori $L^{p}$-error bounds for $\left\|V_{k}-\widehat{V}_{k}\right\|_{p}, 0 \leq$ $k \leq n$, based on the $L^{p}$-mean quantization errors i.e. $\left\|X_{k}-\widehat{X}_{k}\right\|_{p}, 0 \leq k \leq n$ where quantizer $\widehat{X}_{k}$ is a Voronoi quantizer that takes $N_{k}$ values $x_{1}^{k}, \ldots, x_{N_{k}}^{k}$. This error modulus can be obtained as a by-product of a Monte Carlo simulation of $\left(X_{k}\right)_{0 \leq k \leq n}$ : it only requires to compute, for every $\mu_{k}$-distributed simulated random vector, its distance to its closest neighbour in the set $\left\{x_{1}^{k}, \ldots, x_{N_{k}}^{k}\right\}$.

The estimates below can be obtained for any Markov chain having a Lipschitz transition $P(x, d y)$ i.e. satisfying, for every Lipschitz continuous function $g: \mathbb{R}^{d} \longrightarrow \mathbb{R}$,

$$
[P g]_{L i p} \leq K[g]_{L i p} \quad \text { where } \quad[f]_{L i p}:=\sup _{x \neq y} \frac{|f(x)-f(y)|}{|x-y|} .
$$

This is the case of the Euler scheme (and the diffusion) having Lipschitz drift and diffusion coefficient as mentioned before see (9).

The theorem below specifies the Lipschitz regularity of the functions $u_{k}$ defined in (11) and gives the a priori error bounds in this Lipschitz setting.

Theorem 2 Assume that the function $h$ is $[h]_{L i p}$-Lipschitz continuous in $x$, uniformly time and that the transition $P$ is $K$-Lipschitz.

(a) The functions $u_{k}$ defined by (11) are Lipschitz continuous and

$$
\left[u_{k}\right]_{L i p} \leq(K \vee 1)^{n-k}[h]_{L i p} .
$$

(b) For every $k \in\{0, \ldots, n\}$, let $\widehat{X}_{k}$ denote a (Voronoi) quantizer of $X_{k}$. For every $p \geq 1$,

$$
\left\|V_{k}-\widehat{V}_{k}\right\|_{p} \leq \sum_{i=k}^{n} d_{i}\left\|X_{i}-\widehat{X}_{i}\right\|_{p}
$$

with $d_{i}:=[h]_{L i p}+c K\left[u_{i+1}\right]_{L i p}, 0 \leq i \leq n-1$ and $d_{n}:=[h]_{L i p}$. The real constant $c:=2$ if $p \neq 2$ and $c:=1$ if $p=2$. 
Proof: $(a)$ Clearly, $\left[u_{n}\right]_{L i p} \leq[h]_{L i p}$. Then, using that $\left|\max (a, b)-\max \left(a^{\prime}, b^{\prime}\right)\right| \leq \max (\mid a-$ $\left.a^{\prime}|| b-,b^{\prime} \mid\right)$, it follows from the dynamic programming equality (11) that

$$
\left[u_{k}\right]_{L i p} \leq \max \left([h]_{L i p},\left[P\left(u_{k+1}\right)\right]_{L i p}\right) \leq \max \left([h]_{L i p}, K\left[u_{k+1}\right]_{L i p}\right)
$$

One concludes by induction.

(b) Set $\Phi_{k}:=P\left(u_{k+1}\right)$ for every $k \in\{0, \ldots, n-1\}$ (and $\Phi_{n} \equiv 0$ ). The function $\Phi_{k}$ satisfies $\mathbb{E}\left(V_{k+1}\left(X_{k+1}\right)\right)=\Phi_{k}\left(X_{k}\right)$. One defines similarly $\widehat{\Phi}_{k}$ by the equality $\widehat{\mathbb{E}}_{k}\left(\widehat{V}_{k+1} / \widehat{X}_{k+1}\right):=$ $\widehat{\Phi}_{k}\left(\widehat{X}_{k}\right)\left(\right.$ and $\left.\widehat{\Phi}_{n} \equiv 0\right)$. Then

$$
\begin{aligned}
\left|V_{k}-\widehat{V}_{k}\right| \leq\left|h_{k}\left(X_{k}\right)-h_{k}\left(\widehat{X}_{k}\right)\right|+\left|\Phi_{k}\left(X_{k}\right)-\widehat{\Phi}_{k}\left(\widehat{X}_{k}\right)\right| & \\
\leq & {[h]_{L i p}\left|X_{k}-\widehat{X}_{k}\right|+\left|\Phi_{k}\left(X_{k}\right)-\widehat{\mathbb{E}}_{k}\left(\Phi_{k}\left(X_{k}\right)\right)\right|+\left|\widehat{\mathbb{E}}_{k}\left(\Phi_{k}\left(X_{k}\right)\right)-\widehat{\Phi}_{k}\left(\widehat{X}_{k}\right)\right| . } \\
\text { Now }\left|\Phi_{k}\left(X_{k}\right)-\widehat{\mathbb{E}}_{k} \Phi_{k}\left(X_{k}\right)\right| & \leq\left|\Phi_{k}\left(X_{k}\right)-\Phi_{k}\left(\widehat{X}_{k}\right)\right|+\widehat{\mathbb{E}}_{k}\left|\Phi_{k}\left(X_{k}\right)-\widehat{\mathbb{E}}_{k}\left(\Phi_{k}\left(\widehat{X}_{k}\right)\right)\right| \\
& \leq\left[\Phi_{k}\right]_{L i p}\left(\left|X_{k}-\widehat{X}_{k}\right|+\widehat{\mathbb{E}}_{k}\left|X_{k}-\widehat{X}_{k}\right|\right) .
\end{aligned}
$$

(Note that $\widehat{X}_{k}$ is $\mathcal{F}_{k}$-measurable.) Hence,

$$
\left\|\Phi_{k}\left(X_{k}\right)-\widehat{\mathbb{E}} \Phi_{k}\left(X_{k}\right)\right\|_{p} \leq c\left[\Phi_{k}\right]_{L i p}\left\|X_{k}-\widehat{X}_{k}\right\|_{p}
$$

with $c=2$. When $p=2$, the very definition of the conditional expectation as a projection in a Hilbert space implies that holds with $c=1$.

On the other hand, coming back to the definition of $\Phi_{k}\left(X_{k}\right)$ and $\widehat{\Phi}_{k}\left(\widehat{X}_{k}\right)$, one gets, using that $\widehat{\mathbb{E}}_{k} \circ \mathbb{E}_{k}=\widehat{\mathbb{E}}_{k}$,

$$
\left|\widehat{\mathbb{E}}_{k}\left(\Phi_{k}\left(X_{k}\right)\right)-\widehat{\Phi}_{k}\left(\widehat{X}_{k}\right)\right| \leq \widehat{\mathbb{E}}_{k}\left|V_{k+1}-\widehat{V}_{k+1}\right| .
$$

Any conditional expectation being a $L^{p}$-contraction, it follows that

$$
\left\|\widehat{\mathbb{E}}_{k}\left(\Phi_{k}\left(X_{k}\right)\right)-\widehat{\Phi}_{k}\left(\widehat{X}_{k}\right)\right\|_{p} \leq\left\|V_{k+1}-\widehat{V}_{k+1}\right\|_{p} .
$$

Finally, for every $k \in\{0, \ldots, n-1\}$,

$$
\begin{aligned}
\left\|V_{k}-\widehat{V}_{k}\right\|_{p} & \leq[h]_{L i p}\left\|X_{k}-\widehat{X}_{k}\right\|_{p}+\left\|\Phi_{k}\left(X_{k}\right)-\widehat{\mathbb{E}}_{k}\left(\Phi_{k}\left(X_{k}\right)\right)\right\|_{p}+\left\|\Phi_{k}\left(X_{k}\right)-\widehat{\Phi}_{k}\left(\widehat{X}_{k}\right)\right\|_{p} \\
& \leq\left\|V_{k+1}-\widehat{V}_{k+1}\right\|_{p}+\left([h]_{L i p}+c\left[\Phi_{k}\right]_{L i p}\right)\left\|X_{k}-\widehat{X}_{k}\right\|_{p} .
\end{aligned}
$$

INRIA 
On the other hand, $\left\|V_{n}-\widehat{V}_{n}\right\|_{p} \leq[h]_{L i p}\left\|X_{n}-\widehat{X}_{n}\right\|_{p}$, so that

$$
\left\|V_{k}-\widehat{V}_{k}\right\|_{p} \leq \sum_{i=k}^{n}\left([h]_{L i p}+c\left[\Phi_{i}\right]_{L i p}\right)\left\|X_{i}-\widehat{X}_{i}\right\|_{p}
$$

The definition of $\Phi_{i}$ and the $K$-Lipschitz property of the transition $P(x, d y)$ imply that

$$
\left[\Phi_{i}\right]_{L i p}=\left[P\left(u_{i+1}\right)\right]_{L i p} \leq K\left[u_{i+1}\right]_{L i p}
$$

and so $(a)$ is proved. $\diamond$

\subsubsection{Optimal quantization: existence and asymptotics}

The $L^{p}$-quantization error has a an attractive specificity among other usual error bounds used in Numerical Integration: it behaves as a regular function of the quantizing $N$-tuple $x:=\left(x^{1}, \ldots, x^{N}\right)$. More precisely, as a symmetric function of the $N$-tuple $x$, the $L^{p_{-}}$ quantization error is 1-Lipschitz continuous. If $\mathbb{P}_{X}$ has a compact support, it is straightforward that $x \mapsto\left\|X-\widehat{X}^{x}\right\|_{p}$ reaches a minimum at $x^{*}$. One may always assume that $x^{*} \in\left(\mathcal{H}\left(\operatorname{supp} \mathbb{P}_{X}\right)\right)^{N}\left(\right.$ convex hull of supp $\left.\mathbb{P}_{X}\right)$. When $\mathbb{P}_{X}$ no longer has a compact support, one shows by induction on $N$ that

$$
x \mapsto\left\|X-\widehat{X}^{x}\right\|_{p} \text { still reaches an absolute minimum on }\left(\mathbb{R}^{d}\right)^{N}
$$

(see [38] or [25], among others), still lying in $\left(\mathcal{H}\left(\operatorname{supp} \mathbb{P}_{X}\right)\right)^{N}$. Furthermore, one shows the following simple facts (see [38] or [25] and references therein):

- If $\operatorname{supp} \mathbb{P}_{X}$ has an infinite support, any optimal $N$-tuple $x^{*}$ has pairwise distinct elements.

- If $\operatorname{supp} \mathbb{P}_{X}$ is everywhere dense in its convex hull, then the $N$ components of an optimal $N$-tuple $x^{*}$ all lies in $\mathcal{H}(\operatorname{supp} \mu)$. This still holds true for $N$-tuples corresponding to local minima. In particular, this holds if $\mathbb{P}_{X}$ has a positive density function on $\mathbb{R}^{d}$.

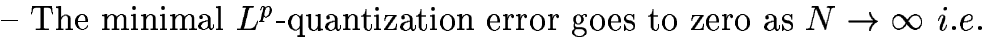

$$
\lim _{N} \min _{x \in\left(\mathbb{R}^{d}\right)^{N}}\left\|X-\widehat{X}^{x}\right\|_{p}=0
$$

As a matter of fact, let $\left(z_{k}\right)_{k \in \mathbb{N}}$ denote an everywhere dense sequence of $\mathbb{R}^{d}$-valued vectors and set $x_{N}:=\left\{z_{1}, \ldots, z_{N}\right\}$. It is straightforward that $\left\|X-\widehat{X}^{x_{N}}\right\|_{p}$ goes to zero by the Lebesgue Dominated Convergence Theorem. Furthermore $0 \leq \min _{x \in\left(\mathbb{R}^{d}\right)^{N}}\left\|X-\widehat{X}^{x}\right\|_{p} \leq$ $\left\|X-\widehat{X}^{x_{N}}\right\|_{p} \cdot \diamond$

At which rate does this convergence to zero hold turns out to be a much more challenging question. The answer was completed by several authors (Zador, see [26], Bucklew \& Wise, see [12] and finally Graf \& Luschgy see [25]). It reads as follows 
Theorem 3 Assume that $E|X|^{p+\eta}<+\infty$ for some $\eta>0$. Then

$$
\lim _{N}\left(N^{\frac{p}{d}} \min _{x \in\left(\mathbb{R}^{d}\right)^{N}}\left\|X-\widehat{X}^{x}\right\|_{p}^{p}\right)=J_{p, d}\|\varphi\|_{\frac{d}{d+p}}
$$

where $\mathbb{P}_{X}(d u)=\varphi(u) \lambda_{d}(d u)+\nu(d u), \nu \perp \lambda_{d}\left(\lambda_{d}\right.$ Lebesgue measure on $\left.\mathbb{R}^{d}\right)$ and $\|g\|_{q}:=$ $\left(\int|g|^{q}(x) d x\right)^{\frac{1}{q}}$ for every $q \in \mathbb{R}_{+}^{*}$. The constant $J_{p, d}$ corresponds to the case of the uniform distribution on $[0,1]^{d}$ (or any Borel set of Lebesgue measure 1 ).

Little is known about the true value of the constant $J_{p, d}$ except in dimension 1 where $J_{p, 1}=\frac{1}{2^{p}(p+1)}$. Some geometric considerations lead to $J_{2,2}=\frac{5}{18 \sqrt{3}}$ (see [26]). Nevertheless (see [?] or [17]) some reasonable bounds are available, based on random quantization. (The idea is to upper-bound $\min _{x \in\left(\mathbb{R}^{d}\right)^{N}}\left\|X-\widehat{X}^{x}\right\|_{p}^{p}$ by $\left\|\min _{1 \leq i \leq N}\left|X-Z_{i}\right|\right\|_{p}^{p}$ where the $Z_{i}$ 's are i.i.d. with an appropriate distribution).

Whatsoever, this theorem says that $\min _{x \in\left(\mathbb{R}^{d}\right)^{N}}\left\|X-\widehat{X}^{x}\right\|_{p} \sim C_{X, p, d} N^{\frac{1}{d}}$. This is in accordance with the commonly admitted rates obtained e.g. in Numerical Integration by uniform $N$-tuple methods. In some sense, although optimal quantizers are never uniform square grid (except for the uniform distribution in 1-dimension), optimal quantization provides the best possible "grid method" for a given distribution $\mu$.

\subsubsection{Optimal quantization: how to get it ?}

When $x=\left\{x^{1}, \ldots, x^{N}\right\}$, Equation (16) implies that $\left\|X-\widehat{X}^{x}\right\|_{p}^{p}=\mathbb{E}\left(\min _{1 \leq i \leq N}\left|X-x^{i}\right|^{p}\right)$. The induced symmetric function on $\left(\mathbb{R}^{d}\right)^{N}$ is (Lipschitz) continuous and is denoted $D_{N}^{p}$ from now on $\left({ }^{1}\right)$. One shows (see, e.g., [25] when $p=2$ or [38]) that, if $p>1, D_{N}^{p}$ is continuously differentiable at every $N$-tuple $y \in\left(\mathbb{R}^{d}\right)^{N}$ satisfying $\forall i \neq j, x^{i} \neq x^{j}$ and $\mathbb{P}_{X}\left(\cup_{i=1}^{N} \partial C_{i}(y)\right)=$ 0 . The gradient $\nabla D_{N}^{p}(y)$ is obtained by formal differentiation, that is

$$
\begin{aligned}
\nabla D_{N}^{p} & :=\left(\mathbb{E} \frac{\partial D_{N}^{p}}{\partial x^{i}}(y, X)\right)_{1 \leq i \leq n}=\left(\int_{\mathbb{R}^{d}} \frac{\partial D_{N}^{p}}{\partial x^{i}}(y, u) \mathbb{P}_{X}(d u)\right)_{1 \leq i \leq n} \\
\text { where } \quad \frac{\partial D_{N}^{p}}{\partial x^{i}}(y, u) & :=p \frac{u-x^{i}}{\left|u-x^{i}\right|}\left|u-x^{i}\right|^{p-1} \mathbf{1} C_{i}(y)(u), 1 \leq i \leq n .
\end{aligned}
$$

(The above result still holds when $p=1$ provided that $\mathbb{P}_{Y}$ is continuous.)

So, the gradient of $D_{N}^{p}$ has an integral representation with respect to the distribution of $X$ this strongly suggests to implement a stochastic gradient descent derived from this representation to approximate some (local) minimum of $D_{N}^{p}$ : whenever $d \geq 2$, the implementation of deterministic gradient descent is irrealistic since it would rely on the computation of many

\footnotetext{
${ }^{1}$ The letter $D$ is a reference to the word distortion which used en Information Theory for the $L^{p}$-mean quantization error (to the power $p$ )
} 
integrals with respect $\ldots$ to $\mathbb{P}_{X}$. This stochastic gradient descent is defined as follows: let $\left(\xi^{t}\right)_{t \in \mathbb{N}^{*}}$ a sequence of i.i.d. $\mathbb{P}_{X}$-distributed random variables and let $\left(\delta_{t}\right)_{t \in \mathbb{N}^{*}}$ be a sequence of positive steps satisfying

$$
\sum_{t} \delta_{t}=+\infty \quad \text { and } \quad \sum_{t} \delta_{t}^{2}<+\infty
$$

Then, starting from an initial $N$-tuple $x^{0}$ with $N$ pairwise distinct components, set

$$
x^{t}=x^{t-1}-\delta_{t} \nabla D_{N}^{p}\left(x^{t-1}, \xi^{t}\right)
$$

(this formula a.s. grants by induction that $x^{t}$ has pairwise distinct components). Unfortunately, the usual assumptions that ensure the a.s. convergence of the algorithm (see [19]) are not fulfilled by $D_{N}^{p}$ (see, e.g. [19] or [30] for an overview on Stochastic approximation). To be more specific, let us stress that $D_{N}^{p}(y)$ does not go to infinity as $|y|$ goes to infinity in $\left(\mathbb{R}^{d}\right)^{N}$ and $\nabla D_{N}^{p}$ is clearly not Lipschitz continuous on $\left(\mathbb{R}^{d}\right)^{N}$. Some a.s. convergence results in the Kushner \& Clark sense have been obtained in [38] for compactly supported absolutely continuous distributions $\mathbb{P}_{X}$, mainly in the quadratic case $p=2$ (however, regular a.s. convergence is established when $d=1$ ). In fact the quadratic case is the most commonly implemented for applications and is known as the Competitive Learning vector Quantization (CLVQ) algorithm.

Formula (25) can be developed as follows if one sets $x^{t}:=\left\{x^{1, t}, \ldots, x^{N, t}\right\}$,

Competitive Phase : select $i(t+1) \in \operatorname{argmin}_{i}\left|x^{i, t}-\xi^{t+1}\right|$

LEARNING PHASE $: \quad\left\{\begin{aligned} x^{i(t+1), t+1} & :=x^{i(t+1), t}-\delta_{t+1} \frac{x^{i(t+1), t}-\xi^{t+1}}{\left|x^{i(t+1), t}-\xi^{t+1}\right|}\left|x^{i(t+1), t}-\xi^{t+1}\right|_{(2-1}^{p-1} \\ *[.5 e m] x^{i, t+1} & :=x^{i, t}, i \neq i(t+1) .\end{aligned}\right.$

Furthermore, it is established in [38] that, if $X \in L^{p+\varepsilon}(\varepsilon>0)$, on the event $\left\{x^{t} \rightarrow x^{*}\right\}$

$$
\begin{aligned}
D_{N}^{p, t+1} & :=D_{N}^{p, t}\left(1-\delta_{t+1}\right)+\delta_{t+1} \frac{x^{i(t+1), t}-\xi^{t+1}}{\left|x^{i(t+1), t}-\xi^{t+1}\right|}\left|x^{i(t+1), t}-\xi^{t+1}\right|^{p-1} \stackrel{a . s .}{\rightarrow} \frac{1}{p} D_{N}^{p}\left(x^{*}\right) \\
\pi^{i, t+1} & :=\pi^{i, t}\left(1-\delta_{t+1}\right)+\delta_{t+1} \mathbf{1}_{\{i=i(t+1)\}} \stackrel{a . s}{\longrightarrow} \mathbb{P}_{X}\left(C_{i}\left(x^{*}\right)\right), 1 \leq i \leq N .
\end{aligned}
$$

These "companion" - hence costless - procedures yield the parameters (weights of the Voronoi cells, $L^{p}$-mean quantization error of $x^{*}$ ) necessary to exploit the $N$-tuple $x^{*}$ for numerical purpose. Note that this holds whatever the limiting $N$-tuple $x^{*}$ is: this means that the procedure is consistent.

Concerning practical implementations of the algorithm, it is to be noticed that, when $p=2$ at each, step the $N$-tuple $x^{t+1}$ lives in the convex hull of $x^{t}$ and $\xi^{t+1}$ which has a stabilizing effect on the procedure. One checks on simulation that the CLVQ algorithms does behave better than its non-quadratic counterparts.

Fig.1: Voronoi tessellation of a 300-tuple with the lowest quadratic quantization error for the Normal distribution 


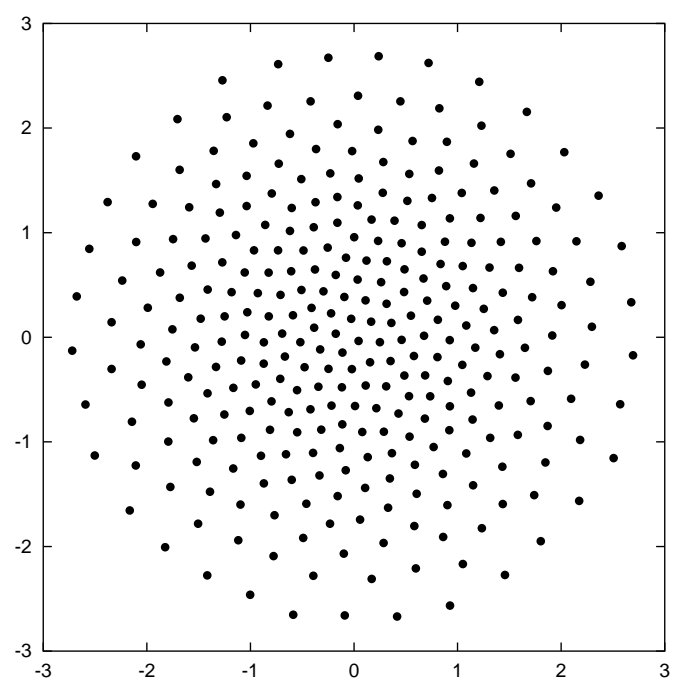

\subsubsection{A priori error bounds}

Next theorem provides a general error bound for $V_{k}-\widehat{v}_{k}\left(\widehat{X}_{k}\right)$ as a function of the quantization errors $\left\|X_{k}-\widehat{X}_{k}\right\|_{p}$ (for any Voronoi quantizers $\widehat{X}_{k}$ ).

Theorem 4 Assume that the coefficients $b$ and $c$ of the diffusion (7) are Lipschitz continuous as well as the obstacle function $h$. Let $\left(X_{k}\right)_{0 \leq k \leq n}$ denote either the sampled diffusion $\left(S_{t_{k}}\right)_{0 \leq k \leq n}$ or the Euler scheme $\left(\bar{S}_{t_{k}}\right)_{0 \leq k \leq n}$. Let $\left(\bar{V}_{k}\right)_{0 \leq k \leq n}$ denote the Snell envelope of the obstacle $\left(h\left(t_{k}, X_{k}\right)\right)_{0 \leq k \leq n}$ and let $\widehat{X}_{k}$ denote a quantizer of $X_{k}$ as defined by (15). Then, for every $p \in[1,+\infty)$, there exists some real constant $K_{b, \sigma, T, p}>0$ such that

$$
\forall n \in \mathbb{N}^{*}, \forall k \in\{0, \ldots, n\}, \quad\left\|V_{k}-\widehat{v}_{k}\left(\widehat{X}_{k}\right)\right\|_{p} \leq K_{b, \sigma, T, p} \sum_{\ell=k}^{n}\left\|X_{\ell}-\widehat{X}_{\ell}\right\|_{p}
$$

where $\left(\widehat{v}_{k}\left(\widehat{X}_{k}\right)\right)_{0 \leq k \leq n}$ denotes the pseudo-Snell envelope of $h\left(t_{k}, X_{k}\right)$ as defined by (17).

The key point in Theorem 4 is that $K_{b, \sigma, T, p}$ does not depend on $n$ One gets rid of $n$ since the Lipschitz coefficient $K^{(n)}$ of the Euler schemes (or the sampled diffusion) satisfies $\lim \sup _{n}\left(K^{(n)}\right)^{n}<+\infty$ (see [4] for details). Combining the bounds obtained in Theorems 1 and 4 will naturally lead to an as small as possible global error. To this end, we need to settle the parameters of the discretization: the number $n$ of discretization times (or "time layers"), the global number $N$ of elementary quantizers, and the size $N_{k}$ of the quantizer $\widehat{X}_{k}$ of the $k^{t h}$ layer (time 0 is excluded since it is perfectly quantized by the single quantizer

INRIA 
$\left.X_{0}:=s_{0}\right)$. Next theorem provides a theoretic answer which minimize the theoretical error bounds. We proceed as follows: $n$ being fixed, the space discretization error is ruled by the right hand side of (30), namely $\sum_{k=1}^{n}\left\|X_{k}-\widehat{X}_{k}\right\|_{p}$. So we need to minimize this quantity for a fixed $n$ and then to make a balance between the resulting space discretization error and the error induced by the time discretization given by Theorem 1 .

To be rigorous, we need to state the theorem one further assumption, called domination assumption, about the distributions of the $X_{k}$ 's. It is based on the $L^{p}$-mean quantization error $\left\|X_{k}-\widehat{X}_{k}\right\|_{p}$. Namely, there exists a random variable $R \in L^{p+\eta}(\eta>0)$ and a sequence $\left(\varphi_{k}\right)_{0 \leq k \leq n}$ such that

$$
\forall k \in\{0, \ldots, n\}, \forall N \in \mathbb{N}^{*}, \quad \underline{D}_{N}^{X_{k}, p} \leq \varphi_{k}^{p} \underline{D}_{N}^{R, p} .
$$

The point is that the distribution of $R$ may depend on $p$ but not on $N$ or $k$.

It is shown in [4] that uniformly elliptic diffusions with smooth and bounded coefficients and their related Euler schemes satisfy the domination property (31) with $\varphi_{k}:=c \sqrt{t_{k}}$. It is shown in the Appendix that, if $q \geq d, \sigma$ is smooth and uniformly elliptic, then the extended Black \& Scholes model (1) is dominated as well.

Theorem 5 Assume that all the assumptions of Theorem 4 hold and that the $\left(X_{k}\right)_{0 \leq k \leq n}$ is dominated in the sense of (31). Let $n, N \in \mathbb{N}^{*}$ and set the size $N_{k}$ of the quantizer of layer $k$ by

$$
N_{k}:=\left\lceil\frac{t_{k}^{\frac{d}{d+1}}}{t_{1}^{\frac{d}{d+1}}+\cdots+t_{k}^{\frac{d}{d+1}}+\cdots+t_{n}^{\frac{d}{d+1}}} N\right\rceil, 1 \leq k \leq n
$$

(then, $N \leq N_{1}+\cdots+N_{n} \leq N+n$ ).

(a) Let $n:=\left\lceil\left(\frac{2 d}{d+1} C_{p}\left(s_{0}\right)\right)^{\frac{2}{3 d+2}} N^{-\frac{1}{2 d+1}}\right\rceil$. Assume furthermore that every quantization $\widehat{X}_{k}$ is $L^{p}$-optimal. Then,

$$
\left\|\mathcal{V}_{t_{k}}-\widehat{V}_{t_{k}}^{n}\right\|_{p} \leq C_{p}^{\prime}\left(1+\left|s_{0}\right|\right) e^{C_{p}^{\prime} T} \frac{n}{(N / n)^{\frac{1}{d}}}=O\left(N^{-\frac{1}{3 d+2}}\right)=O\left(\frac{1}{\sqrt{n}}\right) .
$$

where $\widehat{V}_{k}^{n}$ is the pseudo-Snell envelope of the Euler scheme.

(b) If the obstacle $h$ is semi-convex and if $X_{k}:=S_{t_{k}}$, then set $n:=\left\lceil\left(\frac{2 d}{d+1} C_{p}\left(s_{0}\right)\right)^{\frac{2}{3 d+2}} N^{-\frac{1}{2 d+1}}\right\rceil$. Assume furthermore that every quantization $\widehat{S}_{t_{k}}$ is $L^{p}$-optimal. Then,

$$
\left\|\mathcal{V}_{t_{k}}-\widehat{V}_{t_{k}}^{n}\right\|_{p} \leq C_{p}^{\prime}\left(1+\left|s_{0}\right|\right) e^{C_{p}^{\prime} T} \frac{n}{(N / n)^{\frac{1}{d}}}=O\left(N^{-\frac{1}{2 d+1}}\right)=O\left(\frac{1}{n}\right) .
$$

Remark: The real constant $C_{p}\left(s_{0}\right)$ satisfies $C_{p}\left(s_{0}\right) \leq C_{p}\left(1+\left|s_{0}\right|\right)$ and $C_{p}$ could be calibrated in using a 1-dimension model. 


\section{$4 \quad$ Hedging}

Tackling the question of hedging American options needs to go deeper in financial modelling, at least from a heuristic point of view. So, we will shortly recall the principles that govern the pricing and hedging of American options to justify our approach. First, we come back to the original diffusion model (3) which drives the asset price process $\left(S_{t}\right)$ (with $r=0$ ). Furthermore, we will assume when necessary that $(q \geq d)$ and

$$
\forall x \in \mathbb{R}^{d}, \sigma \sigma^{*}(x) \geq \varepsilon_{0} I_{d}
$$

so that

$$
\varepsilon_{0} \operatorname{Diag}\left(x_{i}^{2}\right) I_{d} \leq c c^{*}(x) \leq\left\|\sigma \sigma^{*}\right\|_{\infty}|x|^{2} I_{d}
$$

\subsection{Hedging continuous time American options}

First we need to come back shortly to classical European option pricing theory. Let $h_{T}$ be a European contingent claim that is a nonnegative $\mathcal{F}_{T}$-measurable $\mathbb{R}^{d}$-valued random vector. Assume for the sake of simplicity that it lies in $L^{2}\left(\mathbb{P}, \mathcal{F}_{T}\right)$. The representation theorem for Brownian martingale shows (see [39]) that

$$
h_{T}=\mathbb{E}\left(h_{T}\right)+\int_{0}^{T} H_{s} \cdot d W_{s}=\mathbb{E}\left(h_{T}\right)+\int_{0}^{T} Z_{s} \cdot d S_{s}
$$

where $H$ is a $d \mathbb{P} d t$-square integrable $\mathcal{F}$-predictable process and $Z_{s}:=\left[c\left(S_{s}\right)^{*}\right]^{-1} H_{s}$. Hence $M_{t}:=\mathbb{E}\left(h_{T} / \mathcal{F}_{t}\right)$ satisfies $M_{t}=M_{0}+\int_{0}^{t} Z_{s} \cdot d S_{s}$.

An analogy with discrete time model shows that the integral $\int_{t}^{T} Z_{s} \cdot d S_{s}$ represents the (algebraic) gain from time $t$ up to time $T$ provided by the strategy $\left(Z_{s}^{i}\right)_{1<i<d}$ (at every time $s \in[t, T]$ the portfolio contains exactly $Z_{s}^{i}$ units of asset $i$ ). So, at time $T$, the value of the portfolio invested in risky assets $S^{1}, \ldots, S^{d}$ is exactly $h_{T}$ monetary units: put some way round, the portfolio $Z_{t}$ replicates the payoff $h_{T}$; so it is natural to define the (theoretical) premium as

$$
\text { Premium }_{t}:=\mathbb{E}\left(h_{T} / \mathcal{F}_{t}\right)=\mathbb{E}\left(h_{T}\right)+\int_{0}^{t} Z_{s} \cdot d S_{s} .
$$

If $h_{T}:=h\left(T, S_{T}\right)$, the Markov property of $\left(S_{t}\right)$ implies that Premium $t:=p\left(t, S_{t}\right)$. If $h$ is regular enough, then $p$ solves the parabolic P.D.E. $\frac{\partial p}{\partial t}+\mathcal{L}_{r, \sigma} p=0, p(T,):.=h(T,$.$) and$ a straightforward application of Ito formula shows that $Z_{t}=\nabla_{x} p\left(t, S_{t}\right)$.

Let us come back to American option pricing. If one defines the premium process $\left(\mathcal{V}_{t}\right)_{t \in[0, T]}$ of an American option by the $\mathbb{P}$-Snell envelope of its payoff process, then this premium process is a supermartingale that can be decomposed as the difference of a martingale $M_{t}$ and a nondecreasing path-continuous process $K_{t}$ i.e., using the representation property of Brownian martingales,

$$
\mathcal{V}_{t}=M_{t}-K_{t}=\mathcal{V}_{0}+\int_{0}^{t} Z_{s} \cdot d S_{s}-K_{t} \quad\left(K_{0}:=0\right)
$$


So, if a trader replicates the European option related to the (unknown) European payoff $M_{T}$ using $Z_{t}$, he is in position to be the counterpart at every time $t$ of the owner of the option in case of exercise since

$$
M_{t}=\mathcal{V}_{t}+K_{t} \geq \mathcal{V}_{t} \geq h_{t}
$$

In case of an optimal exercise of his counterpart he will actually have exactly the payoff at time $t$ since all optimal exercise times occur before the process $K_{t}$ leaves 0 .

If the variational inequality (6) admits a regular enough solution $\nu(t, x)$, then $Z_{t}=$ $\nabla_{x} \nu\left(t, S_{t}\right)$. In most deterministic numerical methods, the approximation of such a derivative is usually less accurate than that of the function $\nu$ itself. So, it is hopeless to implement such methods to this end as soon as the dimension $d$ is greater than 3 .

\subsection{Hedging Bermuda options}

Let $\left(V_{t_{k}}^{n}\right)_{0 \leq k \leq n}$ denote the theoretical premium process of the Bermuda option related to $\left(h\left(t_{k}, S_{t_{k}}\right)\right)_{0 \leq k \leq n}$. It is a $\left(\mathcal{F}_{t_{k}}\right)_{0 \leq k \leq n}$-supermartingale defined as a Snell envelope by

$$
V_{t_{k}}^{n}:=\operatorname{ess} \sup \left\{\mathbb{E}_{t_{k}}\left(h\left(\tau, S_{\tau}\right)\right), \tau \in \Theta_{k}^{n}\right\}
$$

where $\Theta_{k}^{n}$ denotes the set of $\left\{t_{k}, \ldots, t_{n}\right\}$-valued $\mathcal{F}$-stopping times.

Then, the $\mathcal{F}_{t_{k}}$-Doob decomposition of $\left(V_{t_{k}}^{n}\right)$ as a the $\left(\mathcal{F}_{t_{k}}\right)$-supermartingale yield:

$$
V_{t_{k}}^{n}=M_{k}^{n}-A_{k}^{n},
$$

where $\left(M_{t_{k}}^{n}\right)$ is a $\mathcal{F}_{t_{k}}-L^{2}$-martingale and $\left(A_{t_{k}}^{n}\right)$ is a non-decreasing integrable $\mathcal{F}_{t_{k}}$-predictable process $\left(A_{0}^{n}:=0\right)$. In fact, the increment of $A_{k}^{n}$ can easily be specified since

$$
\Delta A_{k}^{n}:=A_{k}^{n}-A_{k-1}^{n}=V_{t_{k-1}}^{n}-\mathbb{E}_{t_{k-1}} V_{t_{k}}^{n}=\left(h\left(t_{k-1}, S_{t_{k-1}}\right)-\mathbb{E}_{t_{k-1}} V_{t_{k}}^{n}\right)_{+} .
$$

The representation theorem applied on each time interval $\left[t_{k}, t_{k+1}\right], k=0, \ldots, n$ then yields a $\mathcal{F}$-progressively measurable process $\left(Z_{s}^{n}\right)_{s \in[0, T]}$ satisfying

$$
M_{k}^{n}:=\int_{0}^{t_{k}} Z_{s}^{n} \cdot d S_{s}, \quad 0 \leq k \leq n, \quad \text { with } \quad \mathbb{E} \int_{0}^{T}\left|c^{*}\left(S_{s}\right) Z_{s}^{n}\right|^{2} d s<+\infty
$$

(keep in mind that $<\int_{0}^{t_{k}} U_{s} \cdot d S_{s}>_{t}=\int_{0}^{t_{k}}\left|c^{*}\left(S_{s}\right) U_{s}\right|^{2} d s$ ).

Now, in such a setting, continuous time hedging of a Bermuda option is irrealistic since the approximation of an American by a Bermuda option is directly motivated by discrete time hedging (at times $t_{k}$ ). So, it seems natural to look for what a trader can do best when hedging only at times $t_{k}$. This leads to consider the closed subspace $\mathcal{P}_{n}$ of $L^{2}\left(c^{*}(S). d \mathbb{P} . d t\right)$ defined by

$\mathcal{P}_{n}=\left\{\left(\zeta_{s}\right)_{s \in[0, T]}, \zeta_{s}:=\zeta_{t_{k}}, s \in\left[t_{k}, t_{k+1}\right), \zeta_{t_{k}} \mathcal{F}_{t_{k}}\right.$-measurable, $\left.\mathbb{E} \int_{0}^{T}\left|c^{*}\left(S_{s}\right) \zeta_{s}\right|^{2} d s<+\infty\right\}$. 
and the induced orthogonal projection $\operatorname{proj}_{n}$ onto $\mathcal{P}_{n}$ (for notational simplicity a process $\zeta \in \mathcal{P}_{n}$ will be often referred as $\left.\left(\zeta_{t_{k}}\right)_{0 \leq k \leq n}\right)$. In particular, for every $U \in L^{2}\left(c^{*}(S). d \mathbb{P} . d t\right)$

$$
\left\|c^{*}(S .) \operatorname{proj}_{n}(U) .\right\|_{L^{2}(d \mathbb{P} . d t)} \leq\left\|c^{*}(S .) U .\right\|_{L^{2}(d \mathbb{P} . d t)} .
$$

Doing so, we follow classical ideas introduced by by Follmer \& Sonderman ([21]) for hedging purpose in incomplete markets (see also [10]). One checks that $\mathcal{P}_{n}$ is isometric with the set of square integrable stochastic integrals with respect to $\left(S_{t_{k}}\right)_{0 \leq k \leq n}$, namely

$$
\left\{\sum_{k=1}^{n} \zeta_{t_{k}} . \Delta S_{t_{k+1}},\left(\zeta_{t_{k}}\right)_{0 \leq k \leq n} \in \mathcal{P}_{n}\right\} .
$$

Computing $\operatorname{proj}_{n}\left(Z_{.}^{n}\right)$ amounts to minimizing $\mathbb{E}\left(\sum_{k=1}^{n} \int_{t_{k}}^{t_{k+1}}\left|c^{*}\left(S_{s}\right)\left(Z_{s}^{n}-\zeta_{t_{k}}\right)\right|^{2} d s\right)$ over $\left(\zeta_{k}\right)_{0 \leq k \leq n} \in \mathcal{P}_{n}$. Setting $\zeta_{t_{k}}^{n}:=\operatorname{proj}_{n}\left(Z^{n}\right)$ and standard computations yield

$$
\begin{aligned}
\zeta_{t_{k}}^{n} & :=\left(\mathbb{E}_{t_{k}} \int_{t_{k}}^{t_{k+1}} c c^{*}\left(S_{s}\right) d s\right)^{-1} \mathbb{E}_{t_{k}}\left(\int_{t_{k}}^{t_{k+1}} c c^{*}\left(S_{s}\right) Z_{s}^{n} d s\right) \\
& =\left(\mathbb{E}_{t_{k}} \Delta S_{t_{k+1}}\left(\Delta S_{t_{k+1}}\right)^{*}\right)^{-1} \mathbb{E}_{t_{k}}\left(\Delta M_{k+1}^{n} \Delta S_{t_{k+1}}\right) \\
& =\left(\mathbb{E}_{t_{k}} \Delta S_{t_{k+1}}\left(\Delta S_{t_{k+1}}\right)^{*}\right)^{-1} \mathbb{E}_{t_{k}}\left(\Delta V_{t_{k+1}}^{n} \Delta S_{t_{k+1}}\right) .
\end{aligned}
$$

The last equality follows from the fact that $A_{k-1}^{n}$ is $\mathcal{F}_{t_{k-1}}$-measurable and from the martingale property of $\left(S_{t_{k}}\right)$. The increment

$$
\Delta R_{t_{k+1}}^{n}:=\int_{t_{k}}^{t_{k+1}}\left(Z_{s}^{n}-\zeta_{t_{k}}^{n}\right) \cdot d S_{s}=\Delta M_{k+1}^{n}-\zeta_{t_{k}}^{n} \cdot \Delta S_{t_{k+1}}
$$

represents the hedging default induced by using $\zeta_{t_{k}}^{n}$ instead of $Z^{n}$. The sequence $\left(\Delta R_{t_{k}}^{n}\right)_{0 \leq k \leq n}$ is a $\mathcal{F}_{t_{k}}$-martingale increment process, singular with respect to $\left(S_{t_{k}}\right)_{0 \leq k \leq n}$ since $\mathbb{E}_{t_{k}}\left(\Delta R_{t_{k+1}} \Delta S_{t_{k+1}}\right)=0$. It is possible to define the local residual risk by

$$
\mathbb{E}_{t_{k}}\left|\Delta R_{t_{k+1}}^{n}\right|^{2}=\mathbb{E}_{t_{k}}\left(\int_{t_{k}}^{t_{k+1}}\left|c^{*}\left(S_{s}\right)\left(Z_{s}^{n}-\zeta_{t_{k}}\right)\right|^{2} d s\right), k \in\{0, \ldots, n-1\}
$$

A little algebra yields the following, more appropriate for quantization purpose:

$$
\mathbb{E}_{t_{k}}\left|\Delta R_{t_{k+1}}^{n}\right|^{2}=\mathbb{E}_{t_{k}}\left|\Delta V_{t_{k+1}}^{n} \mathbb{E}_{t_{k}} \Delta V_{t_{k+1}}^{n}\right|^{2}-\left(\mathbb{E}_{t_{k}} \Delta S_{t_{k+1}} \Delta S_{t_{k+1}}^{*}\right)^{-1}\left(\mathbb{E}_{t_{k}} \Delta V_{t_{k+1}}^{n} \Delta S_{t_{k+1}}\right)^{2}
$$

Formulae (40) or (42), based on $S_{t_{k}}$ and $V_{t_{k}}^{n}$ have natural approximations by quantization. On the other hand, (39) and (42) are more appropriate to produce some a priori error bounds (when simulation of the diffusion is possible). 


\subsection{Hedging Bermuda option on the Euler scheme}

When the diffusion cannot be easily simulated, we substitute the (continuous time) Euler scheme defined by

$$
\forall t \in\left[t_{k}, t_{k+1}\right), \quad \bar{S}_{t}=\bar{S}_{t_{k}}+c\left(\bar{S}_{t_{k}}\right)\left(W_{t}-W_{t_{k}}\right), \quad \bar{S}_{0}:=s_{0}>0 .
$$

This process is $\mathbb{P}$-a.s. defined since it is a.s. nonzero (but it may become negative contrarily to the original diffusion). Then, mimicking the above subsection, leads to define some processes $\bar{Z}^{n}, \bar{M}^{n}$ and $\bar{A}^{n}$ by

$$
\begin{aligned}
\bar{V}_{t_{k}}^{n} & :=\bar{M}_{k}^{n}-\bar{A}_{k}^{n} \quad \text { (Doob decomposition) } \\
\bar{M}_{k}^{n} & :=\int_{0}^{t_{k}} \bar{Z}_{s}^{n} c\left(\bar{S}_{\underline{s}}\right) d \bar{W}_{s}=\int_{0}^{t_{k}} \bar{Z}_{s}^{n} d \bar{S}_{s} \quad\left(\text { with } \underline{s}=t_{i}, s \in\left[t_{i}, t_{i+1}\right)\right) \\
\Delta \bar{A}_{k}^{n} & :=\bar{A}_{k}^{n}-\bar{A}_{k-1}^{n}=\bar{V}_{t_{k-1}}^{n}-\mathbb{E}_{t_{k-1}} \bar{V}_{t_{k}}^{n}=\left(h\left(t_{k-1}, \bar{S}_{t_{k-1}}\right)-\mathbb{E}_{t_{k-1}} \bar{V}_{t_{k}}^{n}\right)_{+} .
\end{aligned}
$$

and $\bar{A}_{0}^{n}:=0$. The (simpler) formulae for the hedging process hold

$$
\bar{\zeta}_{t_{k}}^{n}:=\left(\mathbb{E}_{t_{k}} \Delta \bar{S}_{t_{k+1}} \Delta \bar{S}_{t_{k+1}}^{*}\right)^{-1} \mathbb{E}_{t_{k}}\left(\Delta \bar{V}_{t_{k+1}}^{n} \Delta \bar{S}_{t_{k+1}}\right)=\frac{1}{\Delta t_{k+1}} \mathbb{E}_{t_{k}} \int_{t_{k}}^{t_{k+1}} \bar{Z}_{s}^{n} d s
$$

The related hedging default and local residual risk are defined by mimicking (42) and (43):

$$
\begin{aligned}
& \Delta \bar{R}_{t_{k+1}}^{n}:=\int_{t_{k}}^{t_{k+1}}\left(\bar{Z}_{s}^{n}-\bar{\zeta}_{t_{k}}^{n}\right) \cdot d \bar{S}_{s}=\Delta M_{k+1}^{n}-\bar{\zeta}_{t_{k}}^{n} \cdot \Delta \bar{S}_{t_{k+1}} \\
& \mathbb{E}_{t_{k}}\left|\Delta \bar{R}_{t_{k+1}}^{n}\right|^{2}:=\mathbb{E}_{t_{k}}\left|\Delta \bar{V}_{t_{k+1}}^{n} \mathbb{E}_{t_{k}} \Delta \bar{V}_{t_{k+1}}^{n}\right|^{2}-\left(\mathbb{E}_{t_{k}} \Delta \bar{S}_{t_{k+1}} \Delta \bar{S}_{t_{k+1}}^{*}\right)^{-1}\left(\mathbb{E}_{t_{k}} \Delta \bar{V}_{t_{k+1}}^{n} \Delta \bar{S}_{t_{k+1}}()^{2}\right)
\end{aligned}
$$

\subsection{Quantized hedging and local residual risks}

The quantized formulae for strategies and residual risks are simply derived from formulae (40) or (44) by replacing $S_{t_{k}}\left(\bar{S}_{t_{k}}\right.$ respectively) by their quantization $\widehat{S}_{t_{k}}$ ( $\widehat{\bar{S}}_{t_{k}}$ respectively) and $V_{k}^{n}:=v_{k}^{n}\left(S_{t_{k}}\right)$ by $\widehat{V}_{k}^{n}:=\widehat{v}_{k}^{n}\left(\widehat{S}_{t_{k}}\right)\left(\widehat{V}_{k}^{n}:=\widehat{v}_{k}^{n}\left(\widehat{\bar{S}}_{t_{k}}\right)\right.$ respectively). It follows from section 3 that $V_{t_{k}}^{n}:=v_{k}\left(S_{t_{k}}\right)$ is approximated by $\widehat{v}_{k}^{n}\left(\widehat{S}_{t_{k}}\right)$. So, one sets (for the diffusion)

$$
\begin{aligned}
\widehat{\zeta}_{k}^{n} & :=\frac{n}{T}\left(c c^{*}\left(\widehat{S}_{t_{k}}\right)\right)^{-1} \widehat{\mathbb{E}}_{k}\left(\left(\widehat{v}_{k+1}^{n}\left(\widehat{S}_{t_{k+1}}\right)-\widehat{v}_{k}^{n}\left(\widehat{S}_{t_{k}}\right)\right)\left(\widehat{S}_{t_{k+1}}-\widehat{S}_{t_{k}}\right)\right) . \\
\left|\Delta \widehat{R}_{t_{k+1}}^{n}\right|^{2} & :=\mathbb{E}_{t_{k}}\left|\Delta \widehat{V}_{t_{k+1}}^{n}-\mathbb{E}_{t_{k}} \Delta \widehat{V}_{t_{k+1}}^{n}\right|^{2}-\left(\mathbb{E}_{t_{k}} \Delta \widehat{S}_{t_{k+1}} \Delta \widehat{S}_{t_{k+1}}^{*}\right)^{-1}\left(\mathbb{E}_{t_{k}} \Delta \widehat{V}_{t_{k+1}}^{n} \Delta \widehat{S}_{t_{k+1}}\right)^{2}
\end{aligned}
$$


One derives their counterparts $\widehat{\bar{\zeta}}_{k}^{n},\left|\Delta \widehat{\bar{R}}_{t_{k+1}}^{n}\right|^{2}$ for the Euler scheme by analogy. The point to be noticed is that computing $\widehat{\zeta}_{t_{k}}^{n}$ or $\widehat{\bar{\zeta}}_{k}^{n}$ at a given point $x_{i}^{k}$ of the $k^{\text {th }}$ layer requires to invert only one matrix which does not cost much.

\section{Convergence of the hedging strategies and rates}

This section is devoted to the evaluation of the different errors (quantization, residual risks) induced by space and time discretization.

\subsection{From Bermuda to America}

First, one extends the definition of $V_{t}^{n}$ at any time $t \in[0, T]$ by setting

$$
V_{t}^{n}:=V_{t_{k}}^{n}+\int_{t_{k}}^{t} Z_{s}^{n} d S_{s}=V_{t_{k+1}}^{n}-\int_{t}^{t_{k+1}} Z_{s}^{n} d S_{s}+\Delta A_{k+1}^{n}, t \in\left[t_{k}, t_{k+1}\right) .
$$

This definition implies that, for every $k \in\{0, \ldots, n\}$, the left-limit of $V^{n}$ satisfies

$$
V_{t_{k}-}^{n}=V_{t_{k}}^{n}+\Delta A_{k+1}^{n} \text {. }
$$

Proposition 2 Assume that the payoff process $h_{t}=h\left(t, S_{t}\right)$ where $h$ is a semi-convex function. Assume that the diffusion coefficient $c$ is Lipschitz continuous.

(a) For every $k \in\{0, \ldots, n\}, V_{t_{k}}^{n} \leq \mathcal{V}_{t_{k}}$ and for every $t \in\left(t_{k}, t_{k+1}\right), \quad\left(V_{t}^{n}-\mathcal{V}_{t}\right)_{+} \leq \Delta A_{k+1}^{n}$.

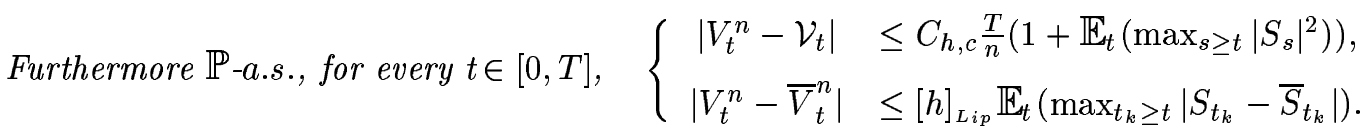

(b) The following bound holds for the hedging strategies (in the "cc* metric")

$$
\left.\mathbb{E}\left(\int_{0}^{T}\left|c^{*}\left(S_{s}\right)\left(Z_{s}-Z_{s}^{n}\right)\right|^{2} d s\right)+\left.\mathbb{E}\left(\int_{0}^{T} \mid c^{*}\left(S_{s}\right) Z_{s}^{n}-c^{*}\left(\bar{S}_{s}\right) \bar{Z}_{s}^{n}\right)\right|^{2} d s\right) \leq C_{h, c} \frac{T}{n} .
$$

Proof: $(a)$ The inequality between $V^{n}$ and $\mathcal{V}$ at times $t_{k}$ is obvious since $\mathcal{V}_{t}$ is defined as a supremum over a larger set of stopping times than $V_{t_{k}}^{n}$. Then, using the supermartingale property of $\mathcal{V}$, equality (49) and Jensen inequality yields

$\left(V_{t}^{n}-\mathcal{V}_{t}\right)_{+} \leq\left(\mathbb{E}_{t}\left(V_{t_{k+1}}^{n}\right)+\Delta A_{k+1}^{n}-\mathbb{E}_{t}\left(\mathcal{V}_{t_{k+1}}\right)\right)_{+} \leq \mathbb{E}_{t}\left(\left(V_{t_{k+1}}^{n}-\mathcal{V}_{t_{k+1}}+\Delta A_{k+1}^{n}\right)_{+}\right) \leq \Delta A_{k+1}^{n}$

Now, using the expression (36) for $\Delta A_{k+1}^{n}$ and $V_{t_{k}}^{n} \geq h\left(t_{k+1}, S_{t_{k+1}}\right)$ imply

$$
\Delta A_{k+1}^{n}=\left(h\left(t_{k}, S_{t_{k}}\right)-\mathbb{E}_{t_{k}} V_{t_{k+1}}^{n}\right)_{+} \leq\left(h\left(t_{k}, S_{t_{k}}\right)-\mathbb{E}_{t_{k}} h\left(t_{k+1}, S_{t_{k+1}}\right)\right)_{+}
$$


We need at this stage to use the regularity of $h$ (semi-convex Lipschitz continuous)

$$
\begin{aligned}
h\left(t_{k}, S_{t_{k}}\right)-h\left(t_{k+1}, S_{t_{k+1}}\right) & =h\left(t_{k}, S_{t_{k+1}}\right)-h\left(t_{k+1}, S_{t_{k+1}}\right)+h\left(t_{k}, S_{t_{k}}\right)-h\left(t_{k}, S_{t_{k+1}}\right) \\
& \leq[h]_{L i p} \Delta t_{k+1}-\delta_{h}\left(t_{k}, S_{t_{k}}\right) \cdot\left(S_{t_{k+1}}-S_{t_{k}}\right)+\rho_{h}\left(S_{t_{k+1}}-S_{t_{k}}\right)^{2} .
\end{aligned}
$$

Hence $h\left(t_{k}, S_{t_{k}}\right)-\mathbb{E}_{t_{k}} h\left(t_{k+1}, S_{t_{k+1}}\right) \leq[h]_{L i p} \Delta t_{k+1}+\rho_{h} \mathbb{E}_{t_{k}}\left|S_{t_{k+1}}-S_{t_{k}}\right|^{2}$

$$
\begin{aligned}
& \leq[h]_{L i p} \Delta t_{k+1}+\rho_{h} \mathbb{E}_{t_{k}} \int_{t_{k}}^{t_{k+1}} \operatorname{Tr}\left(c c^{*}\right)\left(S_{s}\right) d s \\
& \leq[h]_{L i p} \Delta t_{k+1}+C \rho_{h} \Delta t_{k+1}\left(1+\mathbb{E}_{t}\left(\max _{s \geq t_{k}}\left|S_{s}\right|^{2}\right)\right) \\
& \leq C_{c, h} \frac{T}{n}\left(1+\mathbb{E}_{t_{k}}\left(\max _{s \geq t_{k}}\left|S_{s}\right|^{2}\right)\right) \text { for some constant } C_{h, c}>0 .
\end{aligned}
$$

Finally, it yields

$$
\Delta A_{k+1}^{n} \leq C_{c, h} \frac{T}{n}\left(1+\mathbb{E}_{t_{k}}\left(\max _{s \geq t_{k}}\left|S_{s}\right|^{2}\right)\right) .
$$

To complete the inequality for $\left|\mathcal{V}_{t}-V_{t}^{n}\right|$, one first notice that, if $t \in\left[t_{k}, t_{k+1}\right)$

$$
V_{t}^{n}=V_{t_{k+1}}^{n}-\int_{t}^{t_{k+1}} Z_{s}^{n} d S_{s}+\Delta A_{k+1}^{n} \leq h\left(t_{k+1}, S_{t_{k+1}}\right)-\int_{t}^{t_{k+1}} Z_{s}^{n} d S_{s}
$$

so that $V_{t}^{n}=\mathbb{E}_{t}\left(V_{t}^{n}\right) \geq \mathbb{E}_{t}\left(h\left(t_{k+1}, S_{t_{k+1}}\right)\right)=h\left(t, S_{t}\right)+\mathbb{E}_{t}\left(h\left(t_{k+1}, S_{t_{k+1}}\right)-h\left(t, S_{t}\right)\right)$.

Using again he semi-convexity property of $h$ at $\left(t, S_{t}\right)$ finally yields that

$$
V_{t}^{n}+C_{c, h} \frac{T}{n}\left(1+\mathbb{E}_{t}\left(\max _{s \geq t}\left|S_{s}\right|^{2}\right)\right) \geq h\left(t, S_{t}\right) .
$$

As it is a supermartingale as well, it necessarily satisfies

$$
\mathbb{P} \text {-a.s. } \quad V_{t}^{n}+C_{c, h} \frac{T}{n}\left(1+\mathbb{E}_{t}\left(\max _{s \geq t}\left|S_{s}\right|^{2}\right)\right) \geq \operatorname{Snell}\left(h\left(t, S_{t}\right)\right)=\mathcal{V}_{t}
$$

which yields the expected result. The second inequality is obvious once noticed

$$
\left|V_{t}^{n}-\bar{V}_{t}^{n}\right| \leq \max _{t_{k} \geq t}\left|h\left(t_{k}, S_{t_{k}}\right)-h\left(t_{k}, \bar{S}_{t_{k}}\right)\right| \leq[h]_{L_{i p}} \max _{t_{k} \geq t}\left|S_{t_{k}}-\bar{S}_{t_{k}}\right| .
$$

(b) One considers the càdlàg semi-martingale $\mathcal{V}_{t}-V_{t}^{n}=\mathcal{V}_{0}-V_{0}^{n}+\int_{0}^{t}\left(Z_{s}-Z_{s}^{n}\right) \cdot d S_{s}-\left(K_{t}-A_{\underline{t}}^{n}\right)$ where $\underline{t}:=k$ on $\left[t_{k}, t_{k+1}\right)$. It follows from Itô formula for jump processes that

$$
\begin{aligned}
\int_{0}^{T}\left|c^{*}\left(S_{s}\right)\left(Z_{s}-Z_{s}^{n}\right)\right|^{2} d s & +\sum_{t_{k} \leq T}\left(\Delta A_{t_{k}}^{n}\right)^{2}+\left(\mathcal{V}_{t}-V_{t}^{n}\right)^{2} \\
& =-2 \int_{0}^{T}\left(\mathcal{V}_{s}-V_{s-}^{n}\right)\left(Z_{s}-Z_{s}^{n}\right) \cdot d S_{s}+2 \int_{t}^{T}\left(\mathcal{V}_{s}-V_{s-}^{n}\right) d\left(K_{s}-A_{\underline{t}}^{n}\right) .
\end{aligned}
$$




$$
\text { Now } \begin{aligned}
\int_{0}^{T}\left(\mathcal{V}_{s}-V_{s-}^{n}\right) d\left(K_{s}-A_{\underline{s}}^{n}\right) & =\int_{0}^{T}\left(\mathcal{V}_{s}-V_{s-}^{n}\right) d K_{s}+\int_{t}^{T}\left(V_{s-}^{n}-\mathcal{V}_{s}\right) d A_{\underline{s}}^{n} \\
& \leq \int_{0}^{T}\left(\mathcal{V}_{s}-V_{s}^{n}\right) d K_{s}+\sum_{t_{k} \leq T}\left(\Delta A_{k}^{n}\right)^{2}
\end{aligned}
$$

since $V_{t_{k}-}^{n}=V_{t_{k}}^{n}+\Delta A_{k}^{n} \leq \mathcal{V}_{t_{k}}+\Delta A_{k}^{n}$. This yields, using the inequality obtained in (a) and $(52)$,

$$
\begin{aligned}
\int_{0}^{T}\left(\mathcal{V}_{s}-V_{s-}^{n}\right) d\left(K_{s}-A_{\underline{s}}^{n}\right) & \leq C_{h, c} \frac{T}{n} \int_{0}^{T}\left(1+\mathbb{E}_{s} \max _{u \geq s}\left|S_{u}\right|^{2}\right) d K_{s}+A_{n}^{n} \max _{t<t_{k} \leq T} \Delta A_{k}^{n} \\
& \leq C_{h, c} \frac{T}{n}\left(K_{T}\left(1+\sup _{s \in[0, T]}\left(\mathbb{E}_{s} \max _{u \geq s}\left|S_{u}\right|^{2}\right)\right)+\left(1+\sup _{s \in[0, T]}\left(\mathbb{E}_{s} \max _{u \geq s}\left|S_{u}\right|^{2}\right)\right)^{2}\right) .
\end{aligned}
$$

One checks that $\int_{0}^{t}\left(\mathcal{V}_{s}-V_{s}^{n}\right)\left(Z_{s}-Z_{s}^{n}\right) d S_{s}$ is a true martingale so that

$$
\mathbb{E}\left(\int_{0}^{T}\left|c^{*}\left(S_{s}\right)\left(Z_{s}-Z_{s}^{n}\right)\right|^{2} d s\right) \leq C_{h, c} \frac{T}{n}\left(\left\|K_{T}\right\|_{2}+1\right)\left(1+\left\|\max _{s \in[0, T]}\left|S_{s}\right|^{2}\right\|_{2}\right) .
$$

Now $K_{T} \in L^{2}$ since $0 \leq K_{T} \leq \mathcal{V}_{0}+\int_{0}^{T} Z_{s} d S_{s}$ which yields the expected result.

The inequality involving the Euler scheme is obtained following the same approach using now $V^{n}-\bar{V}^{n}$.

$$
\begin{aligned}
\mathbb{E} \int_{0}^{T}\left|c^{*}\left(S_{s}\right) Z_{s}^{n}-c^{*}\left(\bar{S}_{s}\right) \bar{Z}_{s}^{n}\right|^{2} d s & \leq 2 \mathbb{E} \int_{0}^{T}\left(V_{s}^{n}-\bar{V}_{s}^{n}\right) d\left(K_{s}^{n}-\bar{K}_{s}^{n}\right)+\mathbb{E}\left(h\left(T, S_{T}\right)-h\left(T, \bar{S}_{T}\right)\right)^{2} \\
& \leq 2[h]_{L i p} \mathbb{E} \int_{0}^{T} \mathbb{E}_{s}\left(\max _{t_{k} \geq s}\left|S_{t_{k}}-\bar{S}_{t_{k}}\right|\right) d\left(K_{s}^{n}+\bar{K}_{s}^{n}\right)+[h]_{L i p}^{2}\left\|S_{T}-\bar{S}_{T}\right\|_{2}^{2} \\
& \leq C \mathbb{E}\left(\sup _{t \in[0, T]} \mathbb{E}_{t}\left(\max _{t_{k} \geq t}\left|S_{t_{k}}-\bar{S}_{t_{k}}\right|\right)\left(K_{T}^{n}+\bar{K}_{T}^{n}\right)\right)+C\left\|S_{T}-\bar{S}_{T}\right\|_{2}^{2} \\
& \leq C\left\|\sup _{t \in[0, T]} \mathbb{E}_{t} \max _{t_{k} \geq t}\left|S_{t_{k}}-\bar{S}_{t_{k}}\right|\right\|\left(\left\|K_{T}^{n}\right\|_{2}+\left\|\bar{K}_{T}^{n}\right\|_{2}\right)+C\left\|S_{T}-\bar{S}_{T}\right\|_{2}^{2} \\
& \leq C_{h, c} \frac{T}{n}\left(\left\|K_{T}^{n}\right\|_{2}+\left\|\bar{K}_{T}^{n}\right\|_{2}+1\right) .
\end{aligned}
$$

Now $\left\|K_{T}^{n}\right\|_{2} \leq\left\|V_{0}^{n}\right\|_{2}+\left\|\int_{0}^{T}\left(Z_{s}-Z_{s}^{n}\right) d S_{s}\right\|_{2} \leq C_{1}\left(1+\left\|\sup _{s \in[0, T]}\left|S_{s}\right|\right\|_{2}\right)+O(1 / n)$, hence $\sup _{n}\left\|K_{T}^{n}\right\|_{2}<+\infty$. Concerning $\bar{K}_{T}^{n}$ one has

$\left.\left\|K_{T}^{n}-\bar{K}_{T}^{n}\right\|_{2} \leq\left\|V_{0}^{n}\right\|_{2}+\left\|\bar{V}_{0}^{n}\right\|_{2}+\| \int_{0}^{T} Z_{s}^{n} d S_{s}-\int_{0}^{T} \bar{Z}_{s}^{n} d \bar{S}_{s}\right) \|_{2} \leq C+O(1 / \sqrt{n}) \quad$ by $(54)$ 
so that $\sup _{n}\left\|\bar{K}_{T}^{n}\right\|_{2}<+\infty$. Plugging this back in (54) completes the proof. $\diamond$

We are now in position to get a first result about the control of residual risks induced by the use of discrete time hedging strategies. It shows that this control is essentially ruled by the path-regularity of the process $Z$.

Theorem 6 If $h$ is semi-convex and $h$ and $c$ are Lipschitz continuous, then

$$
\left\|c^{*}(S .)\left(Z .-\zeta_{.}^{n}\right)\right\|_{L^{2}(d \mathbb{P} \otimes d t)} \leq\left\|c^{*}(S .)\left(Z .-\zeta_{.}\right)\right\|_{L^{2}(d \mathbb{P} \otimes d t)}+\frac{C}{\sqrt{n}} \quad \text { where } \zeta:=\operatorname{proj}_{n}(Z)
$$

is the projection of $Z$ on $\mathcal{P}_{n}$. Furthermore $\left\|c^{*}(S .)\left(Z .-\zeta_{\text {. }}\right)\right\|_{L^{2}(d \mathbb{P} \otimes d t)}$ goes to 0 as $n$ goes to 0 . So, this term which depends on the path-regularity of $Z_{s}$, rules the rate of convergence.

Proof: Minkowski inequality yields

$$
\left\|c^{*}(S .)\left(Z_{s}-\zeta_{.}^{n}\right)\right\|_{L^{2}(d \mathbb{P} \otimes d t)} \leq\left\|c^{*}(S .)\left(Z .-\zeta_{.}\right)\right\|_{L^{2}(d \mathbb{P} \otimes d t)}+\left\|c^{*}(S .)\left(\zeta .-\zeta_{.}^{n}\right)\right\|_{L^{2}(d \mathbb{P} \otimes d t)} .
$$

Now $\zeta .-\zeta^{n}=\operatorname{proj}_{n}\left(Z .-Z^{n}\right)$ so that by Inequality (51) in Proposition $2(b)$,

$$
\left\|c^{*}(S .)\left(\zeta .-\zeta_{.}^{n}\right)\right\|_{L^{2}(d \mathbb{P} \otimes d t)} \leq\left\|c^{*}(S .)\left(Z .-Z_{.}^{n}\right)\right\|_{L^{2}(d \mathbb{P} \otimes d t)} \leq \frac{C}{\sqrt{n}} .
$$

Now, let $F$ be a bounded adapted continuous-path process. Set $\Phi_{s}:=\frac{n}{T} \int_{t_{k}}^{t_{k+1}} F_{u} d u, s \in$ $\left[t_{k}, t_{k+1}\right)$. Using the properties of $\operatorname{proj}_{n}$, one gets

$$
\begin{aligned}
\left\|c^{*}(S .)(Z .-\zeta .)\right\|_{L^{2}(d \mathbb{P} \otimes d t)} & \leq 2\left\|c^{*}(S .)(Z .-F .)\right\|_{L^{2}(d \mathbb{P} \otimes d t)}+\left\|c^{*}(S .)\left(F .-\operatorname{proj}_{n}(F) .\right)\right\|_{L^{2}(d \mathbb{P} \otimes d t)} \\
& \leq 2\left\|c^{*}(S .)(Z .-F .)\right\|_{L^{2}(d \mathbb{P} \otimes d t)}+\left\|c^{*}(S .)(F .-\Phi .)\right\|_{L^{2}(d \mathbb{P} \otimes d t)} \\
& \leq 2\left\|c^{*}(S .)(Z .-F .)\right\|_{L^{2}(d \mathbb{P} \otimes d t)}+\left\|\int_{0}^{T}\right\| c\left(S_{s}\right)\left\|^{2} d s\left(w\left(F, \frac{T}{n}\right) \wedge 2\|F\|_{\infty}\right)^{2}\right\|_{L^{2}(\mathbb{P})}
\end{aligned}
$$

where $w(F, \delta)$ denotes the uniform continuity modulus of $F$. One concludes using that the space $L^{\infty}\left(c^{*}\left(S_{t}\right) d \mathbb{P} d t\right)$ is everywhere dense in $L^{2}\left(c^{*}\left(S_{t}\right) d \mathbb{P} d t\right)$.

\subsection{Hedging error induced by the (quadratic) quantization}

We will focus on the error at time $t_{0}=0$.

Proposition 3 If $\sigma$ Lipschitz continuous, bounded and uniformly elliptic and if $h$ is semiconvex and Lipschitz continuous, then

$$
\left|\zeta_{0}^{n}-\widehat{\zeta}_{0}^{n}\right| \leq C\left(1+\left|s_{0}\right|\right) \mid \frac{n^{\frac{3}{2}}}{(N / n)^{\frac{1}{d}}} .
$$


Proof: The hedging vectors $\zeta_{0}^{n}$ and $\hat{\zeta}_{0}^{n}$ satisfy respectively

$$
\begin{aligned}
& \left(\mathbb{E}\left(\Delta S_{t_{1}} \Delta S_{t_{1}}^{*}\right)\right) \zeta_{0}^{n}=\mathbb{E}\left(\left(V_{1}^{n}-V_{0}^{n}\right) \Delta S_{t_{1}}\right) \\
& \left(\mathbb{E}\left(\Delta \widehat{S}_{t_{1}} \widehat{\Delta} S_{t_{1}}^{*}\right)\right) \widehat{\zeta}_{0}^{n}=\mathbb{E}\left(\left(\widehat{V}_{1}^{n}-\widehat{V}_{0}^{n}\right) \Delta \widehat{S}_{t_{1}}\right)
\end{aligned}
$$

where $V_{1}^{n}=v_{1}^{n}\left(S_{t_{1}}\right)$ and $V_{0}^{n}=v_{0}^{n}\left(s_{0}\right)$, etc. The quadratic quantization $\widehat{S}_{t_{1}}$ of $S_{t_{1}}$ being optimal and $S_{0}$ being deterministic, one has $\mathbb{E}\left(\Delta S_{t_{1}} / \Delta \widehat{S}_{t_{1}}\right)=\Delta \widehat{S}_{t_{1}}$. Then a straightforward computation shows that

$$
\begin{aligned}
& \mathbb{E}\left(\Delta S_{t_{1}} \Delta S_{t_{1}}^{*}\right)-\mathbb{E}\left(\Delta \widehat{S}_{t_{1}} \Delta \widehat{S}_{t_{1}}^{*}\right)=\mathbb{E}\left(\left(\Delta S_{t_{1}}-\Delta \widehat{S}_{t_{1}}\right)\left(\Delta S_{t_{1}}-\Delta \widehat{S}_{t_{1}}\right)^{*}\right) \\
& \text { so that } \quad\left\|\mathbb{E}\left(\Delta S_{t_{1}} \Delta S_{t_{1}}^{*}\right)-\mathbb{E}\left(\Delta \widehat{S}_{t_{1}} \Delta \widehat{S}_{t_{1}}^{*}\right)\right\| \leq \mathbb{E}\left\|\Delta S_{t_{1}}-\Delta \widehat{S}_{t_{1}}\right\|_{2}^{2} \leq\left(\frac{C}{N_{1}^{\frac{1}{d}}}\right)^{2} .
\end{aligned}
$$

\section{Now}

$$
\begin{aligned}
\mid \mathbb{E}\left(\left(V_{1}^{n}-V_{0}^{n}\right) \Delta S_{t_{1}}\right) & -\mathbb{E}\left(\left(\widehat{V}_{1}^{n}-\widehat{V}_{0}^{n}\right) \Delta \widehat{S}_{t_{1}}\right) \mid \\
& \leq\left\|\Delta \widehat{S}_{t_{1}}\right\|_{2}\left(\left\|V_{1}^{n}-\widehat{V}_{1}^{n}\right\|_{2}+\left|V_{0}^{n}-\widehat{V}_{0}^{n}\right|\right)+\left\|V_{1}^{n}\right\|_{2}\left\|S_{t_{1}}-\widehat{S}_{t_{1}}\right\|_{2} \\
& \leq\left\|\Delta S_{t_{1}}\right\|_{2} C\left(1+\left|s_{0}\right|\right) \frac{n}{(N / n)^{\frac{1}{d}}}+\frac{C}{N_{1}^{\frac{1}{d}}} \leq C\left(1+\left|s_{0}\right|\right) \frac{\sqrt{n}}{(N / n)^{\frac{1}{d}}}
\end{aligned}
$$

where we used in the last inequality that $\left\|\widehat{S}_{t_{1}}\right\|_{2} \leq\left\|S_{t_{1}}\right\|_{2} \leq C \sqrt{\frac{T}{n}}\left(1+\left|s_{0}\right|\right)$. One derives from (56) and (57) that

$$
\begin{aligned}
\left|\mathbb{E}\left(\Delta S_{t_{1}} \Delta S_{t_{1}}^{*}\right)\left(\zeta_{0}^{n}-\widehat{\zeta}_{0}^{n}\right)\right| \leq & \left|\mathbb{E}\left(\left(V_{1}^{n}-V_{0}^{n}\right) \Delta S_{t_{1}}\right)-\mathbb{E}\left(\left(\widehat{V}_{1}^{n}-\widehat{V}_{0}^{n}\right) \Delta \widehat{S}_{t_{1}}\right)\right| \\
& +\left\|\mathbb{E}\left(\Delta S_{t_{1}} \Delta S_{t_{1}}^{*}\right)-\mathbb{E}\left(\Delta \widehat{S}_{t_{1}} \Delta \widehat{S}_{t_{1}}^{*}\right)\right\|\left|\widehat{\zeta}_{0}\right| \\
\leq & C\left(1+\left|s_{0}\right|\right) \frac{\sqrt{n}}{(N / n)^{\frac{1}{d}}}+\frac{C}{N_{1}^{\frac{2}{d}}} \leq C\left(1+\left|s_{0}\right|\right) \frac{\sqrt{n}}{(N / n)^{\frac{1}{d}}} .
\end{aligned}
$$

Hence, one obtains the following result by inverting the covariance matrix since

$$
\left|\zeta_{0}^{n}-\widehat{\zeta}_{0}^{n}\right| \leq\left\|\left(\mathbb{E}\left(\Delta S_{t_{1}} \Delta S_{t_{1}}^{*}\right)\right)^{-1}\right\| C\left(1+\left|s_{0}\right|\right) \frac{\sqrt{n}}{(N / n)^{\frac{1}{d}}} .
$$

Now, it follows from the obvious $c c^{*}(x) \geq \varepsilon_{0} \operatorname{Diag}\left(x_{i}^{2}\right)$ that

$\mathbb{E}\left(\Delta S_{t_{1}} \Delta S_{t_{1}}^{*}\right) \geq\left(\varepsilon_{0} \int_{0}^{t_{1}} \min _{1 \leq i \leq d} \mathbb{E}\left(S_{s}^{i}\right)^{2} d s\right) I_{d} \geq\left(\varepsilon_{0} \int_{0}^{t_{1}} \min _{1 \leq i \leq d}\left(\mathbb{E} S_{s}^{i}\right)^{2} d s\right) I_{d}=\left(\min _{i}\left(s_{0}^{i}\right)^{2} \frac{\varepsilon_{0} T}{n}\right) I_{d}$

so that $\left.\| \mathbb{E}\left(\Delta S_{t_{1}} \Delta S_{t_{1}}^{*}\right)\right) \| \leq \varepsilon_{0}^{-2}\left(\min _{i} s_{0}^{i}\right)^{-2} \frac{n}{T}$ which completes the proof. $\diamond$ 


\subsection{Approximation of the strategy: rate of convergence}

In this section we evaluate the "global" residual risk on $[0, T-\delta]$ induced by the use of the time discretization of the diffusion with step $T / n$ i.e.

$$
\mathbb{E} \int_{0}^{T-\delta}\left|c^{*}\left(S_{s}\right)\left(Z_{s}-\zeta_{s}\right)\right|^{2} d s \quad \text { for some } \delta>n^{-1 / 3}
$$

where $Z_{t}$ is defined by (34) and $\zeta_{t}:=\operatorname{proj}_{n}(Z)$ is the projection on the set $\mathcal{P}_{n}$ of elementary predictable strategies. Our basic assumption is

$$
(\mathcal{H}) \equiv(i) \sigma \in C_{b}^{\infty}\left(\mathbb{R}^{d}\right), \quad(\text { ii }) \quad \sigma \sigma^{*} \geq \varepsilon_{0} I_{d}, \quad(i i i) \quad\|\nabla c\|_{\infty}<+\infty .
$$

Note that $\nabla c(x)=\partial \sigma(x) x+\sigma(x)$ where $\partial \sigma=\left(\partial \sigma_{1}, \ldots, \partial \sigma_{d}\right)$ with $\partial \sigma_{i}$ the Jacobian matrix of the $i^{\prime t h}$ column of the matrix $\sigma$. So $\nabla c$ is generally not bounded. However, if $\partial \sigma(x)=O\left(|x|^{-1}\right)$ when $|x|$ goes to infinity, then $\|\nabla c\|_{\infty}$ is finite.

Theorem 7 Assume that $(\mathcal{H})$ holds true. Let $\delta_{n}:=n^{-1 / 3}$. Then there exists some real constants $K$ and $\theta$ (depending on the bounds of $c$ and its first two derivatives) such that

$$
\mathbb{E} \int_{0}^{T-\delta_{n}}\left|c^{*}\left(S_{s}\right)\left(Z_{s}-\zeta_{s}\right)\right|^{2} d s \leq \frac{K\left(1+\left|s_{0}\right|\right)^{q}}{a} \frac{1}{n^{\frac{1}{6}-\frac{\theta}{\sqrt{\ln n}}}} .
$$

Remarks: Roughly speaking the above result says that on every $[0, T-\delta], \delta>0$, the speed of convergence in $L^{2}$ is of order $\frac{1}{n^{1 / 6}}$. Let us now comment the true statement.

- The fact that we may take $\left[0, T-\delta_{n}\right], \delta_{n}=n^{-1 / 3}$ says that asymptotically we control the whole interval $[0, T)$.

- The fact that $\frac{\theta}{\sqrt{\ln n}}$ comes out is due to the non uniform ellipticity of $S$ : this is the cost of truncation around zero. One may look at that some way round: if we had worked with the uniformly elliptic diffusion $X=\ln S$ instead of $S$, then the obstacle function becomes $h(t, \exp x)$ and has an exponential growth. So we need to truncate as well and the cost is still $\sqrt{\ln n}$.

- In most financial applications the obstacle $h$ is at most Lipschitz continuous (for example $h(t, x)=e^{-r t}\left(K-e^{r t} x\right)_{+}$for a put of strike $\left.K\right)$. However, if the obstacle is more regular, namely $h \in C^{1,2}$, then no regularize is needed and the resulting error is of order $1 / n^{1 / 3}$.

Some technical difficulties arise when evaluating the term in (58) directly, so we first reduce the problem to a simpler one. This is done in two steps.

Lemma 1 (STEP 1) Set $H_{s}:=c^{*}\left(S_{s}\right) Z_{s}$ and $\eta_{s}:=\frac{n}{T} \mathbb{E}_{t_{k}} \int_{t_{k}}^{t_{k+1}} H_{u} d u, s \in\left[t_{k}, t_{k+1}\right)$. Then

$$
\mathbb{E} \int_{0}^{T}\left|c^{*}\left(S_{s}\right)\left(Z_{s}-\zeta_{s}\right)\right|^{2} d s \leq \frac{C}{n}+\mathbb{E} \int_{0}^{T}\left|H_{s}-\eta_{s}\right|^{2} d s
$$


Proof: We temporarily define $z_{s}:=\frac{1}{t_{k+1}-t_{k}} \mathbb{E}_{t_{k}} \int_{t_{k}}^{t_{k+1}} Z_{r} d r, t_{k} \leq s<t_{k+1}$. Note that $z$ is an adapted process which is piecewise constant. Since $\zeta$ is the $L^{2}$-projection of $Z$ on the subspace of these type of processes, we have

$$
\begin{aligned}
\mathbb{E} \int_{0}^{T}\left|c^{*}\left(S_{s}\right)\left(Z_{s}-\zeta_{s}\right)\right|^{2} d s & \leq \mathbb{E} \int_{0}^{T}\left|c^{*}\left(S_{s}\right)\left(Z_{s}-z_{s}\right)\right|^{2} d s \\
& \leq 2 \mathbb{E} \int_{0}^{T}\left|H_{s}-\eta_{s}\right|^{2} d s+2 \mathbb{E} \int_{0}^{T}\left|\eta_{s}-c^{*}\left(S_{s}\right) z_{s}\right|^{2} d s .
\end{aligned}
$$

It remains to prove that the second term in the right hand of the above inequality is dominated by $C / n$. We write this term as

$$
\begin{aligned}
\mathbb{E} \sum_{k=0}^{n-1} \int_{t_{k}}^{t_{k+1}}\left|\frac{c^{*}\left(S_{s}\right)}{\Delta t_{k+1}} \mathbb{E}_{t_{k}} \int_{t_{k}}^{t_{k+1}} Z_{u} d u-\frac{1}{\Delta t_{k+1}} \mathbb{E}_{t_{k}} \int_{t_{k}}^{t_{k+1}} c^{*}\left(S_{u}\right) Z_{u} d u\right|^{2} d s \leq 2(I+J) \\
\text { with } I:=\mathbb{E} \sum_{k=0}^{n-1} \int_{t_{k}}^{t_{k+1}}\left|\frac{c^{*}\left(S_{s}\right)-c^{*}\left(S_{t_{k}}\right)}{\Delta t_{k+1}} \mathbb{E}_{t_{k}} \int_{t_{k}}^{t_{k+1}} Z_{u} d u\right|^{2} d s \\
J:=\mathbb{E} \sum_{k=0}^{n-1} \int_{t_{k}}^{t_{k+1}}\left|\frac{1}{\Delta t_{k+1}} \mathbb{E}_{t_{k}} \int_{t_{k}}^{t_{k+1}}\left(c^{*}\left(S_{u}\right)-c^{*}\left(S_{t_{k}}\right)\right) Z_{u} d u\right|^{2} d s .
\end{aligned}
$$

Let us evaluate $J$. Set $\underline{s}:=t_{k}$ if $s \in\left[t_{k}, t_{k+1}\right)$. Conditional Schwartz's inequality implies that

$$
\begin{aligned}
\left|\mathbb{E}_{t_{k}} \int_{t_{k}}^{t_{k+1}}\left(c^{*}\left(S_{u}\right)-c^{*}\left(S_{t_{k}}\right)\right) Z_{u} d u\right|^{2} & \leq \mathbb{E}_{t_{k}} \int_{t_{k}}^{t_{k+1}} \| c^{*}\left(S_{u}\right)-c^{*}\left(S_{t_{k}} \|^{2} d u \mathbb{E}_{t_{k}} \int_{t_{k}}^{t_{k+1}}\left|Z_{u}\right|^{2} d u\right. \\
& \leq\left[c^{*}\right]_{L i p}^{2} \int_{t_{k}}^{t_{k+1}} \mathbb{E}_{t_{k}}\left|S_{u}-S_{t_{k}}\right|^{2} d u \mathbb{E}_{t_{k}} \int_{t_{k}}^{t_{k+1}}\left|Z_{u}\right|^{2} d u .
\end{aligned}
$$

Now, classical results about Euler schemes of diffusions with Lipschitz continuous coefficients yield that, for every $u \in\left[t_{k}, t_{k+1}\right)$,

$$
\mathbb{E}_{t_{k}}\left|S_{u}-S_{t_{k}}\right|^{2} \leq C \Delta t_{k+1} \mathbb{E}_{t_{k}}\left(\left(1+\sup _{t \in[0, T]}\left|S_{t}\right|\right)^{2}\right) .
$$

for some positive real constant $C$. Consequently

$$
J \leq C \frac{T}{n} \mathbb{E}\left(\sum_{k=0}^{n-1} \mathbb{E}_{t_{k}}\left(\left(1+\sup _{t \in[0, T]}\left|S_{t}\right|\right)^{2}\right) \mathbb{E}_{t_{k}} \int_{t_{k}}^{t_{k+1}}\left|Z_{u}\right|^{2} d u\right)
$$

INRIA 


$$
\begin{aligned}
& \leq C \frac{T}{n} \mathbb{E}\left(\left(1+\sup _{t \in[0, T]}\left|S_{t}\right|\right)^{2} \sum_{k=0}^{n-1} \mathbb{E}_{t_{k}} \int_{t_{k}}^{t_{k+1}}\left|Z_{u}\right|^{2} d u\right) \\
& \leq \frac{C}{n}\left\|\left(1+\sup _{t \in[0, T]}\left|S_{t}\right|\right)^{2}\right\|_{2}\left\|\sum_{k=0}^{n-1} \mathbb{E}_{t_{k}} \lambda_{k+1}\right\|_{2}
\end{aligned}
$$

where $\lambda_{k+1}:=\int_{t_{k}}^{t_{k+1}}\left|Z_{u}\right|^{2} d u$ for every $k \in\{1, \ldots, n-1\}$. Since the $\lambda_{k}$ 's are nonnegative,

$$
\sum_{k=0}^{n-1} \lambda_{k+1}^{2} \leq\left(\sum_{k=0}^{n-1} \lambda_{k+1}\right)^{2}
$$

so that $\mathbb{E}\left(\sum_{k=0}^{n-1} \mathbb{E}_{t_{k}} \lambda_{k+1}\right)^{2} \leq 2 \mathbb{E}\left(\sum_{k=0}^{n-1}\left(\lambda_{k+1}-\mathbb{E}_{t_{k}} \lambda_{k+1}\right)\right)^{2}+2 \mathbb{E}\left(\sum_{k=0}^{n-1} \lambda_{k+1}\right)^{2}$

$$
\begin{aligned}
& \leq 2 \mathbb{E} \sum_{k=0}^{n-1}\left(\lambda_{k+1}-\mathbb{E}_{t_{k}} \lambda_{k+1}\right)^{2}+2 \mathbb{E}\left(\sum_{k=0}^{n-1} \lambda_{k+1}\right)^{2} \\
& \leq 4 \mathbb{E}\left(\sum_{k=0}^{n-1} \lambda_{k+1}\right)^{2}=4 \mathbb{E}\left(\int_{0}^{T}\left|Z_{u}\right|^{2} d u\right)^{2} .
\end{aligned}
$$

Finally

$$
J \leq \frac{C}{n}\left\|\left(1+\sup _{t \in[0, T]}\left|S_{t}\right|\right)^{2}\right\|_{2}\left\|\int_{0}^{T}\left|Z_{u}\right|^{2} d u\right\|_{2} .
$$

It is a standard result on diffusions that $\left\|\left(1+\sup _{t \in[0, T]}\left|S_{t}\right|\right)^{2}\right\|_{2}$ is finite. It remains to prove that the term involving $Z$ is finite. Since $c c^{*}\left(S_{s}\right) \geq \varepsilon_{0} S_{s} S_{s}^{*} I_{d}$, it follows that $\left|Z_{s}\right|^{2} \leq$ $\varepsilon_{0}^{-1} \max _{1 \leq i \leq d}\left(S_{s}^{i}\right)^{2}\left|H_{s}\right|^{2}$ so that, Schwartz Inequality yields

$\mathbb{E}\left(\int_{0}^{T}\left|Z_{s}\right|^{2} d s\right)^{2} \leq\left(\mathbb{E} \sup _{0 \leq t \leq T}\left|\left(S_{t}^{i}\right)^{-1}\right|^{8}\right)^{1 / 2}\left(\mathbb{E}\left(\int_{0}^{T}\left|H_{s}\right|^{2} d s\right)^{4}\right)^{1 / 2} \leq C\left(\mathbb{E}\left(\int_{0}^{T}\left|H_{s}\right|^{2} d s\right)^{4}\right)^{1 / 2}<+\infty$.

As $S_{t}^{-1}:=\left(1 / S_{t}^{i}\right)$ satisfies an equation similar to (1), its supremum has finite polynomial moments. Finally, the last inequality is a standard fact from $R B S D E$ theory (see [20] or [2]). So we have proved that $J \leq C / n$.

One treats $I$ the same way round. $\diamond$

STEP 2 The second type of difficulty which appears is due to the following two facts:

- The obstacle $h\left(t, S_{t}\right)$ is not sufficiently smooth and so we do not have a nice control on the increasing process $K$. 
- The diffusion process $S$ is not uniformly elliptic (because $c(0)=0$ ) and so we do not have nice evaluations of the density of $S_{t}$.

In order to overcome these difficulties we will replace $S$ by an elliptic diffusion denoted $\underline{S}$ and the obstacle $h$ by a smooth obstacle $\underline{h}$. Namely, let $\varepsilon>0$ and $\lambda>0$. We define:

- A function $\underline{h} \in C^{1,2}\left(\mathbb{R}_{+} \times \mathbb{R}^{d}, \mathbb{R}\right)$ using a regularization by convolution of order $\varepsilon$ of $h$. In particular, since $h$ is Lipschitz continuous, we have

$$
\|h-\underline{h}\|_{\infty} \leq C \varepsilon \quad \text { and } \quad\left\|\left(\partial_{t}+\mathcal{L}_{c}\right) \underline{h}\right\|_{\infty} \leq C \varepsilon^{-1}
$$

where $\mathcal{L}_{c}$ is the infinitesimal generator of the diffusion $S$.

- A function $\varphi_{\lambda} \in C_{b}^{\infty}\left(\mathbb{R}^{d}, \mathbb{R}^{d}\right)$ satisfying

$$
\varphi_{\lambda}(x):=\left\{\begin{array}{ll}
x & \text { if }|x| \geq e^{-\lambda} \\
\frac{x}{2|x|} e^{-\lambda} & \text { if }|x| \leq \frac{1}{2} e^{-\lambda}
\end{array} \text { and } \sup _{\lambda>0}\left\|D^{\alpha} \varphi_{\lambda}\right\|_{\infty} \leq C_{\alpha} \text { for every multi-index } \alpha .\right.
$$

Then the approximating diffusion coefficient $c_{\lambda}:=c \circ \varphi_{\lambda}$ satisfies

$$
c_{\lambda} c_{\lambda}^{*}(x) \geq \frac{\varepsilon_{0}}{4} e^{-2 \lambda} \quad \text { and } \quad\left\|D^{\alpha} c_{\lambda}\right\|_{\infty} \leq C_{\alpha} \quad \text { for every } \alpha .
$$

We consider now the solution $\underline{S}^{x}$ of the $S D E$

$$
d \underline{S}_{t}=\underline{S}_{t}\left(r d t+c_{\lambda}\left(\underline{S}_{t}\right) d W_{t}\right), \quad \underline{S}_{0}=x .
$$

Sometimes $\underline{S}_{t}^{x}$ will denote the solution starting at $x$. The related Snell envelope

$$
\underline{Y}_{t}=\operatorname{ess} \sup _{\tau \in \mathcal{T}_{t}} \mathbb{E}_{t} \underline{h}\left(\tau, \underline{S}_{\tau}\right)
$$

satisfies the $R B S D E$

$$
\underline{Y}_{t}=\underline{h}\left(T, \underline{S}_{T}\right)+\underline{K}_{T}-\underline{K}_{t}-\int_{t}^{T} \underline{H}_{s} \cdot d W_{s}
$$

for some non decreasing process $\underline{K}$ and some adapted square integrable process $\underline{H}$. We refer to [20] and [2] for this topic. We also consider the approximation

$$
\underline{\eta}_{s}=\frac{n}{T} \mathbb{E}_{t_{k}} \int_{t_{k}}^{t_{k+1}} \underline{H}_{s} d s, t_{k} \leq s<t_{k+1}
$$

Then we have the following lemma.

Lemma 2 Assume that $(\mathcal{H})$ holds

$$
\mathbb{E} \int_{0}^{T}\left|H_{s}-\eta_{s}\right|^{2} d s \leq C\left(e^{-C \lambda^{2} / T}+\varepsilon^{2}\right)+\mathbb{E} \int_{0}^{T}\left|\underline{H}_{s}-\underline{\eta}_{s}\right|^{2} d s
$$


Proof: We use the stability property of $R B S D E^{\prime}$ s (see [20] and [2]) in order to obtain

$$
\begin{aligned}
\mathbb{E} \int_{0}^{T}\left|H_{s}-\underline{H}_{s}\right|^{2} d s & \leq C \mathbb{E} \sup _{0 \leq s \leq T}\left|h\left(s, S_{s}\right)-\underline{h}\left(s, \underline{S}_{s}\right)\right|^{2} \\
& \leq C\left(\varepsilon^{2}+\mathbb{E} \sup _{0 \leq s \leq T}\left|h\left(s, S_{s}\right)-h\left(s, \underline{S}_{s}\right)\right|^{2}\right) .
\end{aligned}
$$

Define $\tau:=\inf \left\{t /\left|S_{t}\right| \leq e^{-\lambda}\right\}$ and we note that

$$
\mathbb{P}(\tau \leq T)=\mathbb{P}\left(\inf _{0 \leq s \leq T}\left|S_{s}\right| \leq e^{-\lambda}\right)=\mathbb{P}\left(\sup _{0 \leq s \leq T}\left|\log S_{s}\right| \geq \lambda\right) \leq C e^{-C \lambda^{2} / T}
$$

the last inequality is a standard large deviation fact (see e.g. [27] although it can be easily checked directly on model (1).

Since $S_{t}=\underline{S}_{t}$ for $t \leq \tau$, we obtain

$$
\begin{aligned}
\mathbb{E} \int_{0}^{T}\left|H_{s}-\underline{H}_{s}\right|^{2} d s & \leq C\left(\varepsilon^{2}+\mathbb{E}\left(\sup _{0 \leq s \leq T}\left(\left|h\left(s, S_{s}\right)\right|^{2}+\left|h\left(s, \underline{S}_{s}\right)\right|^{2}\right) \mathbf{1}_{\{\tau \leq T\}}\right)\right) \\
& \leq C\left(\varepsilon^{2}+e^{-C \lambda^{2} / T}\right) .
\end{aligned}
$$

On the other hand since $\eta$ and $\eta$ are the $L^{2}(d t d \mathbb{P})$-projections of $H$ and $\underline{H}$ respectively on the space $\mathcal{P}_{n}$ of elementary predictable processes, we have

$$
\mathbb{E} \int_{0}^{T}\left|\eta_{s}-\underline{\eta}_{s}\right|^{2} d s \leq \mathbb{E} \int_{0}^{T}\left|H_{s}-\underline{H}_{s}\right|^{2} d s \leq C\left(\varepsilon^{2}+e^{-C \lambda^{2} / T}\right)
$$

and the proof is completed. $\diamond$

We need now some analytical facts that we recall here (see [20] and [2]). First of all we have the representation

$$
\underline{Y}_{t}=u\left(t, \underline{S}_{t}\right), \quad \underline{H}_{t}=\left(c_{\lambda}^{*} \nabla u\right)\left(t, \underline{S}_{t}\right)
$$

where $u$ is the unique (in some sense not important here, see [2]) solution of the $P D E$

$$
\left(\partial_{t}+\mathcal{L}_{c}\right) u(t, x)+\underline{F}(t, x, u(t, x))=0, \quad u(T, x)=\underline{h}(T, x),
$$

with

$$
\underline{F}(t, x, u(t, x))=\alpha(t, x) 1_{\{u(t, x)=\underline{h}(t, x)\}}\left(\left(\partial_{t}+\mathcal{L}_{c}\right) \underline{h}(t, x)\right)_{+}
$$

where $\alpha$ is a measurable function such that $0 \leq \alpha \leq 1$. Denote

$$
F(t, x)=\underline{F}(t, x, u(t, x))
$$

and notice (recall (61)) that

$$
\sup _{0 \leq t \leq T} \sup _{x \in \mathbb{R}^{d}}|F(t, x)| \leq \frac{C}{\varepsilon}
$$

$\mathrm{RR} \mathrm{n}^{\circ} 4465$ 
With this notation $u$ satisfies

$$
\left(\partial_{t}+\mathcal{L}_{c}\right) u(t, x)+F(t, x)=0, \quad u(T, x)=\underline{h}(T, x),
$$

and consequently $u$ satisfies the mild form of the above $P D E$

$$
u(t, x)=\underline{P}_{T-t} \underline{h}_{T}(x)+\int_{t}^{T} \underline{P}_{s-t} F_{s}(x) d s
$$

where $\left(\underline{P}_{t}\right)_{t \geq 0}$ is the semi-group of the diffusion $\underline{S}_{t}$, that is $\underline{P}_{t} f(x)=\mathbb{E} f\left(\underline{S}_{t}^{x}\right)$. This is the equation that will be used in the sequel.

We turn now to the semi-group. It is well known (see [24] or [31]) that under the hypothesis $(62), \underline{P}_{t}(x, y)=p_{t}(x, y) d y$ and for every $k \in \mathbb{N}$ and every multi-index $\alpha=$ $\left(\alpha_{1}, \ldots, \alpha_{m}\right)$ we have

$$
\left|\partial_{t}^{k} D_{x}^{\alpha} p_{t}(x, y)\right| \leq \frac{K(1+|x|)^{q}}{a t^{k+\frac{m+1}{2}}} e^{2 \lambda} \times e^{-K^{\prime} \frac{|x-y|^{2}}{t}}
$$

where $K, K^{\prime}, q$ depend on $\alpha$ and on $C_{\alpha}$ from (62) (but not on $\lambda$ ). Let us pint out some immediate consequences of this evaluation in our framework. Since $\left|\underline{h}_{T}(y)\right| \leq C(1+|y|)$, using (65) we obtain

$$
\begin{aligned}
\left|\frac{\partial \underline{P}_{\delta} \underline{h}_{T}}{\partial x_{k}}(x)\right| & \leq \int_{\mathbb{R}^{d}}\left|\frac{\partial p_{\delta}(x, y)}{\partial x_{k}}\right| \times C(1+|y|) d y \leq \frac{1}{\sqrt{\delta}} \frac{K(1+|x|)^{q}}{a} e^{2 \lambda} \\
\left|\frac{\partial^{2} \underline{P}_{\delta} \underline{h}_{T}}{\partial x_{k} \partial x_{p}}(x)\right| \leq \frac{1}{\delta} \frac{K(1+|x|)^{q}}{a} e^{2 \lambda} & \leq \frac{\partial}{\partial x_{i}} \underline{P}_{T-t} \underline{h}_{T}(x)-\frac{\partial}{\partial x_{i}} \underline{P}_{T-s} \underline{h}_{T}(y) \mid \leq \frac{K(1+|x|+|y|)^{q}}{a \delta^{3 / 2}} e^{2 \lambda}(\sqrt{t-s}+|x-y|) .
\end{aligned}
$$

We deal now with the second term in the right hand of (72). Since $\|F\|_{\infty} \leq C / \varepsilon$, the same computations as above (using (65)) give

$$
\begin{aligned}
& \left|\frac{\partial \underline{P}_{\delta} F_{s}}{\partial x_{k}}(x)\right|+\left|\frac{\partial^{2} \underline{\underline{P}}_{\delta} F_{s}}{\partial x_{k} \partial x_{p}}(x)\right| \leq \frac{1}{\delta \varepsilon} \frac{K(1+|x|)^{q}}{a} e^{2 \lambda} \\
& \text { and } \\
& \left|\frac{\partial^{2} \underline{\underline{P}}_{\delta} F_{s}}{\partial s \partial x_{k}}(x)\right| \leq \frac{1}{\delta^{3 / 2} \varepsilon} \frac{K(1+|x|)^{q}}{a} e^{2 \lambda} .
\end{aligned}
$$

Lemma 3 Let $v_{i}=\frac{\partial u}{\partial x_{i}}$. Under the hypothesis $(\mathcal{H})$ (and consequently under (62)) one has

$$
\begin{aligned}
\left|v_{i}(t, x)-v_{i}(t, y)\right| & \leq \frac{K(1+|x|+|y|)^{q}}{a} e^{2 \lambda} \times\left(\frac{\sqrt{\delta}}{\varepsilon}+\frac{1}{\delta \varepsilon}|x-y|\right) \\
\text { and } & \\
\left|v_{i}(t, x)-v_{i}(s, x)\right| & \leq \frac{K(1+|x|)^{q}}{a} e^{2 \lambda} \times\left(\frac{\sqrt{\delta}}{\varepsilon}+\frac{1}{\delta \varepsilon} \sqrt{t-s}\right) .
\end{aligned}
$$


for every $x, y \in \mathbb{R}^{d}$ and every $t, s \geq 0$ such that $|t-s| \leq \delta$.

Proof: We take derivatives in the mild equation for $u$ and we obtain, for $t \leq T-\delta$

$$
\begin{aligned}
v_{i}(t, x) & =\frac{\partial}{\partial x_{i}} \underline{P}_{T-t} \underline{h}_{T}(x)+\int_{t}^{T} \frac{\partial}{\partial x_{i}} \underline{P}_{s-t} F_{s}(x) d s \\
& =\frac{\partial}{\partial x_{i}} \underline{P}_{T-(t+\delta)} \underline{P}_{\delta} \underline{h}_{T}(x)+\int_{t+\partial}^{T} \frac{\partial}{\partial x_{i}} \underline{P}_{s-(t+\delta)} \underline{P}_{\delta} F_{s}(x) d s+\int_{t}^{t+\delta} \frac{\partial}{\partial x_{i}} \underline{P}_{s-t} F_{s}(x) d s .
\end{aligned}
$$

Note that in the first two terms in the above (72) involve $\underline{P}_{\delta} F$, so one can use the regularization effect of the semi-group which is not the case for the third term. We evaluate first the last term in the right hand of the above equality. Using (65)

$$
\begin{aligned}
\left|\int_{t}^{t+\delta} \frac{\partial}{\partial x_{i}} \underline{P}_{s-t} F_{s}(x) d s\right| & \leq\|F\|_{\infty} \int_{t}^{t+\delta} \int_{\mathbb{R}^{d}}\left|\frac{\partial}{\partial x_{i}} p_{s-t}(x, y)\right| d y d s \\
& \leq\|F\|_{\infty} \frac{K(1+|x|)^{q}}{a} e^{2 \lambda} \int_{t}^{t+\delta} \int_{\mathbb{R}^{d}} \frac{1}{s-t} e^{-K^{\prime} \frac{|x-y|^{2}}{s-t}} d y d s \\
& \leq\|F\|_{\infty} \frac{K(1+|x|)^{q}}{a} e^{2 \lambda} \int_{t}^{t+\delta} \frac{1}{\sqrt{s-t}} d s \\
& \leq \frac{\sqrt{\delta}}{\varepsilon} \frac{K(1+|x|)^{q}}{a} e^{2 \lambda}
\end{aligned}
$$

the last inequality being a consequence of $\|F\|_{\infty} \leq C / \varepsilon$. We deal now with the first term in the $R H S$ of (72). Using the Feynmann-Kac formula

$$
\frac{\partial}{\partial x_{i}} \underline{P}_{T-(t+\delta)} \underline{P}_{\delta} \underline{h}_{T}(x)=\frac{\partial}{\partial x_{i}} \mathbb{E} \underline{P}_{\delta} \underline{h}_{T}\left(\underline{S}_{T-(t+\delta)}^{x}\right)=\sum_{k=1}^{d} \mathbb{E}\left(\frac{\partial \underline{P}_{\delta} \underline{h}_{T}}{\partial x_{k}}\left(\underline{S}_{T-(t+\delta)}^{x}\right) \frac{\partial \underline{S}_{T-(t+\delta)}^{x, k}}{\partial x_{i}}\right) .
$$

Using inequalities (66), (67) and (68), one checks that

$$
\begin{aligned}
\mid \mathbb{E}\left(\frac{\partial \underline{P}_{\delta} \underline{h}_{T}}{\partial x_{k}}\left(\underline{S}_{T-(t+\delta)}^{x}\right) \frac{\partial \underline{S}_{T-(t+\delta)}^{x, k}}{\partial x_{i}}\right. & \left.-\frac{\partial \underline{P}_{\delta} \underline{h}_{T}}{\partial x_{k}}\left(\underline{S}_{T-\left(t^{\prime}+\delta\right)}^{y}\right) \frac{\partial \underline{S}_{T-\left(t^{\prime}+\delta\right)}^{y, k}}{\partial x_{i}}\right) \mid \\
& \leq \frac{1}{\delta} \frac{K(1+|x|+|y|)^{q}}{a} e^{2 \lambda}\left(|x-y|+\sqrt{t-t^{\prime}}\right) .
\end{aligned}
$$

We turn now to the second term in the right hand of (72). Using (69), one obtains

$$
\int_{t+\delta}^{T}\left|\frac{\partial}{\partial x_{i}} \underline{P}_{s-(t+\delta)} \underline{P}_{\delta} F_{s}(x)-\frac{\partial}{\partial x_{i}} \underline{P}_{s-(t+\delta)} \underline{P}_{\delta} F_{s}(y)\right| \leq \frac{K(1+|x|+|y|)^{q}}{a \delta \varepsilon} e^{2 \lambda}|x-y| .
$$

Consider now $t^{\prime}>t$ and write

$$
\left|\int_{t+\delta}^{T} \frac{\partial}{\partial x_{i}} \underline{P}_{s-t} F_{s}(x) d s-\int_{t^{\prime}+\delta}^{T} \frac{\partial}{\partial x_{i}} \underline{P}_{s-t^{\prime}} F_{s}(x) d s\right|
$$

$\mathrm{RR} \mathrm{n}^{\circ} 4465$ 


$$
\leq \int_{t+\delta}^{t^{\prime}+\delta}\left|\frac{\partial}{\partial x_{i}} \underline{P}_{s-t} F_{s}(x)\right| d s+\int_{t^{\prime}+\delta}^{T}\left|\frac{\partial}{\partial x_{i}} \underline{P}_{s-t} F_{s}(x) d s-\frac{\partial}{\partial x_{i}} \underline{P}_{s-t^{\prime}} F_{s}(x)\right| d s=: I+J
$$

Using (69) and (70), we obtain $I \leq \frac{K(1+|x|)^{q}}{a \delta \varepsilon} e^{2 \lambda}\left|t-t^{\prime}\right|$

$$
J \leq \frac{K(1+|x|)^{q}}{a \delta^{3 / 2} \varepsilon} e^{2 \lambda}\left|t-t^{\prime}\right| \leq \frac{K(1+|x|)^{q}}{a \delta \varepsilon} e^{2 \lambda} \sqrt{\left|t-t^{\prime}\right|}
$$

the last inequality being a consequence of $\left|t-t^{\prime}\right| \leq \delta$. This completes the proof . $\diamond$

The above lemma and the representation $\underline{H}_{t}^{x}=\left(c_{\lambda}^{*} \nabla u\right)\left(t, \underline{S}_{t}^{x}\right)$ straightforwardly yield

Corollary 1 For every $s<r<T-\delta$ such that $r-s<1 / n<\delta$,

$$
\left(\mathbb{E}\left|\underline{H}_{r}^{x}-\underline{H}_{s}^{x}\right|^{2}\right)^{1 / 2} \leq \frac{K(1+|x|)^{q}}{a} e^{2 \lambda} \times\left(\frac{\sqrt{\delta}}{\varepsilon}+\frac{1}{\delta \varepsilon} \frac{1}{\sqrt{n}}\right) .
$$

We are now able to prove Theorem 7.

Proof of Theorem 7: Using (73)

$$
\begin{aligned}
\mathbb{E} \int_{0}^{T-\delta}\left|\underline{H}_{s}-\underline{\eta}_{s}\right|^{2} d s & =\sum_{t_{k}<T-\delta} \mathbb{E} \int_{t_{k}}^{t_{k+1}}\left|\frac{1}{\Delta t_{k+1}} \int_{t_{k}}^{t_{k+1}}\left(\underline{H}_{s}-\underline{H}_{r}\right) d r\right|^{2} d s \\
& \leq \sum_{t_{k}<T-\delta} \int_{t_{k}}^{t_{k+1}} \frac{1}{\Delta t_{k+1}} \int_{t_{k}}^{t_{k+1}} \mathbb{E}\left|\underline{H}_{s}-\underline{H}_{r}\right|^{2} d r d s \\
& \leq \frac{K(1+|x|)^{2 q}}{a^{2}} e^{4 \lambda} \times\left(\frac{\sqrt{\delta}}{\varepsilon}+\frac{1}{\delta \varepsilon} \frac{1}{\sqrt{n}}\right)^{2} .
\end{aligned}
$$

Moreover, as a consequence of the first two lemmas

$\mathbb{E} \int_{0}^{T-\delta}\left|c^{*}\left(S_{s}\right)\left(Z_{s}-\zeta_{s}\right)\right|^{2} d s \leq \frac{C}{n}+C\left(e^{-C^{\prime} \lambda^{2} / T}+\varepsilon^{2}\right)+\frac{K(1+|x|)^{2 q}}{a^{2}} e^{4 \lambda} \times \frac{1}{\varepsilon^{2}}\left(\delta+\frac{1}{n \delta^{2}}\right)$.

In order to minimize $\delta+\frac{1}{n \delta^{2}}$ we take $\delta_{n}=n^{-1 / 3}$ so that $\delta+\frac{1}{n \delta^{2}}=C n^{-1 / 3}$. Then, in order to minimize $\varepsilon^{2}+\varepsilon^{-2} n^{-1 / 3}$ we take $\varepsilon_{n}=n^{-1 / 6}$ so that $\varepsilon^{2}+\varepsilon^{-2} n^{-1 / 3} \sim n^{-1 / 6}$. Finally we take $\lambda_{n}=\sqrt{\ln n}$ and to obtain (59). $\diamond$

\section{Numerical experiments on American exchange style options}

In this section, we present some numerical experiments concerning the pricing and the hedging of American style options in dimensions $d=2$ up to 10. This study will be divided

INRIA 
into two parts. First, we will specify the spatial accuracy in each dimension in order to be able to produce a good choice of time and space discretization. Secondly, we will compute some prices and hedges according to our previous "optimal" discretization.

\subsection{The model}

Due to the numerical cost of theses simulations (especially in large dimension), we will emphasize on model independent (with respect to the dividends rate, volatilities, ...) computations. For this reason the underlying assets $\left(S_{t}\right)_{t \in[0, T]}$ in $\mathbb{R}^{d}$ are null correlated and follows here the standard Black and Scholes models

$$
\mathrm{d} S_{t}^{i}=-\mu_{i} S_{t}^{i} \mathrm{~d} t+\sigma_{i} \mathrm{~d} W_{t}^{i}, \quad t \in[0, T], \quad i=1, \ldots, d,
$$

where $\mu_{i}>0$ are divided rates, $\sigma_{i}>0$ are the (constant) volatilities and $\left(W_{t}\right)_{t \in[0, T]}$ denotes a $d$-dimensional standard Brownian motion.

This choice is motivated by the importance of this model for applications. Furthermore, $S_{t}$ is a closed function of $\left(t, W_{t}\right)$ for every $t \in[0, T]$, namely $S_{t}^{i}=s_{0}^{i} \exp \left(-\left(\mu_{i}+\sigma_{i}^{2} / 2\right) t+\sigma_{i} W_{t}^{i}\right)$. Therefore the computation of the quantization tree of $\left(S_{t}\right)$ relies entirely on the one of $\left(W_{t}\right)$. In its turn, because of the null correlation assumption, such quantization is computed from the optimal quantization of the normal law in $\mathbb{R}^{d}$ and from the edge weights of Proposition 1. We point out that these computations are done once for all.

Concerning the contract, we focus here on American exchange style options with pay-offs

$$
h(x)=\max \left(x_{1} \cdots x_{p}-x_{p+1} \cdots x_{2 p}, 0\right) \quad(\text { set } d:=2 p) .
$$

We know that a fair price $\mathcal{V}_{t}$ at time $t$ and maturity $T$ of such a contract is given by the Snell envelope of the process $\left(h\left(S_{t}\right)\right)_{t \in[0, T]}$, namely

$$
e^{-r t} \mathcal{V}_{t}=\sup \left\{\mathbb{E}\left(e^{-r \tau} h\left(S_{\tau}\right) \mid \mathcal{F}_{t}\right), \quad \tau \in[t, T], \quad \tau \text { stopping time }\right\}
$$

where the filtration $\left(\mathcal{F}_{t}\right)_{t \in[0, T]}$ are those of the Brownian motion $\left(W_{t}\right)$.

In the framework of exchange options, we can show that such a price is independent of the interest rate $r$, it is why we will consider in this section that $r=0$.

It is important to note that there exists a closed form for the Black \& Scholes price of a European contract for (74)-(75) at time $t$ and maturity $T$ given by

$$
B S(\theta, x, y, \widetilde{\sigma}, r)=\operatorname{erf}\left(d_{1}\right) \exp (r \theta) x-\operatorname{erf}\left(d_{1}-\widetilde{\sigma} \sqrt{\theta}\right) y
$$

where $\theta:=T-t, \widetilde{\sigma}:=\sqrt{\sum_{i=1}^{d} \sigma_{i}^{2}}, r:=\sum_{i=1}^{p} \mu_{i}-\sum_{i=p+1}^{d} \mu_{i}, x:=\prod_{i=1}^{p} S_{i}(t), \quad y=\prod_{i=p+1}^{d} S_{i}(t)$, $d_{1}:=\frac{\ln (x / y)+\left(\widetilde{\sigma}^{2} / 2+r\right) \theta}{\widetilde{\sigma} \sqrt{\theta}}$ and erf denotes the distribution function of the standard normal distribution. 


\subsection{The numerical scheme}

Let us precise now the numerical scheme that we will implement. As mentioned above, our approach to pricing consists first in quantizing the $d$-dim Brownian motion. More precisely, let $T>0$ and $n, N$ two integers; set $\Delta t:=\frac{T}{n}$ and $t_{k}:=k \Delta t$. Spatial discretization depends on the time $t_{k}$. Indeed, we use the partition

$$
N=N_{0}+N_{1}+N_{2}+\cdots+N_{n}
$$

given by (32), which assigns the number of points $N_{k}$ to te $k^{t h}$ time layer. Typically, $N_{0}=1<N_{1}<\cdots<N_{n}=\rho_{d} N$ where $\rho_{d}$ is a constant depending on $d$. The $N_{k}$ 's are chosen in order to make the smallest possible quantization error for the $d$-dim Brownian motion. Now, assume that for each $k \in\{0, \ldots, n\}$ the $N_{k}$-optimal quantizer of the normal distribution on $\mathbb{R}^{d}$ has been computed. We deduce the $N_{k}$-optimal quantizer $\left(x_{i}^{k}\right)_{i=1, \ldots, N_{k}}$ of $W_{t_{k}}$ by a simple $\sqrt{t_{k}}$-dilatation.

Finally, introducing an abstract family of control variate variables $\left(M_{i}^{k}\right)_{1 \leq k \leq n}$, the tested scheme can be written as a slight modification of Algorithm (20)

$$
\left\{\begin{array}{l}
v_{i}^{n}:=h_{i}^{n}-M_{i}^{n}, \quad i=1, \ldots, N_{n}, \\
v_{i}^{k}:=\max \left\{h_{i}^{k}-M_{i}^{k}, \sum_{j=1}^{N_{k+1}} \pi_{i, j}^{k} v_{j}^{k+1}\right\}, \quad i=1, \ldots, N_{k}, k=0, \ldots, n-1
\end{array}\right.
$$

where $h_{i}^{k}:=h\left(s_{i, 1}^{k}, \ldots, s_{i, d}^{k}\right)$ with $s_{i, \ell}^{k}:=s_{0, \ell} \exp \left(-\left(\mu_{\ell}+\frac{\sigma_{\ell}^{2}}{2}\right) k \Delta t+\sigma_{\ell} x_{i}^{k}\right), \ell=1, \ldots, d$ and where the weights $\pi_{i, j}^{k}$ are Monte-Carlo proxies of the theoretical weights

$$
\frac{\mathbb{P}\left(W_{t_{k+1}} \in C_{j}^{k+1}, W_{t_{k}} \in C_{i}^{k}\right)}{\mathbb{P}\left(W_{t_{k}} \in C_{i}^{k}\right)} .
$$

The price at point $x_{i}^{k}$ is given by

$$
p_{i}^{k}=v_{i}^{k}+M_{i}^{k}
$$

Following (47) the hedging $\delta_{i}^{k}$ at $x_{i}^{k}$ is computed by

$$
\delta_{i, \ell}^{k}:=\frac{\sum_{j=1}^{N_{k+1}} \pi_{i, j}^{k}\left(v_{j}^{k+1}-v_{i}^{k}\right)\left(s_{j, \ell}^{k+1}-s_{i, \ell}^{k}\right)}{\sum_{j=1}^{N_{k+1}} \pi_{i, j}^{k}\left(s_{j, \ell}^{k+1}-s_{i, \ell}^{k}\right)^{2}}, \quad \ell=1, \ldots, d .
$$


Numerical experiments show that the choice of the so-called "control variables" $M_{i}^{k}$ is very important in practice. Note that any "control variables" $M_{i}^{k}$ such that

$$
\sum_{j=1}^{N_{k+1}} \pi_{i, j}^{k} M_{j}^{k+1} \approx M_{i}^{k} \quad \text { up to the spatial discretization }
$$

can be chosen here. A efficient choice is here to take

$$
M_{i}^{k}=B S\left(T-t_{k}, \prod_{\ell=1}^{p} s_{i, \ell}^{k}, \prod_{\ell=p+1}^{d} s_{i, \ell}^{k}, \widetilde{\sigma}, r\right) .
$$

We have to note that there exists a natural choice of $\left\{M_{i}^{k}\right\}$ for which one has equality in (80). It consists in computing in a parallel way the European price approximation by

$$
M_{i}^{k}=\sum_{j=1}^{N_{k+1}} \pi_{i, j}^{k} M_{j}^{k+1}, \quad \text { with } M_{i}^{n}=h_{i}^{n},
$$

But it is not difficult to check that this choice is numerically equivalent to compute directly $\left(v^{k}\right)$ in $(77)$ with $M_{i}^{k}=0$. Numerical computations will be run with the choice (81).

\subsection{Accuracy, stability}

We will now estimate the rate of convergence of the numerical solution given by (77) towards a reference one. Theorem 5 gives the expected error terms both in time and in space. We recall that for a time discretization parameter $n$ (number of time layers) and space discretization parameter $\bar{N}=N / n$ (average number of points per layer), we have

$$
E(n, \bar{N})=\left|p_{0}^{0}-P_{t h}\right| \approx \frac{c_{1}}{n}+c_{2} \frac{n}{(\bar{N})^{\alpha}},
$$

where $p(n, \bar{N})$ (resp. $\left.P_{t h}\right)$ is the computed price (resp. theoretical price) at $t=0$ of american style options.

\subsubsection{The one dimensional case}

We begin by the case of the 1-dimension. The reference model will be the standard one dimensional Black \& Scholes

$$
\mathrm{d} S_{t}=S_{t}\left(r \mathrm{~d} t+\sigma \mathrm{d} W_{t}\right), \quad t \in[0, T], \quad S_{0}=36,
$$

where $r=0.06, \sigma=0.2$. The american contract will be an American put with the payoff function $x \mapsto \max (K-x, 0)$ with $K=40$. 
We will try to estimate the space convergence rate $\alpha$ in (82). In Table 1 , we have computed for $n$ becoming larger $(n \in\{50, \ldots, 100\})$ the error $E(n, \bar{N})$ for $\bar{N} \in\{25,37,50,75\}$. Numerically we observe that for these choices of $n$ the error is almost the spatial error

$$
E(n, \bar{N}) \approx c_{2} \frac{n}{(\bar{N})^{\alpha}}=C_{2} n .
$$

In the last lines of Table 1 , we have computed $C_{2}$ by means of linear regression. The last line represents the correlation term obtained. Once $C_{2}$ computed, we can evaluate the rate $\alpha$ between two values $\bar{N}_{1}$ and $\bar{N}_{2}$ by

$$
\alpha=\frac{\ln C_{2}\left(\bar{N}_{2}\right) / C_{2}\left(\bar{N}_{1}\right)}{\ln \left(\bar{N}_{1}\right) / \ln \left(\bar{N}_{2}\right)} .
$$

Values of $\alpha$ are reported in the last line of Table 1. We note that the computed value $\alpha$ is better than the expected value $(\alpha=1)$. In a linear case (European option case) we can show that it is implied by the regularizing properties of the semi-group of the diffusion. Indeed the numerical integration formula applied to $f(X)$ where $f$ admits a second derivative and $X$ is a square integrable random variable can be written as (see [38])

$$
\left|\mathbb{E} f(X)-\sum_{i=1}^{N} \pi_{i} f\left(x_{i}\right)-\sum_{i=1}^{N} f^{\prime}\left(x_{i}\right) \mathbb{E}\left(\left(X-x_{i}\right) \mathbf{1}_{C_{i}(x)}\right)\right| \leq \frac{1}{2} L\left(f^{\prime \prime}\right) D_{N}^{X, 2},
$$

where $D_{N}^{X, 2}$ is of the order of $N^{-2 / d}$. But the optimality of the grid makes the term

$\mathbb{E}\left(\left(X-x_{i}\right) \mathbf{1}_{C_{i}(x)}(X)\right)=\frac{\partial D_{N}^{X, 2}}{\partial x_{i}}$ vanishes. It seems difficult to show that this heuristic applies in the American option case although we observed it. This better rate of convergence is a strong argument showing that the optimal quantization is worthwhile.

\subsubsection{Higher dimensional case}

The determination of spatial order involves long runs (because of the smallness of $\Delta t$ ) with increasing average values of $\bar{N}$. From dimension 4 to 10 , the storage of the matrix $\left\{\pi_{i, j}^{k}\right\}$ for such discretization is costly and make the computations intractable. Nevertheless, the previous calculations in one and two dimension conclude to a spatial order of $2 / d$ when the grids are optimal. In fact, such optimality becomes harder and harder to get in high dimensions, it is why we can guess that spatial order will be between $1 / d$ (the "grid order") and $2 / d$.

Table 2 shows

\subsection{Numerical experiments}

We now present numerical simulations of Eq. (76) using (77), (78), (79), (81). The maximal horizon time $T_{\max }$ is chosen to be equal to 1 . For a given spatial discretization parameter $\bar{N}=N / n$, we will work with a time discretization $\Delta t=T_{\max } / n$ where $n=n(d, \bar{N})$ is 
given in Theorem 5 in order to ensure stability as shown above. Space discretization is then achieved by the optimal quantization of the Brownian motion in $\mathbb{R}^{d}$ on a space-time grid $\Gamma^{d}=\left\{\Gamma_{k}^{d}\right\}_{0 \leq k \leq n(d, \bar{N})}$.

In order to investigate the numerical influence of the free boundary, we will distinguish in each study an in-the-money case and an out-of-the-money case. Furthermore, our numerical price and hedging will be compared to a reference setting which is here the numerical scheme employed by [41] in the 2D exchange option. The model parameters and initial data of the reference scheme are given by Table 3 in the in-the-money case and Table 4 in the out-ofthe-money case.

In the following figures are displayed the computed prices and hedges at time $t=0$ together with the reference ones as a function of the maturity $T \in\left[0, T_{\max }\right]$.

We first investigate the pricing and hedging of (74)-(76) when $d=2$. The values of the dividends, volatilities and initial conditions are displayed in Table 3 and 4 . We see in Figs. 1-4 that our computations are well fitted with the reference ones in both cases (in and out the money).

In the in-the-money case, when the dimension increases, we can see that our price increases faster than the reference price when the maturity grows (see Figs. 5, 9, 13). The maximal error remains inside $5 \%$ in all the cases. The same phenomenon can be observed on the computed hedges (see Figs. 6, 10, 14).

In the other case (out-of-the-money), very different behaviour are observed on the prices. Indeed whatever the dimension (from 4 to 10) is, the prices seem to be well computed (Figs. $7,11,15)$.

Such a modification of behaviour happens too in Fig. 6.4. Here we show the implied residual risk in the computation of the hedging by least-squares method (see (48)) as a function of the maturity. We can see there that the values of this risk is bigger in the inthe-money case than in the out-of-the-money case. This may suggest an explanation of the antagonism of the Figs. 5/7, 9/11, 13/15. Indeed it seems that numerical incompleteness of the market ("residual risk") has a bigger effect on the price "in-the-money" than "out-of-themoney". 
Table 1: Estimation of the spatial rate of convergence $\alpha$ of (82) in the dimension 1 and 2 .

\begin{tabular}{|c|c|c|c|c|c|c|}
\hline \multirow[b]{2}{*}{$n$} & \multicolumn{4}{|c|}{$d=1$} & \multicolumn{2}{|c|}{$d=2$} \\
\hline & $E(n, 25)$ & $E(n, 37)$ & $E(n, 50)$ & $E(n, 75)$ & $E(n, 300)$ & $E(n, 600)$ \\
\hline 50 & 0.1871 & 0.094 & 0.0573 & 0.0312 & & \\
\hline 55 & 0.2074 & 0.1043 & 0.0634 & 0.0347 & & \\
\hline 60 & 0.2213 & 0.1079 & 0.0645 & 0.0328 & & \\
\hline 65 & 0.2423 & 0.1197 & 0.0707 & 0.0367 & & \\
\hline 70 & 0.2616 & 0.1302 & 0.0773 & 0.0408 & & \\
\hline 75 & 0.2822 & 0.1398 & 0.0836 & 0.0442 & & \\
\hline 80 & 0.2982 & 0.147 & 0.0862 & 0.0442 & & \\
\hline 85 & 0.3186 & 0.1568 & 0.0921 & 0.0474 & & \\
\hline 90 & 0.3387 & 0.167 & 0.0985 & 0.0509 & & \\
\hline 95 & 0.3555 & 0.1734 & 0.102 & 0.0515 & & \\
\hline 100 & 0.3753 & 0.1854 & 0.1085 & & & \\
\hline$r$ & 1.00 & 1.00 & 0.99 & 0.97 & & \\
\hline$C_{2}$ & $3.77(-3)$ & $1.82(-3)$ & $1.03(-3)$ & $4.79(-4)$ & & \\
\hline$\alpha$ & 1.87 & 1.90 & 1.91 & & & \\
\hline
\end{tabular}

Table 2: Estimation of the spatial rate of convergence $\alpha$ of (82) for dimensions $d=4,6,10$.

\begin{tabular}{c||c|c|c|c|c|c}
\multicolumn{1}{l|}{} & \multicolumn{2}{c|}{$d=4$} & \multicolumn{2}{c|}{$d=6$} & \multicolumn{2}{c}{$d=10$} \\
$n$ & $E(n, 500)$ & $E(n, 750)$ & $E(n, 500)$ & $E(n, 1000)$ & $E(n, 500)$ & $E(n, 1000)$ \\
\hline 75 & 0.046843 & & & & & \\
80 & 0.049243 & & & & & \\
85 & 0.053543 & & & & & \\
90 & 0.056643 & & & & & \\
95 & 0.060443 & & & & &
\end{tabular}

INRIA 


\begin{tabular}{c||c|c}
$\mathrm{i}$ & 1 & 2 \\
\hline \hline$\mu_{i}$ & $-5 \%$ & 0 \\
\hline$\sigma_{i}$ & $20 \%$ & $20 \%$ \\
\hline$s_{0, i}$ & 40 & 36
\end{tabular}

Table 3: Values of the parameter model when $d=2$ in the in-the-money case.

$$
d=2, \quad N_{\max }=300, \quad n=25
$$

In-the-money case

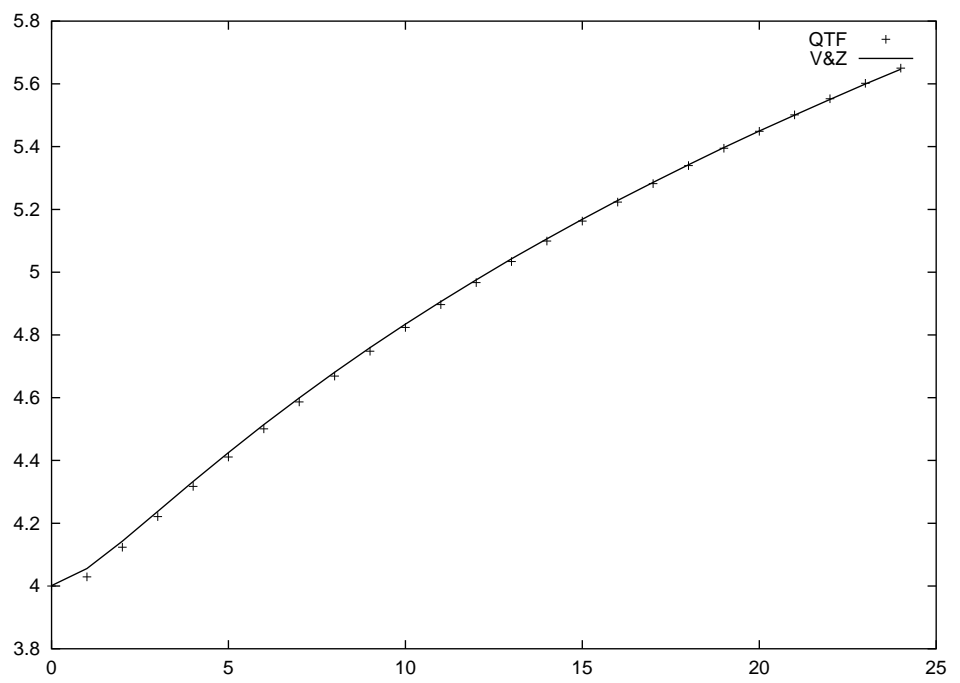

Figure 1: American exchange option price $\left(S_{1}-S_{2}\right)_{+}$function of the maturity where the number of layers is 25 and the number of points on the top is $300 .+$ depicts the price obtained with the method of quantization and - depicts the reference price (V \& Z) (cf. [41]).

$\mathrm{RR} \mathrm{n}^{\circ} 4465$ 
a)

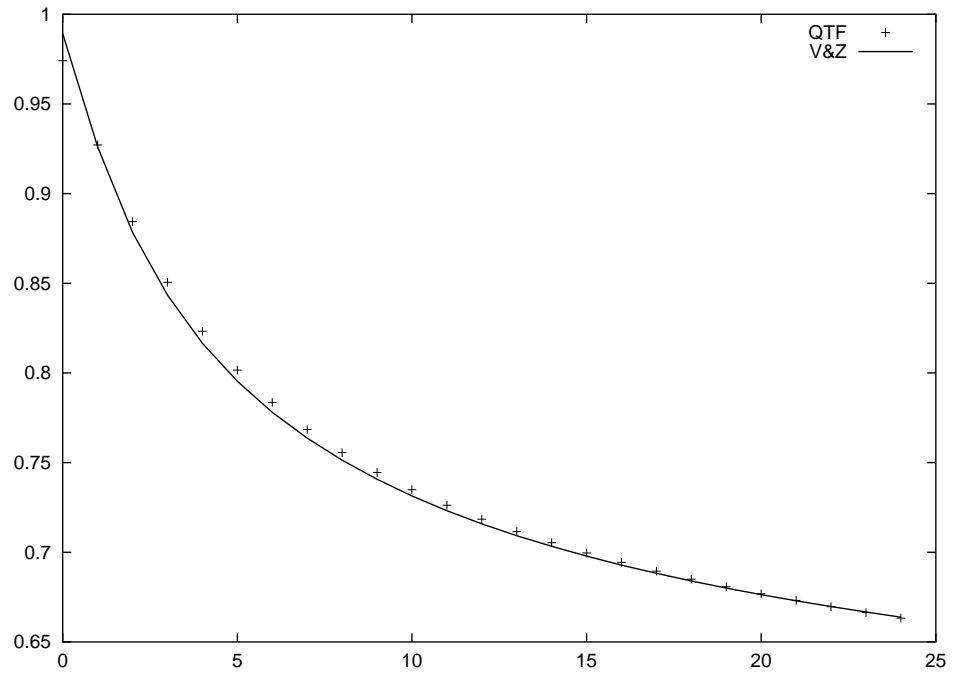

b)

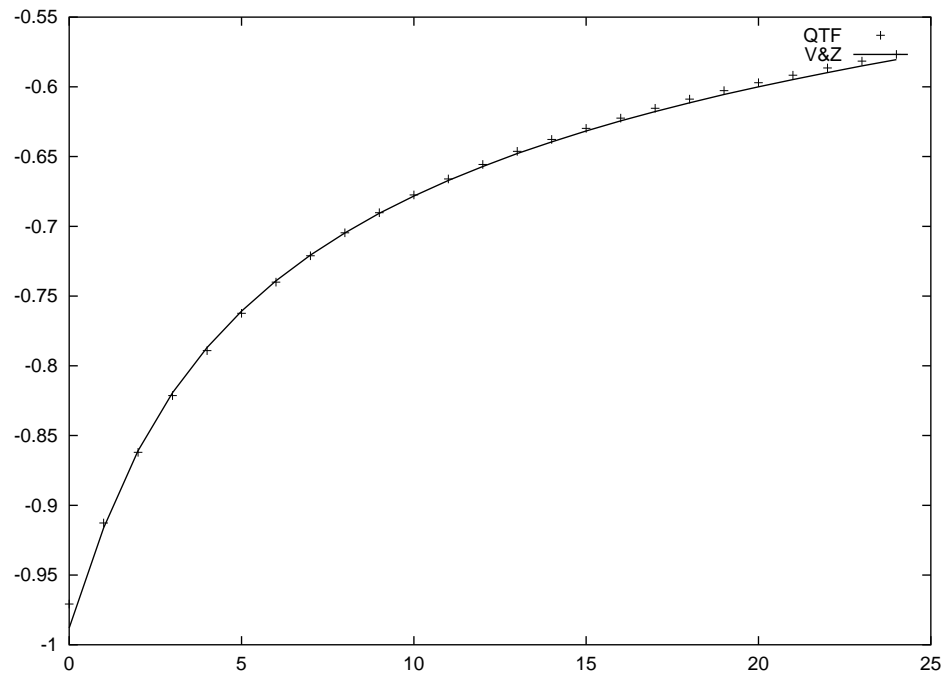

Figure 2: Hedging for the American option of Fig. 1. a) $\delta_{1}(+)$ fonction of the maturity. b) $\delta_{2}(+)$ fonction of the maturity. The reference price is denoted by - . 
Table 4: Value of the parameter model when $d=2$ in the out-of-the-money case.

\begin{tabular}{c||c|c}
$\mathrm{i}$ & 1 & 2 \\
\hline \hline$\mu_{i}$ & $-5 \%$ & 0 \\
\hline$\sigma_{i}$ & $20 \%$ & $20 \%$ \\
\hline$s_{0, i}$ & 36 & 40
\end{tabular}

$d=2, \quad N_{\max }=300, \quad n=25$

Out-of-the-money case

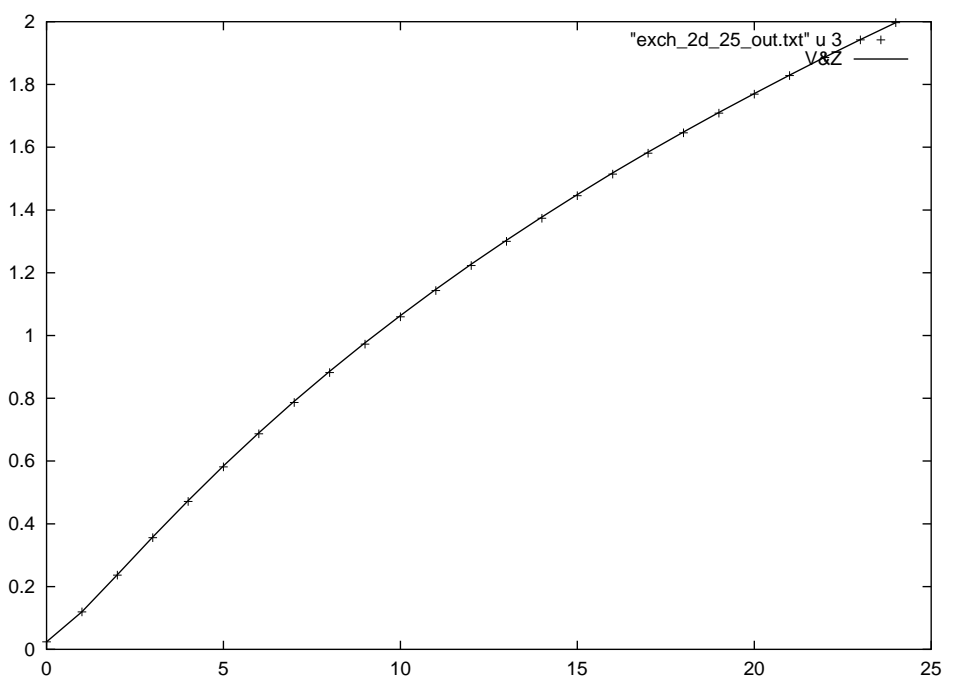

Figure 3: American exchange option price $\left(S_{1}-S_{2}\right)_{+}$fonction of the maturity where the number of layers is 25 and the number of points on the top is 300 . + depicts the price obtained with the method of quantization and - depicts the reference price (V \& Z) (cf. [41]).

$\mathrm{RR} \mathrm{n}^{\circ} 4465$ 
a)

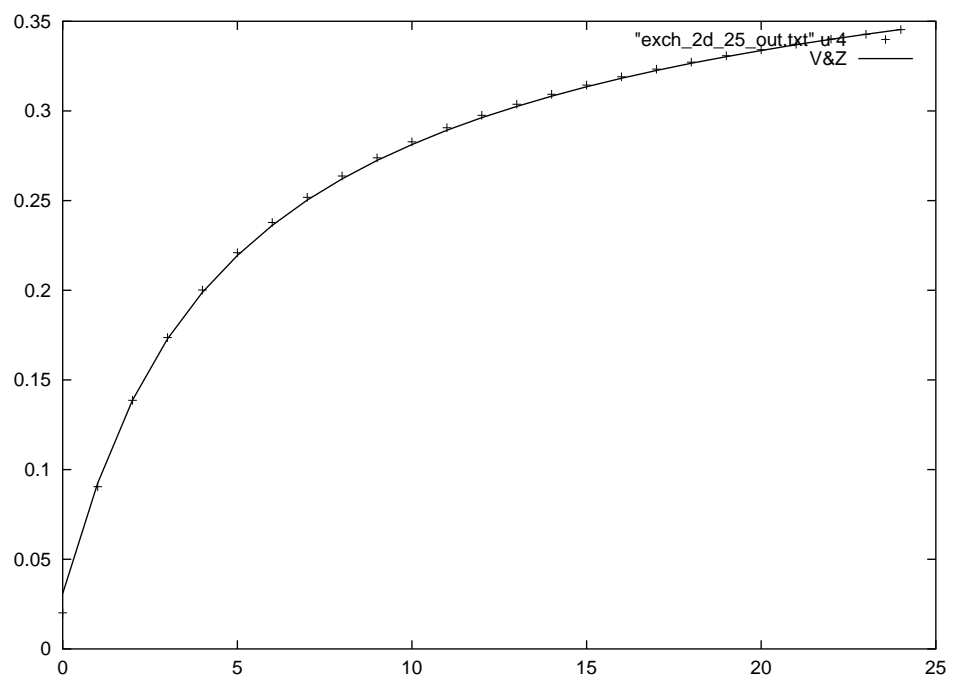

b)

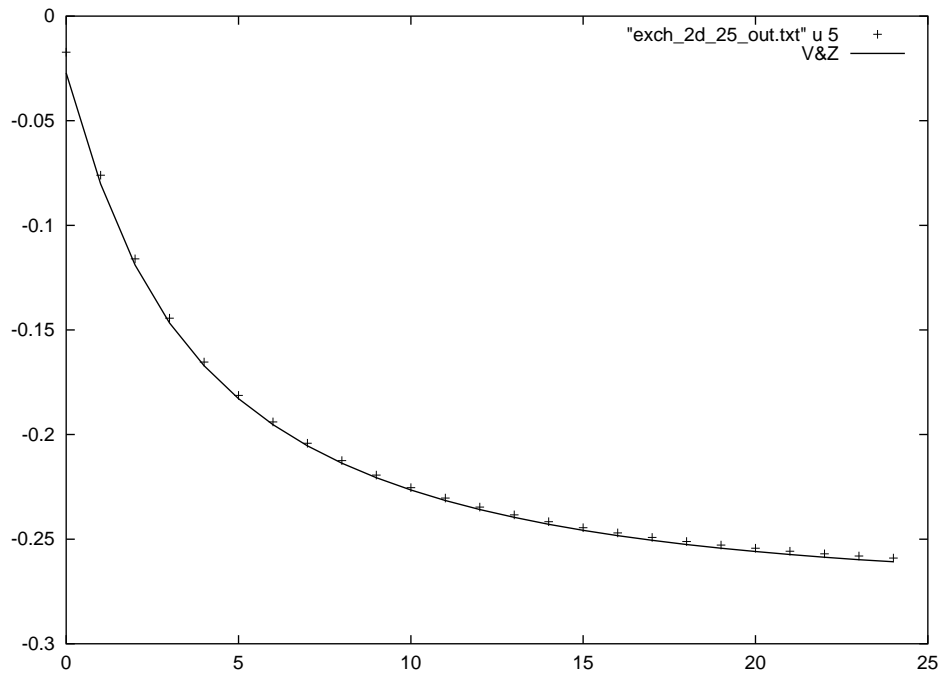

Figure 4: Hedging for the American option of Fig. 3. a) $\delta_{1}(+)$ fonction of the maturity. b) $\delta_{2}(+)$ fonction of the maturity. The reference price is denoted by - . 
Table 5: Value of the model parameter when $d=4$ in the in-the-money case.

\begin{tabular}{c||c|c|c|c}
$\mathrm{i}$ & 1 & 2 & 3 & 4 \\
\hline \hline$\mu_{i}$ & $-5 \%$ & 0 & 0 & 0 \\
\hline$\sigma_{i}$ & $14.14 \%$ & $14.14 \%$ & $14.14 \%$ & $14.14 \%$ \\
\hline$s_{0, i}$ & 6.32 & 6.32 & 6.00 & 6.00
\end{tabular}

$$
d=4, \quad N_{\max }=750, \quad n=20
$$

In-the-money case

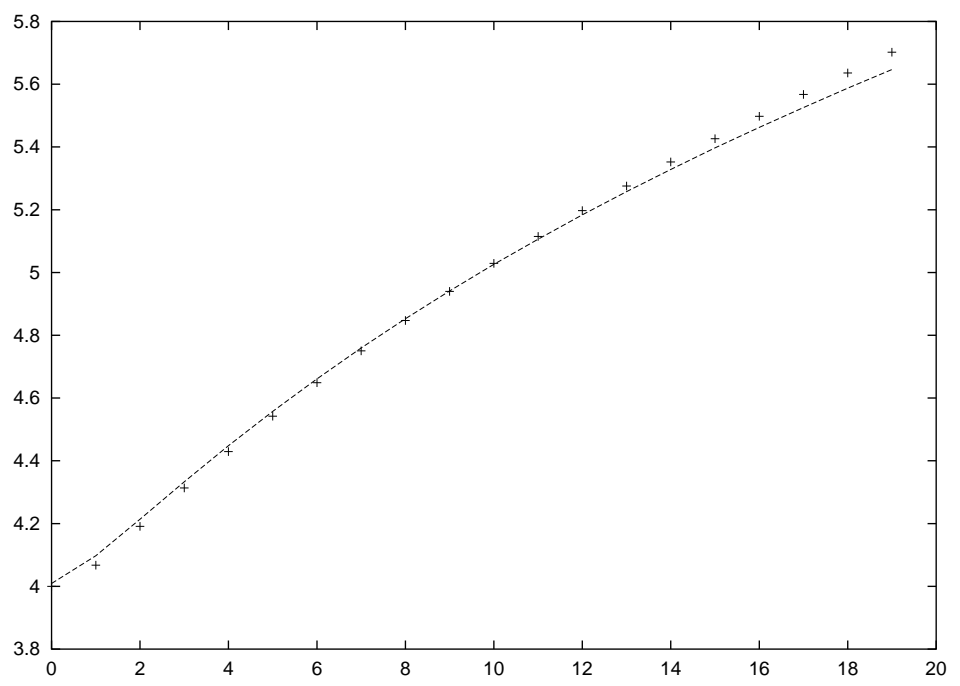

Figure 5: American exchange option price $\left(S_{1} S_{2}-S_{3} S_{4}\right)_{+}$in-the-money fonction of the maturity where the number of layers is 20 and the number of points on the top is 750 . + depicts the price obtained with the method of quantization and - depicts the reference price (V \& Z) (cf. [41]).

$\mathrm{RR} \mathrm{n}^{\circ} 4465$ 
a) $\delta_{1}(+), \delta_{2}(\times)$, Reference hedging $(-)$

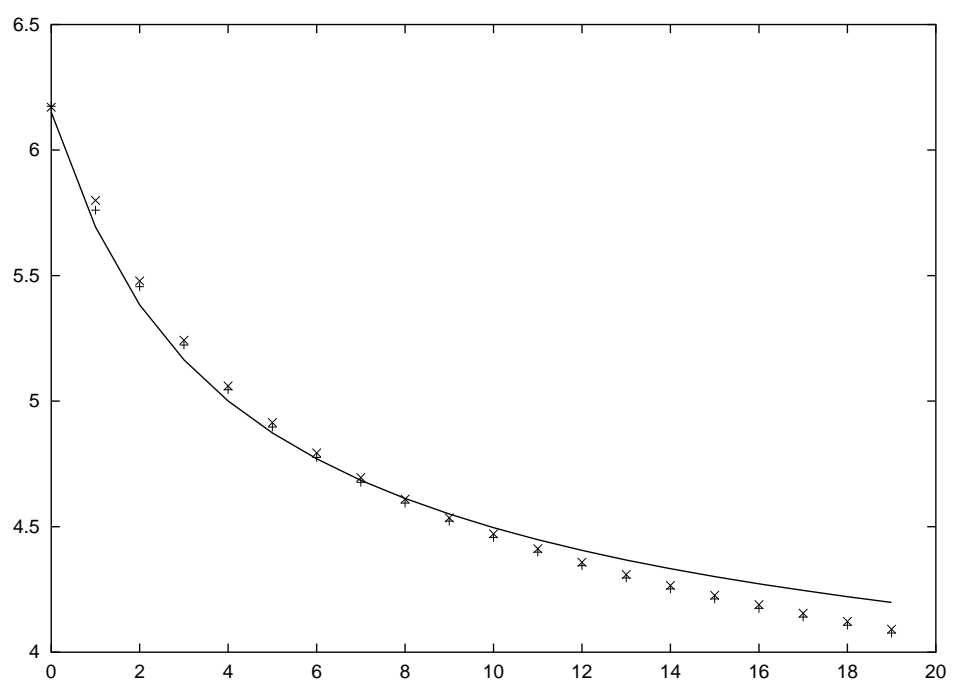

b) $\delta_{3}(+), \delta_{4}(\times)$, Reference hedging $(-)$

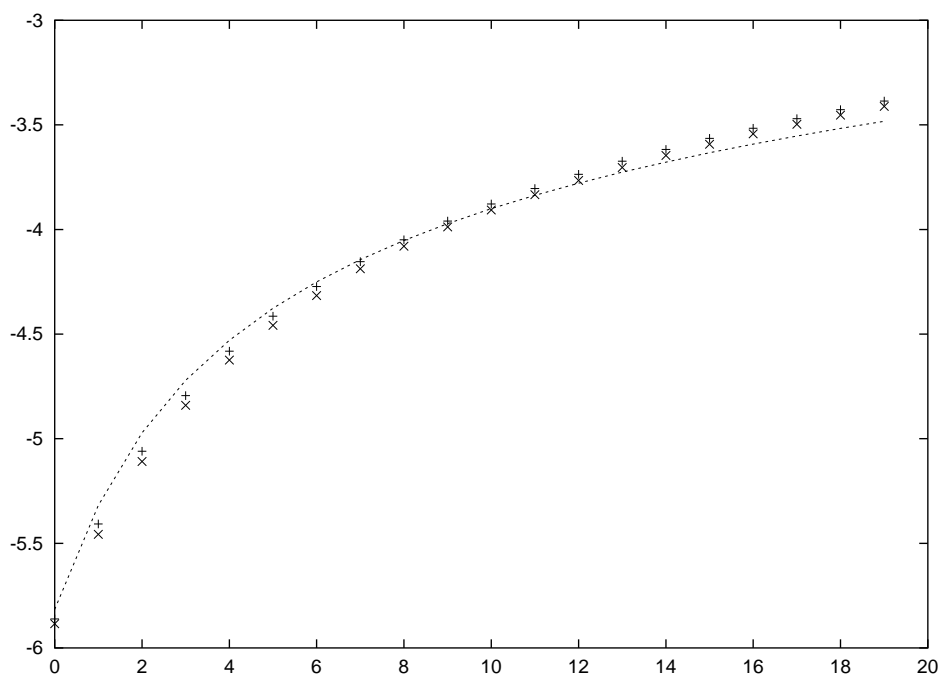

Figure 6: Hedging for the American option of Fig. 5. a) $\delta_{1}(+), \delta_{2}(\times)$ fonction of the maturity. b) $\delta_{3}(+), \delta_{4}(\times)$ fonction of the maturity. The reference price is denoted by -. 
Table 6: Value of the model parameter when $d=4$ in the out-of-the-money case.

\begin{tabular}{c||c|c|c|c}
$\mathrm{i}$ & 1 & 2 & 3 & 4 \\
\hline \hline$\mu_{i}$ & $-5 \%$ & 0 & 0 & 0 \\
\hline$\sigma_{i}$ & $14.14 \%$ & $14.14 \%$ & $14.14 \%$ & $14.14 \%$ \\
\hline$s_{0, i}$ & 6.00 & 6.00 & 6.32 & 6.32
\end{tabular}

$d=4, \quad N_{\max }=750, \quad n=20$

Out-of-the-money case

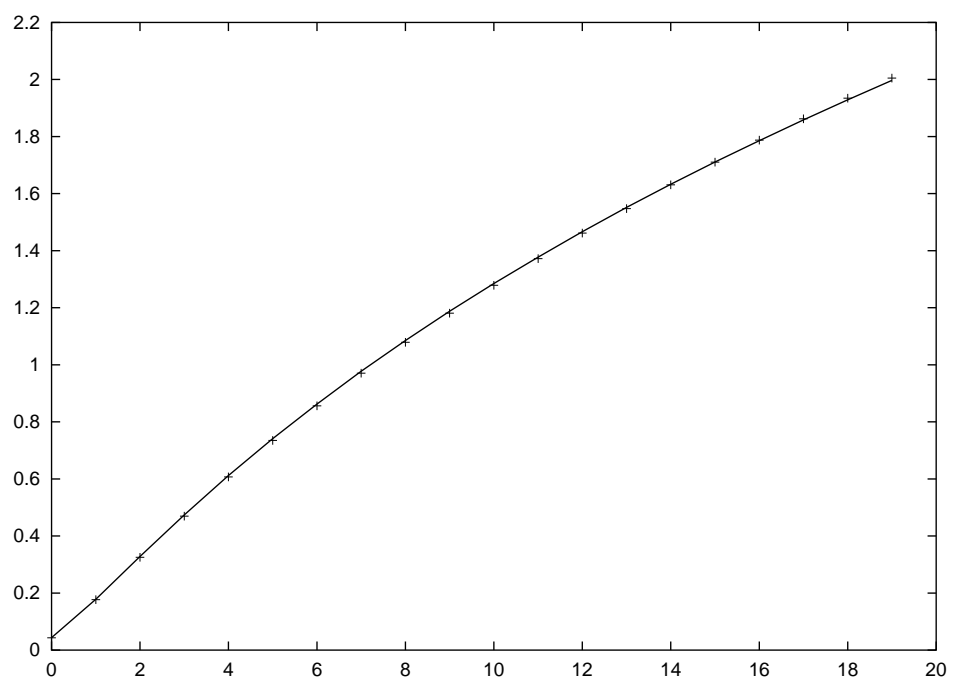

Figure 7: American exchange option price $\left(S_{1} S_{2}-S_{3} S_{4}\right)_{+}$out-the-money fonction of the maturity where the number of layers is 20 and the number of points on the top is 750 . + depicts the price obtained with the method of quantization and - depicts the reference price (V \& Z) (cf. [41]).

$\mathrm{RR} \mathrm{n}^{\circ} 4465$ 
a) $\delta_{1}(+), \delta_{2}(\times)$, Reference hedging $(-)$

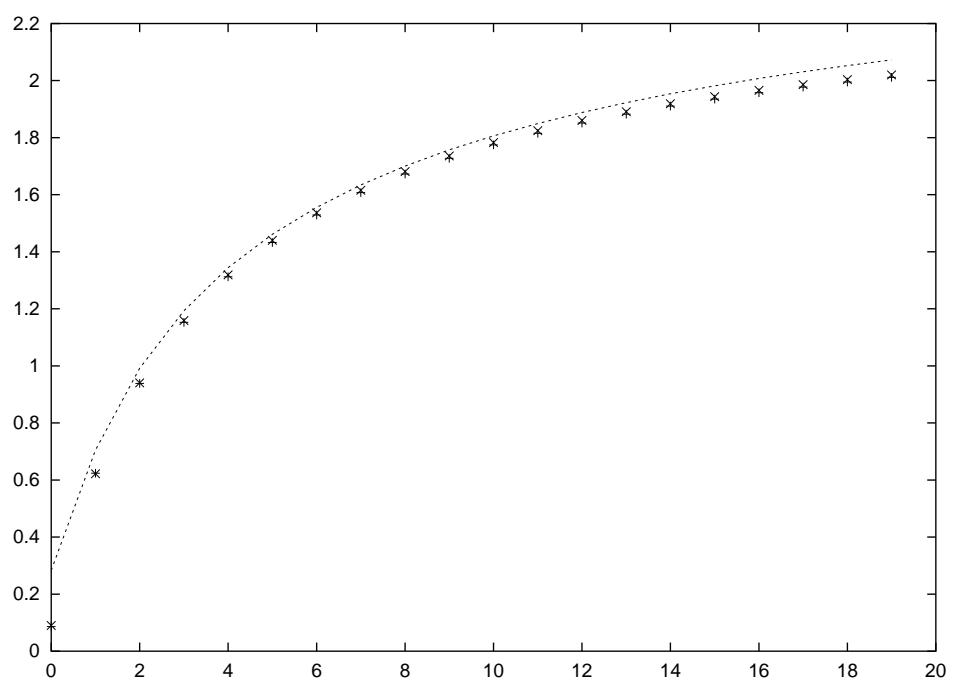

b) $\delta_{3}(+), \delta_{4}(\times)$, Reference hedging $(-)$

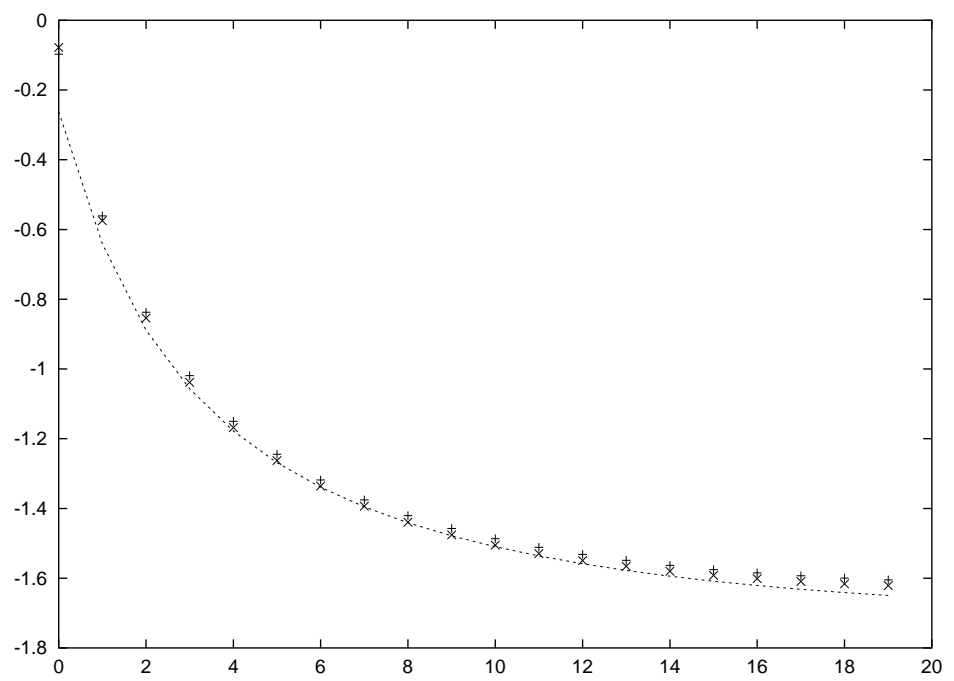

Figure 8: Hedging for the American option of Fig. 7. a) $\delta_{1}(+), \delta_{2}(\times)$ fonction of the maturity. b) $\delta_{3}(+), \delta_{4}(\times)$ fonction of the maturity. The reference price is denoted by -. 
Table 7: Value of the model parameter when $d=6$ in the in-the-money case.

\begin{tabular}{c||c|c|c|c|c|c}
$\mathrm{i}$ & 1 & 2 & 3 & 4 & 5 & 6 \\
\hline \hline$\mu_{i}$ & $-5 \%$ & 0 & 0 & 0 & 0 & 0 \\
\hline$\sigma_{i}$ & $11.55 \%$ & $11.55 \%$ & $11.55 \%$ & $11.55 \%$ & $11.55 \%$ & $11.55 \%$ \\
\hline$s_{0, i}$ & 3.42 & 3.42 & 3.42 & 3.30 & 3.30 & 3.30
\end{tabular}

$$
\begin{gathered}
d=6, \quad N_{\max }=1000, \quad n=25 \\
\text { In-the-money case }
\end{gathered}
$$

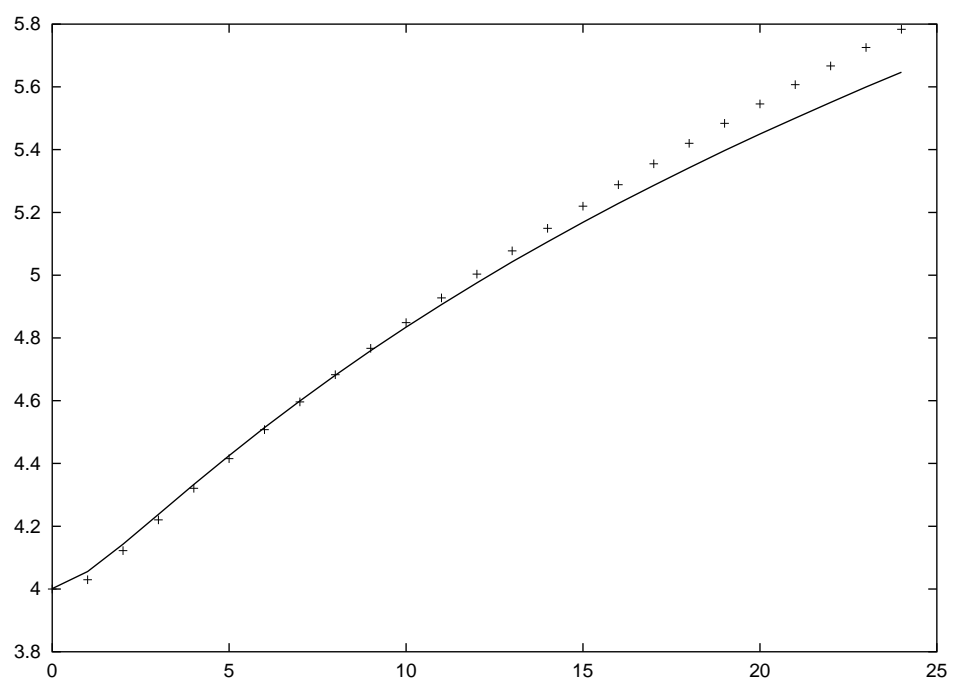

Figure 9: American exchange option price $\left(S_{1} S_{2} S_{3}-S_{3} S_{4} S_{5}\right)_{+}$fonction of the maturity where the number of layers is 25 and the number of points on the top is 1000 . + depicts the price obtained with the method of quantization and - depicts the reference price ( $\mathrm{V} \&$ Z) (cf. [41]). 
a)

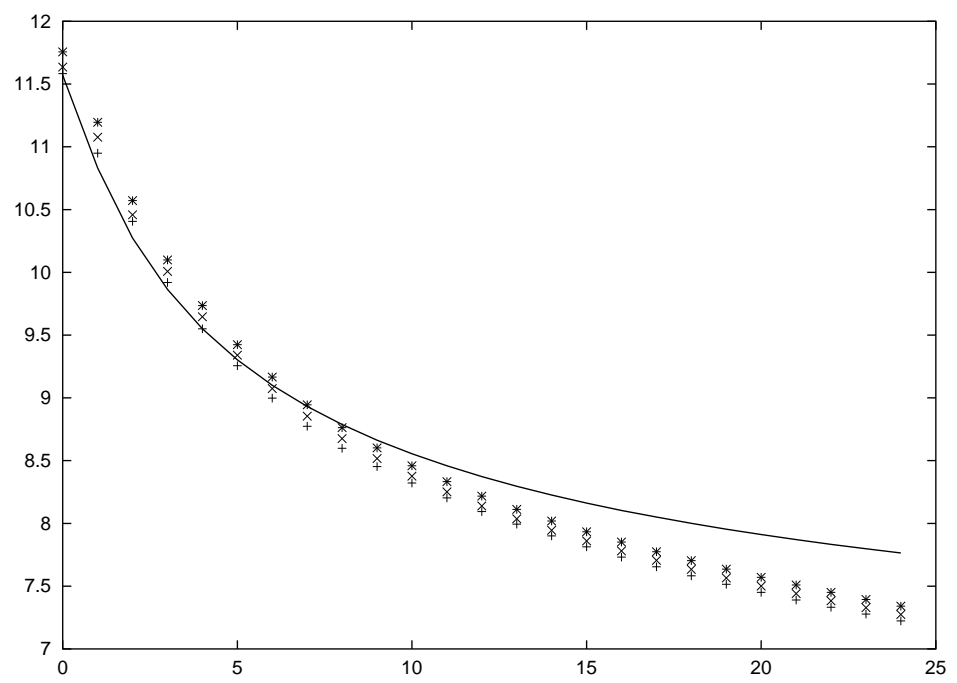

b)

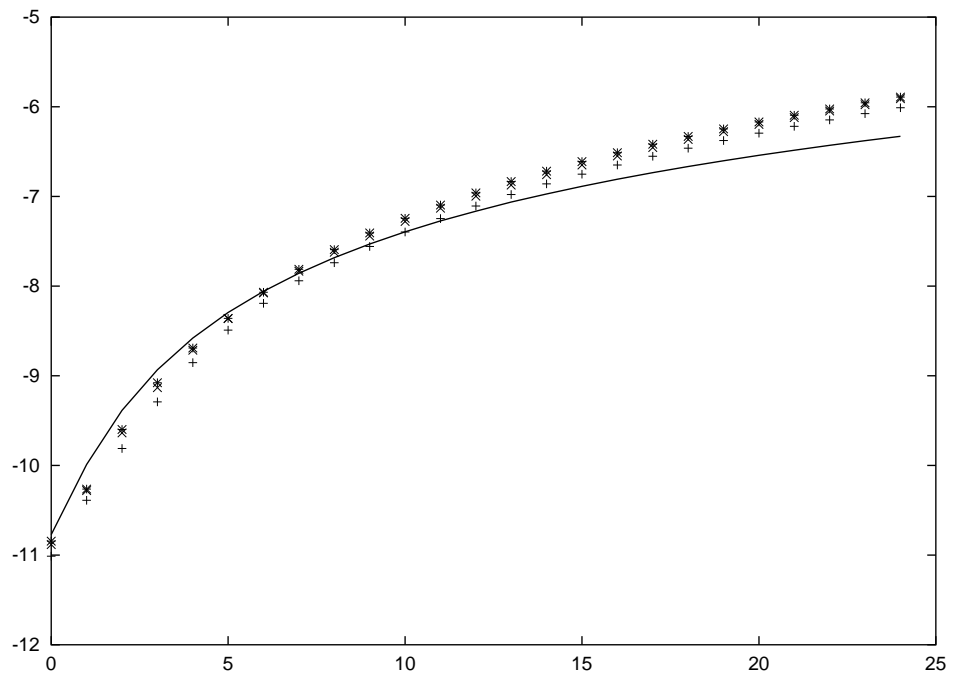

Figure 10: Hedging for the American option of Fig. 9. a) $\delta_{1}(+), \delta_{2}(\times), \delta_{3}(*)$ fonction of the maturity. b) $\delta_{4}(+), \delta_{5}(\times), \delta_{6}( \pm)$ fonction of the maturity. The reference price is denoted by - . 
Table 8: Value of the model parameter when $d=6$ in the out-of-the-money case.

\begin{tabular}{c||c|c|c|c|c|c}
$\mathrm{i}$ & 1 & 2 & 3 & 4 & 5 & 6 \\
\hline \hline$\mu_{i}$ & $-5 \%$ & 0 & 0 & 0 & 0 & 0 \\
\hline$\sigma_{i}$ & $11.55 \%$ & $11.55 \%$ & $11.55 \%$ & $11.55 \%$ & $11.55 \%$ & $11.55 \%$ \\
\hline$s_{0, i}$ & 3.30 & 3.30 & 3.30 & 3.42 & 3.42 & 3.42
\end{tabular}

$d=6, \quad N_{\max }=1000, \quad n=25$

Out-of-the-money case

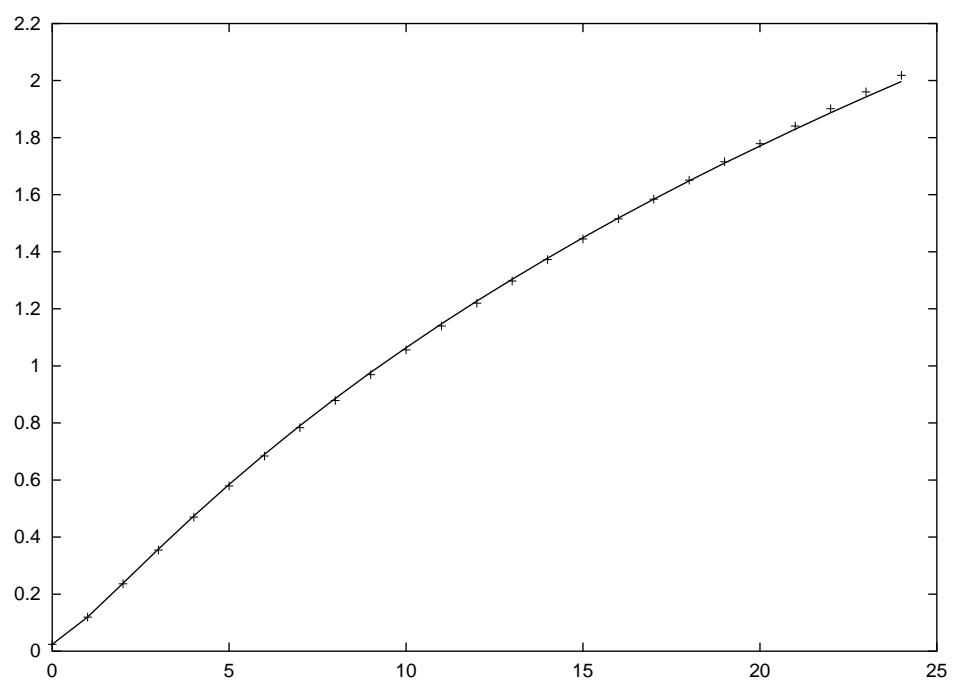

Figure 11: American exchange option price $\left(S_{1} S_{2} S_{3}-S_{3} S_{4} S_{5}\right)_{+}$fonction of the maturity where the number of layers is 25 and the number of points on the top is 1000 . + depicts the price obtained with the method of quantization and - depicts the reference price (V \& Z) (cf. [41]).

$\mathrm{RR} \mathrm{n}^{\circ} 4465$ 
a)

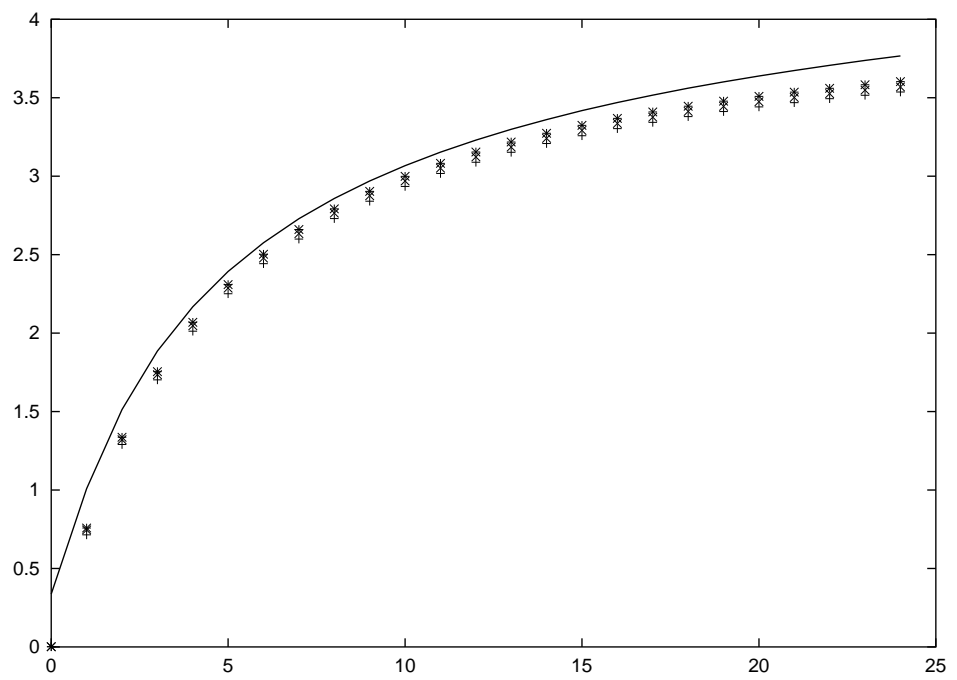

b)

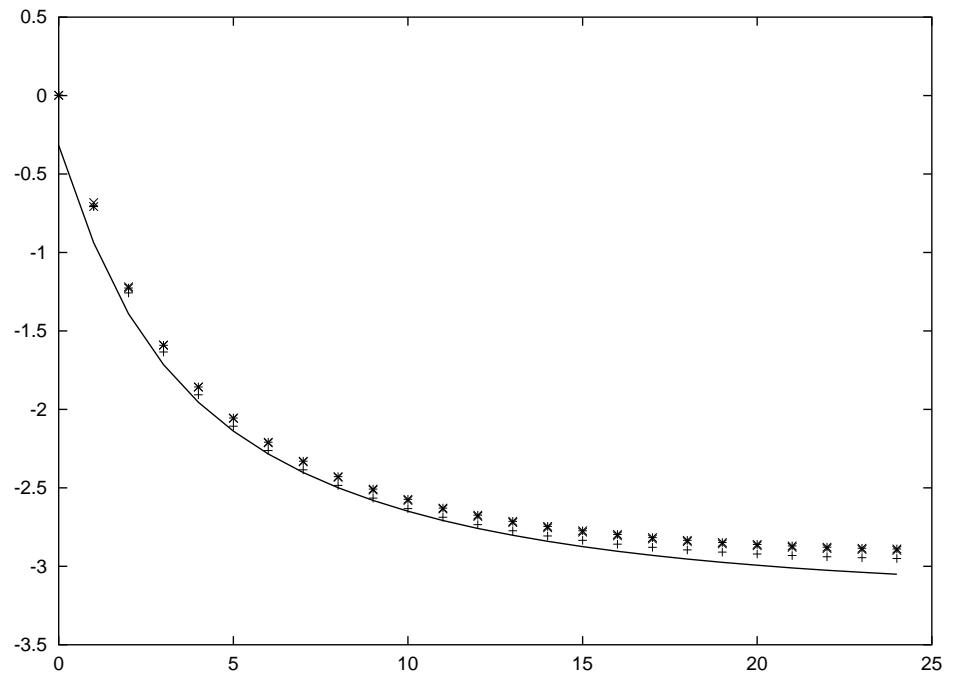

Figure 12: Hedging for the American option of Fig. 11. a) $\delta_{1}(+), \delta_{2}(\times), \delta_{3}(*)$ fonction of the maturity. b) $\delta_{4}(+), \delta_{5}(\times), \delta_{6}( \pm)$ fonction of the maturity. The reference price is denoted by - . 
Table 9: Value of the model parameter when $d=10$ in the in-the-money case.

\begin{tabular}{c||c|c|c|c|c|c|c|c|c|c}
$\mathrm{i}$ & 1 & 2 & 3 & 4 & 5 & 6 & 7 & 8 & 9 & 10 \\
\hline \hline$\mu_{i}$ & $-5 \%$ & 0 & 0 & 0 & 0 & 0 & 0 & 0 & 0 & 0 \\
\hline$\sigma_{i}$ & $8.94 \%$ & $8.94 \%$ & $8.94 \%$ & $8.94 \%$ & $8.94 \%$ & $8.94 \%$ & $8.94 \%$ & $8.94 \%$ & $8.94 \%$ & $8.94 \%$ \\
\hline$s_{0, i}$ & 2.09 & 2.09 & 2.09 & 2.09 & 2.09 & 2.04 & 2.04 & 2.04 & 2.04 & 2.04
\end{tabular}

$$
d=10, \quad N_{\max }=1000, \quad n=50
$$

In-the-money case

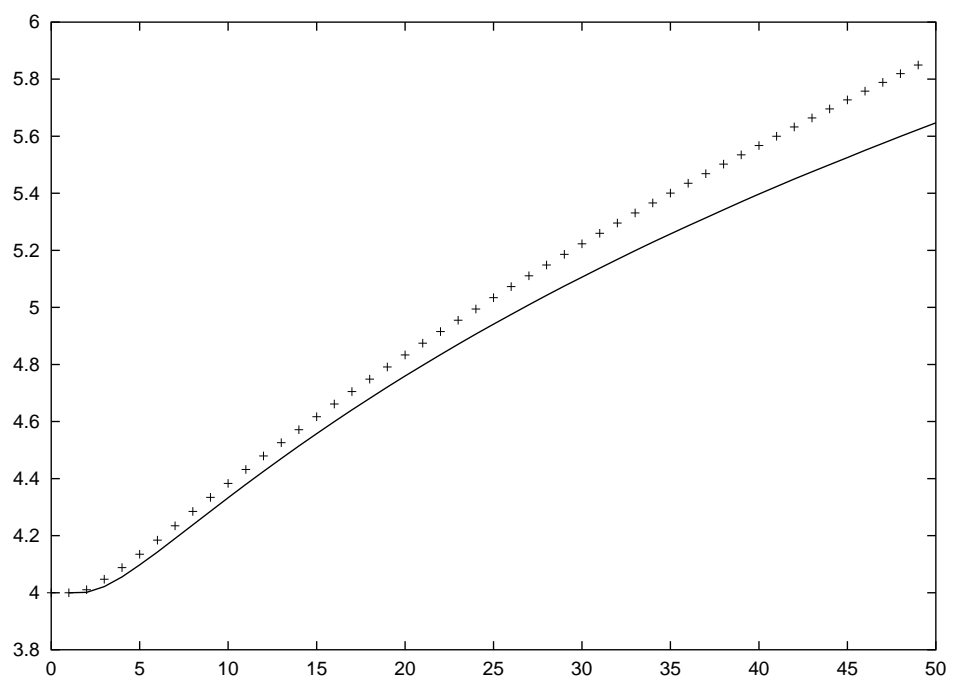

Figure 13: American exchange option price $\left(S_{1} S_{2} S_{3} S_{4} S_{5}-S_{6} S_{7} S_{8} S_{9} S_{10}\right)_{+}$fonction of the maturity where the number of layers is 50 and the number of points on the top is 1000 . + depicts the price obtained with the method of quantization and - depicts the reference price (V \& Z) (cf. [41]). 
a)

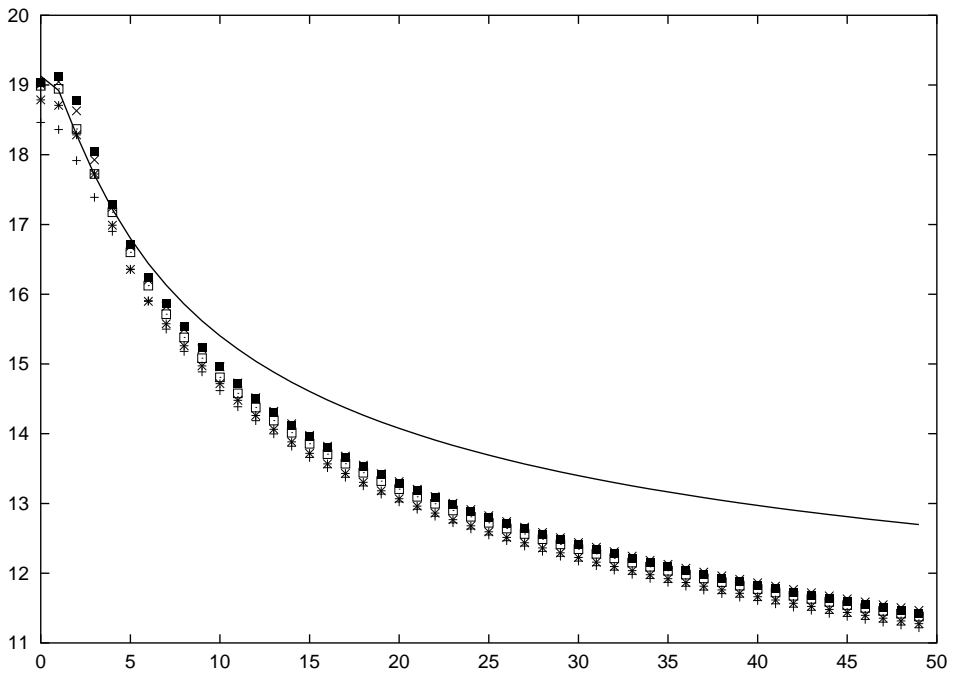

b)

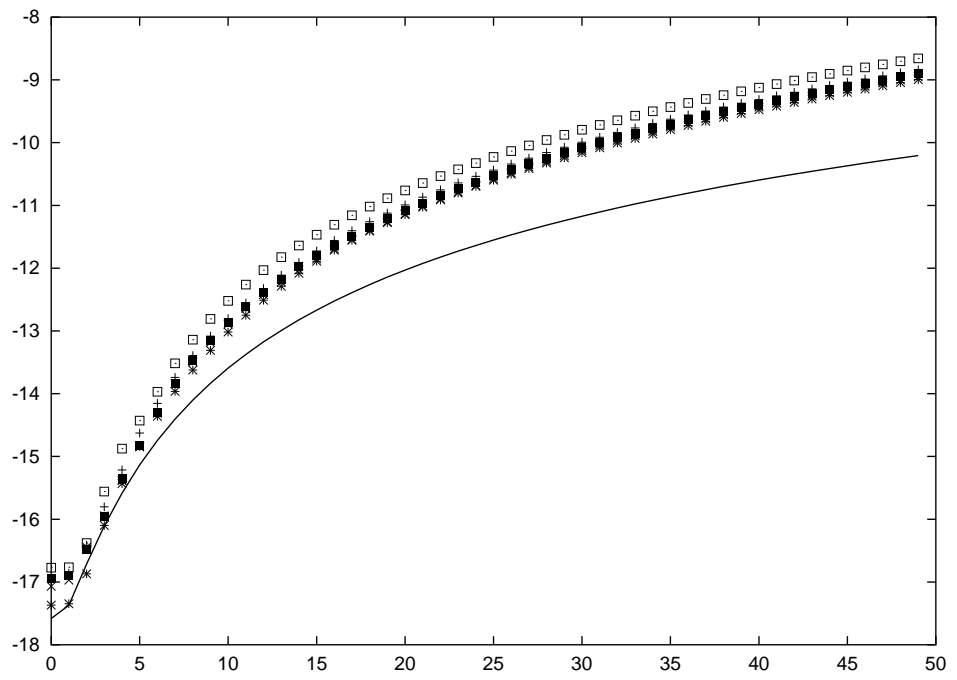

Figure 14: Hedging for the American option of Fig. 13. a) $\delta_{1}(+), \delta_{2}(\times), \delta_{3}(*), \delta_{4}(\mathbf{\square})$, $\delta_{5}(\square)$ fonction of the maturity. b) $\delta_{6}(+), \delta_{7}(\times), \delta_{8}(*), \delta_{9}(\boldsymbol{\square}), \delta_{10}(\square)$ fonction of the maturity. The reference price is denoted by - .

INRIA 
Table 10: Value of the model parameter when $d=10$ in the out-of-the-money case.

\begin{tabular}{c||c|c|c|c|c|c|c|c|c|c}
$\mathrm{i}$ & 1 & 2 & 3 & 4 & 5 & 6 & 7 & 8 & 9 & 10 \\
\hline \hline$\mu_{i}$ & $-5 \%$ & 0 & 0 & 0 & 0 & 0 & 0 & 0 & 0 & 0 \\
\hline$\sigma_{i}$ & $8.94 \%$ & $8.94 \%$ & $8.94 \%$ & $8.94 \%$ & $8.94 \%$ & $8.94 \%$ & $8.94 \%$ & $8.94 \%$ & $8.94 \%$ & $8.94 \%$ \\
\hline$s_{0, i}$ & 2.04 & 2.04 & 2.04 & 2.04 & 2.04 & 2.09 & 2.09 & 2.09 & 2.09 & 2.09 \\
& \\
$d=10, \quad N_{\max }=1000, \quad n=50$
\end{tabular}

Out-of-the-money case

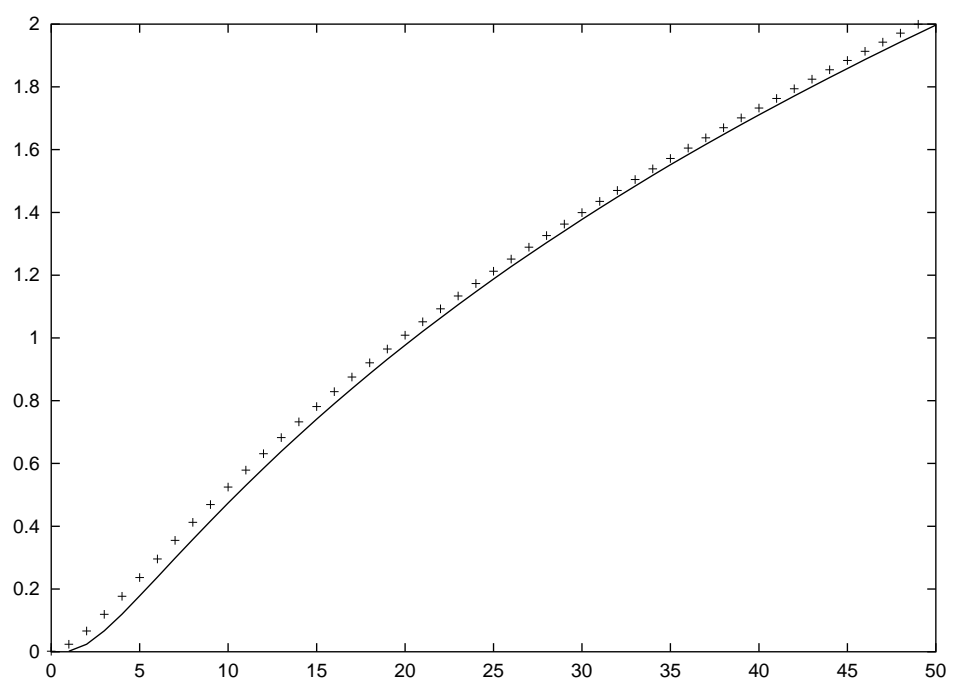

Figure 15: American exchange option price $\left(S_{1} S_{2} S_{3} S_{4} S_{5}-S_{6} S_{7} S_{8} S_{9} S_{10}\right)_{+}$fonction of the maturity where the number of layers is 50 and the number of points on the top is 1000 . + depicts the price obtained with the method of quantization and - depicts the reference price (V \& Z) (cf. [41]). 
a)

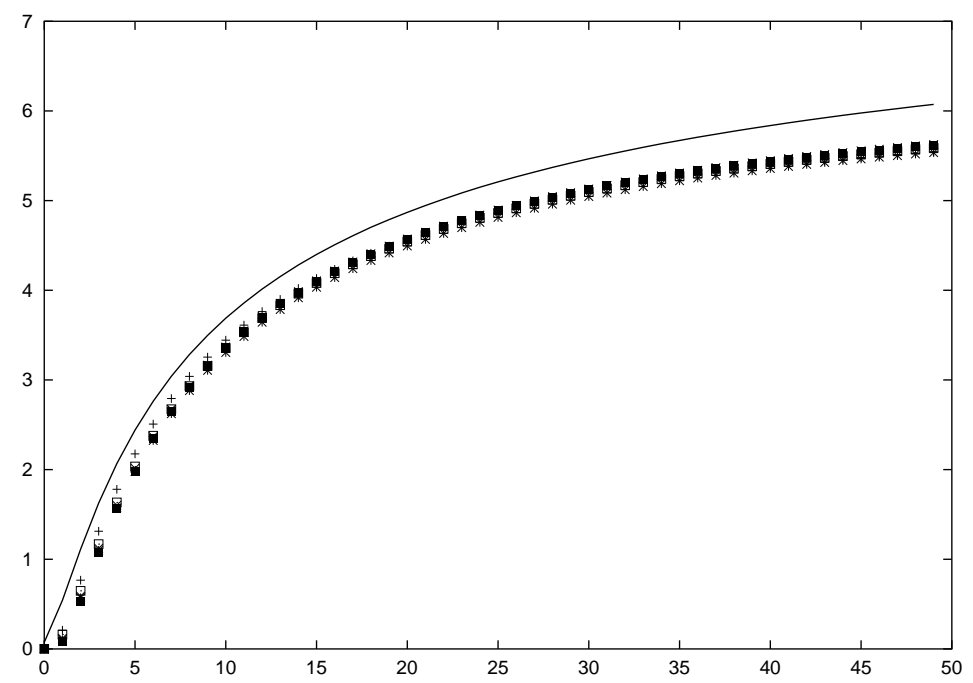

b)

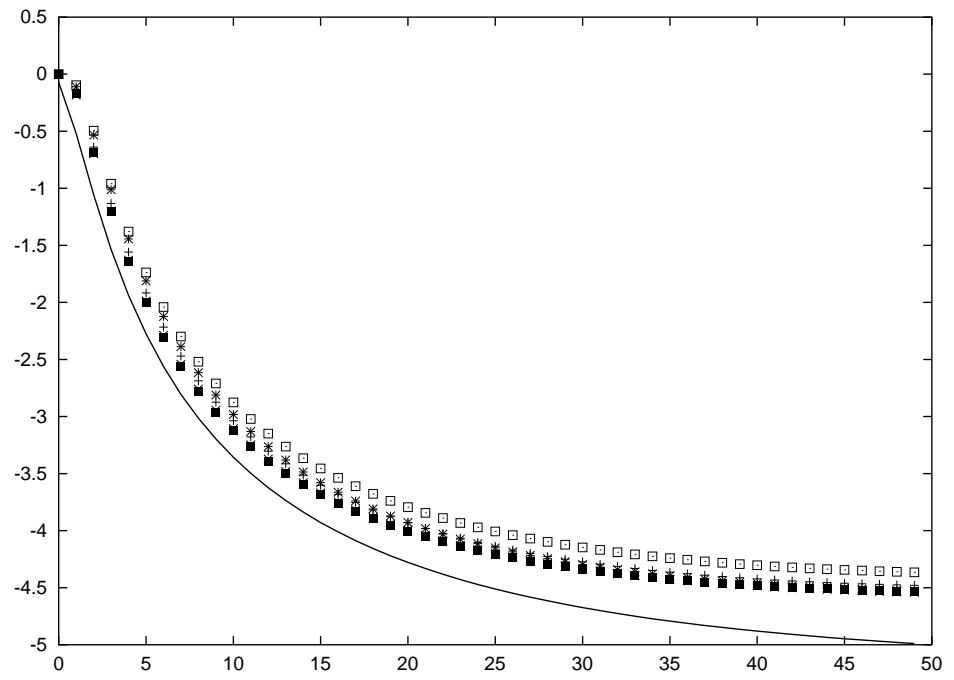

Figure 16: Hedging for the American option of Fig. 15. a) $\delta_{1}(+), \delta_{2}(\times), \delta_{3}(*), \delta_{4}(\boldsymbol{\square})$, $\delta_{5}(\square)$ fonction of the maturity. b) $\delta_{6}(+), \delta_{7}(\times), \delta_{8}(*), \delta_{9}(\boldsymbol{\square}), \delta_{10}(\square)$ fonction of the maturity. The reference price is denoted by - . 


\section{References}

[1] D.G. Aronson, Bounds for the fundamental solution of a parabolic equation, Bull. of the A.M.S., 73, 1967, pp.890-903.

[2] V. Bally, M.E. Caballero, B. Fernandez, N. El Karoui, Reflected BSDE's, PDE's and Variational inequalities. Pré-print INRIA, 2002.

[3] V. Bally, D. Talay, The law of the Euler scheme for stochastic differential equations. I: Convergence rate of the distribution function, Probab. Theory Relat. Fields, 104, No.1, pp.43-60, 1996.

[4] V. Bally, G. Pagès, A quantization algorithm for solving multi-dimensional Optimal Stopping problems, technical report $\mathrm{n}^{\circ} 628$, Laboratoire de Probabilités \& modèles aléatoires, Université Paris VI (France), 2000.

[5] V. Bally, G. Pagès, J. Printems, A stochastic quantization method for non linear problems, Monte Carlo Methods and Applications, 7, $\mathrm{n}^{0} 1-2$, pp.21-34, 2001.

[6] V. Bally, G. Pagès, J. Printems, First order schemes in the numerical quantization method, forthcoming in (selected papers of the colloquium Application of Malliavin calculus to Finance).

[7] V. Bally, G. Pagès, Error analysis of a quantization algorithm for obstacle problems, forthcoming in Stoch. Proc. and their Appl., 2002.

[8] J. Barraquand, D. Martineau, Numerical valuation of high dimensional multivariate American securities, Journal of Finance and Quantitative Analysis, 30, 1995.

[9] A. Bensousan, J.L.Lions, Applications of the Variational Inequalities in Stochastic Control, North Holland, 1982, or Applications des inéquations variationnelles en contrôle stochastique, Dunod, Paris, 1978.

[10] N. Bouleau, D. Lamberton, Residual risks and hedging strategies in Markovian markets, Stoch. Proc. and their Appl., 33, pp.131-150, 1989.

[11] M. Broadie, P. Glasserman, Pricing American-Style Securities Using Simulation, Journal of Economic Dynamics and Control, 21, $n^{0} 8-9$, pp.1323-1352, 1997.

[12] J. Bucklew, G. Wise, Multidimensional Asymptotic Quantization Theory with $r^{\text {th }}$ Power distortion Measures, IEEE on Information Theory, Special issue on Quantization, 28, $\mathrm{n}^{0}$ 2, pp.239-247, 1982.

[13] D. Chevance, Thèse de l'Université de Provence \& INRIA, Septembre 1997, 140p.

[14] D. Chevance, Numerical methods for backward stochastic differential equations, Numerical Methods in Finance, L. Rogers and D. Talay eds., Publications of the Newton Institute series, Cambridge University Press, 1997.

[15] P. Cohort, A geometric approach for the uniqueness of a locally optimal quantizer, Technical Report, Labo. Probabilités, Univ. Paris 6 (France), 1997, submitted for publication.

[16] P. Cohort, Strong Law of large Number for the random distortion, Technical Report, Centre de Mathématiques de l'Université Paris 12, submitted for publication.

[17] P. Cohort, Sur quelques problèmes de quantification, Thèse de l'Université Pierre et Marie Curie, Janvier 2000.

$\mathrm{RR} \mathrm{n}^{\circ} 4465$ 
a) Residual risk in $d=4$.

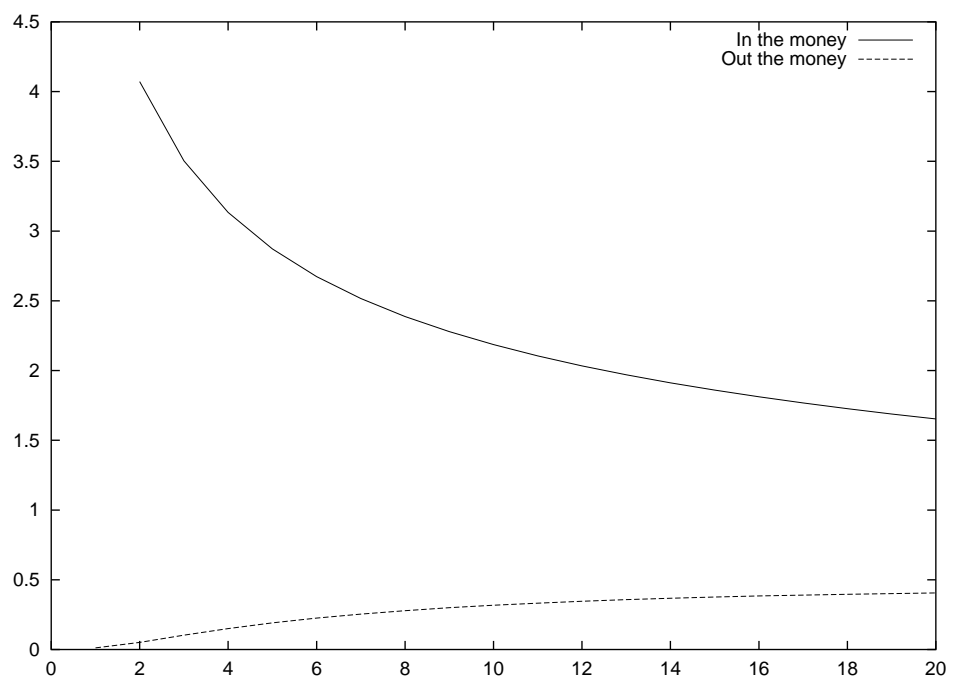

b) Residual risk in $d=6$.

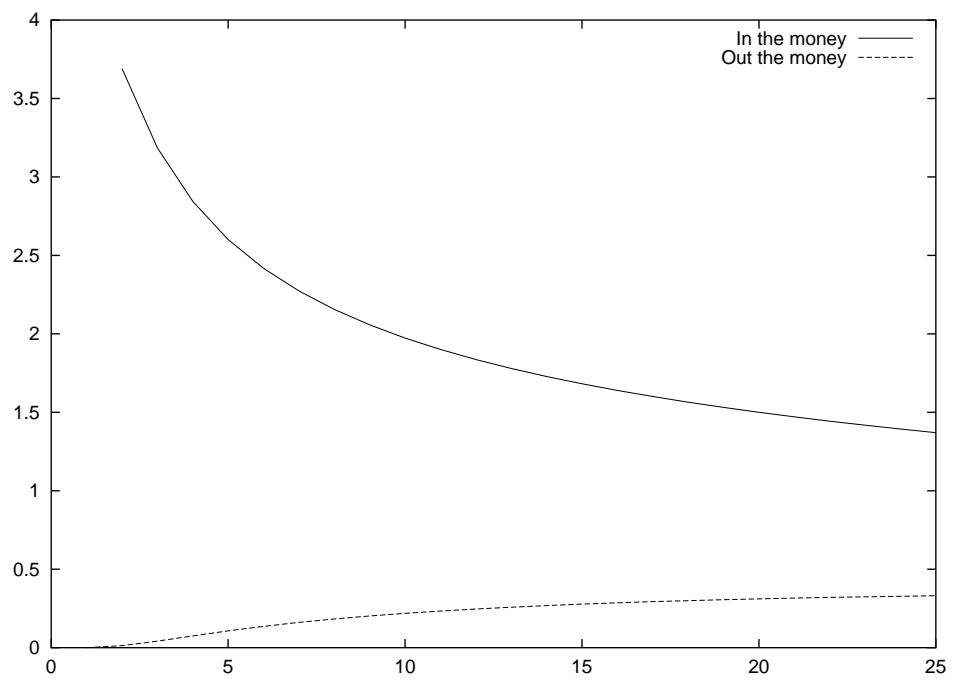

c)

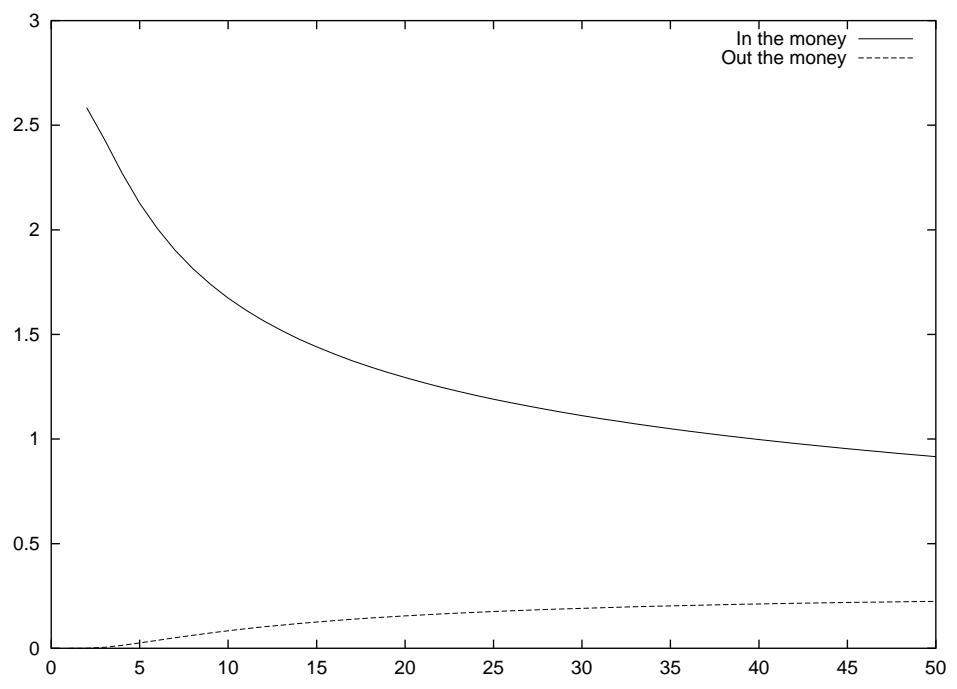

INRIA 
[18] A.P. Caverhill, N. Webber, American options: theory and numerical analysis, in Options: recent advances in theory and practise, Manchester University press, 1990.

[19] M. Duflo, Random Iterative systems, Berlin, Springer, 1998.

[20] N. El Karoui, C. Kapoudjan, E. Pardoux, S. Peng, M.C. Quenez, Reflected solutions of Backward Stochastic Differential Equations and related obstacle problems for PDE's, The Ann. of Proba., 25, No 2, pp.702-737, 1997.

[21] H. Föllmer, D. Sondermann, Hedging of non redundant contingent claims, Contributions to Mathematical Economics, pp.205-223, North-Holland, Amsterdam, 1986.

[22] É. Fournié, J.M. Lasry, J. Lebouchoux, P.L. Lions, N. Touzi, Aplications of Malliavin calculus to Monte Carlo methods in Finance, Finance \&s Stochastics, 3, pp.391-412, 1999.

[23] É. Fournié, J.M. Lasry, J. Lebouchoux, P.L. Lions, Aplications of Malliavin calculus to Monte Carlo methods in Finance II, Finance \&S Stochastics, 5, pp.201-236, 2001.

[24] A. Friedmann, Partial Differential Equations and Applications, Academic Press, 1, 1975.

[25] S. Graf, H. Luschgy, Foundations of quantization for random vectors, Lecture Notes in Mathematics $\mathrm{n}^{0} 1730$, Springer, 2000, 230p.

[26] A. Gersho, R. Gray (eds.), IEEE on Information Theory, Special issue on Quantization, 28, 1982.

[27] N. Ikeda and S. Watanabe Stochastic Differential Equations and Diffusion processes, second edition, North Holland 1989.

[28] Kushner H.J., Approximation and weak convergence methods for random processes, with applications to stochastic systems theory, MIT Press Series in Signal Processing, Optimization, and Control, 6, MIT Press, Cambridge, MA, (1977), 1984, 269 pp.

[29] Kushner H.J., Dupuis P., Numerical methods for stochastic control problems in continuous time, $2^{\text {nd }}$ edition, Applications of Mathematics, 24, Stochastic Modelling and Applied Probability, Springer-Verlag, New York, 2001, 475 pp.

[30] H.J. Kushner, G.G. Yin, Stochastic Approximations Algorithms and Applications, Springer, New York, 1997.

[31] S. Kusuoka and D. Stroock, Application of the Malliavin calculus II, J. Fac. Sci. Univ. Tokyo, Sect IA Math., 32, pp.1-76, 1985.

[32] D. Lamberton, B. Lapeyre, Introduction to stochastic calculus applied to Finance, Chapman \& Hall, London, 1996, 185 pp.

[33] D. Lamberton, Brownian optimal stopping and random walks, technical report Univ. Marnela-Vallée, 1998 (revised 1999).

[34] D. Lamberton D., B. Lapeyre, Introduction to Stochastic Calculus applied to Finance, Chapman \& Hall, 1996, 185p.

[35] P.L. Lions, H. Régnier, Calcul des prix et des sensibilités d'une option américaine par une méthode de Monte Carlo, working paper.

[36] F.A. Longstaff, E.S. Schwartz, Valuing American options by simulation: a simple least-squares approach, Review of Financial Studies, 14, 113-148, 2001.

[37] J. Neveu, Martingales à temps discret, Masson, Paris, 1971, 215p.

$\mathrm{RR} \mathrm{n}^{\circ} 4465$ 
[38] G. Pagès, A space vector quantization method for numerical integration, Journal of Applied and Computational Mathematics, 89, pp.1-38, 1997.

[39] D. Revuz, M. Yor, Continuous Martingales and Brownian Motion, Springer-Verlag, $2^{\text {nd }}$ edition, Berlin-Heidelberg, 1991, 560p.

[40] J.N. Tsitsiklis, B. Van Roy, Optimal stopping of Markov processes: Hilbert space theory, approximation algorithms, and an application to pricing high-dimensional financial derivatives, IEEE Trans. Automat. Control, 44, $\mathrm{n}^{0} 10,1999$, pp.1840-1851.

[41] S. Villeneuve, A. Zanette (2002), Parabolic A.D.I. methods for pricing american option on two stocks, forthcoming in Mathematics of Operation Research. 


\section{ANNEX}

Proposition 4 (a) If b, $c \in \mathcal{C}_{b}^{\infty}\left(\mathbb{R}^{d}\right)$ and $c$ is uniformly elliptic, then both $\left(S_{t_{k}}\right)_{0 \leq k \leq n}$ and $\left(\bar{S}_{t_{k}}\right)_{0 \leq k \leq n}$ satisfy the domination property (31) with

$$
\varphi_{k}:=c_{b, \sigma} \sqrt{t_{k}} \quad\left(c_{b, \sigma}>0\right) \quad \text { and } \quad R:=Z \sim \mathcal{N}\left(0 ; I_{d}\right) .
$$

(b) In the extended Black \& Scholes model (1), if $\sigma \in \mathcal{C}_{b}^{\infty}\left(\mathbb{R}^{d}\right)$ is uniformly elliptic, then both $\left(S_{t_{k}}\right)_{0 \leq k \leq n}$ and $\left(\bar{S}_{t_{k}}\right)_{0 \leq k \leq n}$ satisfy the domination property (31) with

$$
\varphi_{k}:=c_{b, \sigma} \sqrt{\frac{t_{k}}{T}} \quad\left(c_{b, \sigma}>0\right) \quad \text { and } \quad R:=\left(s_{0}^{i} \psi\left(\sqrt{T} Z^{i}\right)\right)_{1 \leq i \leq d}, Z \sim \mathcal{N}\left(0 ; I_{d}\right),
$$

where $\psi(u):=\left(u^{i}+e^{u^{i}}\right)_{1 \leq i \leq d}, u=\left(u^{1}, \ldots, u^{d}\right) \in \mathbb{R}^{d}$.

Proof: (a) cf. [4], Theorem 4.

(b) One starts from the obvious inequality, valid for every $u, v \in \mathbb{R}$ and every $\rho>0$,

$$
\left|e^{\rho v}-e^{\rho u}\right| \leq \rho\left|v+e^{v}-\left(u+e^{u}\right)\right|
$$

The diffusion $Y_{t}:=\ln S_{t}$ starting at 0 is clearly a diffusion with diffusion coefficient $\sigma\left(S_{t}\right)$, hence $\ln S_{t}$ is uniformly elliptic. It follows from item $(a)$ that the density function $\pi_{\ln S_{t_{k}}}$ satisfies

$$
\pi_{\ln S_{t_{k}}}(y) \leq \alpha \pi_{\sqrt{\beta t_{k}} Z}(y), \quad(\alpha, \beta>0) .
$$

Consequently, if $X_{k}:=S_{t_{k}}$ starting now at $X_{0}:=s_{0}>0$, one has for every $N$-tuple $x \in\left(\mathbb{R}_{+}^{d}\right)^{N}$

$$
D_{N}^{X_{k}, p}(x)=\mathbb{E}\left(\min _{1 \leq i \leq N}\left|\left(s_{0}^{\ell} e^{Y_{t_{k}}^{\ell}}\right)_{1 \leq \ell \leq d}-x_{i}\right|^{p}\right) \leq \alpha \mathbb{E}\left(\min _{1 \leq i \leq N}\left|\left(s_{0}^{\ell} e^{\beta t_{k} Z_{t_{k}}^{\ell}}\right)_{1 \leq \ell \leq d}-x_{i}\right|^{p}\right) .
$$

Now, one easily derives (with obvious notations) that

$$
\underline{D}_{N}^{X_{k}, p} \leq \alpha \inf _{y \in\left(\mathbb{R}^{d}\right)^{N}} \mathbb{E}\left(\min _{1 \leq i \leq N}\left|\left(s_{0}^{\ell}\left(e^{\beta t_{k} Z_{t_{k}}^{\ell}}-e^{\beta t_{k} y_{i}^{\ell}}\right)\right)_{1 \leq \ell \leq d}\right|^{p}\right) .
$$

For every $i \in\{1, \ldots, n\}$, Inequality (84) yields

$$
\sum_{\ell=1}^{d}\left(s_{0}^{\ell}\right)^{2}\left(e^{\sqrt{\beta t_{k}} Z^{\ell}}-e^{\sqrt{\beta t_{k}} y_{i}^{\ell}}\right)^{2} \leq\left(\sqrt{\frac{t_{k}}{T}}\right)^{p} \sum_{\ell=1}^{d}\left(s_{0}^{\ell}\right)^{2}\left(\psi\left(\sqrt{T} Z^{\ell}\right)-\psi\left(\sqrt{T} y_{i}^{\ell}\right)\right)^{2}
$$

which finally yields the expected result since $u \mapsto s_{0}^{\ell} \psi(\sqrt{T} u)$ is a bijective from $\mathbb{R}$ onto $\mathbb{R}_{+}^{*}$. $\diamond$ 


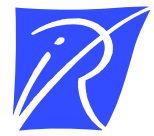

Unité de recherche INRIA Rocquencourt Domaine de Voluceau - Rocquencourt - BP 105 - 78153 Le Chesnay Cedex (France)

Unité de recherche INRIA Lorraine : LORIA, Technopðle de Nancy-Brabois - Campus scientifique 615, rue du Jardin Botanique - BP 101 - 54602 Villers-lès-Nancy Cedex (France)

Unité de recherche INRIA Rennes : IRISA, Campus universitaire de Beaulieu - 35042 Rennes Cedex (France)

Unité de recherche INRIA RhOne-Alpes : 655, avenue de 1'Europe - 38330 Montbonnot-St-Martin (France)

Unité de recherche INRIA Sophia Antipolis : 2004, route des Lucioles - BP 93 - 06902 Sophia Antipolis Cedex (France)

INRIA - Domaine de Voluceau - Rocquencourt, BP 105 - 78153 Le Chesnay Cedex (France)

http://www.inria.fr

ISSN 0249-6399 